Running Head: NETWORK APPROACH TO REPUTATIONS

Perceiving Personality Through the Grapevine: A Network Approach to Reputations

Cory K. Costello \& Sanjay Srivastava

University of Oregon

Author Note

Cory K. Costello and Sanjay Srivastava, University of Oregon, Department of Psychology, 1227 University of Oregon, Eugene, OR, 97403.

Correspondence concerning this article should be addressed to Cory K. Costello, who is now at the Department of Psychology, University of Michigan, 530 Church Street, University of Michigan, Ann Arbor, MI 48109, email: costck@umich.edu

Final version accepted for publication. The previous version referenced in the text can be found at https://psyarxiv.com/8wg25/download/?version=2.

CAmerican Psychological Association, 2020. This paper is not the copy of record and may not exactly replicate the authoritative document published in the APA journal. Please do not copy or cite without author's permission. The final article is available, upon publication, at: $10.1037 /$ pspp0000362. 
NETWORK APPROACH TO REPUTATIONS

\author{
Abstract \\ Reputations are critical in human social life: they allow people to share and act on information \\ about one another, even when they have never met. Reputations can be conceptualized as \\ information about a target person that is stored in networks of perceivers and transmitted through \\ either direct interaction or hearsay. We present a novel paradigm that integrates the network \\ approach with interpersonal perception research. We apply that paradigm to study the consensus, \\ accuracy, positivity bias, and consequences of personality trait information in hearsay-based \\ reputations. In two preregistered studies $(N=260$ and 369), we created naturalistic \\ micronetworks in the lab in which participants interacted and got to know one another, then later \\ described each other to naïve third parties. Across studies, we use the extended Social Accuracy \\ Model (Wessels et al., 2020) and an extension of the domain-wise correlational approach \\ (Kenny, 1994). Hearsay-based reputations as positively biased as direct reputations. They \\ showed strong consensus (agreement) with direct reputations and modest accuracy, suggesting \\ that they can consolidate around an inaccurate representation. Perceivers' extraversion was \\ associated with more biased hearsay reputations. Experimentally manipulating the context of the \\ hearsay exchange had no detectable impact on hearsay consensus or accuracy. Hearsay \\ reputations were consequential, affecting the extent to which perceivers thought targets would be \\ good leaders or friends. These results provide initial insights into reputation networks and \\ suggest several important future directions for the network approach to reputations. We also \\ present open materials and data analysis software for others to extend the reputations-as- \\ networks approach. \\ Keywords: Reputation; Personality; Interpersonal Perception; Gossip; Social Networks
}


NETWORK APPROACH TO REPUTATIONS

\section{Perceiving through the Grapevine: A Network Approach to Reputations}

Familiar phrases like "your reputation precedes you" capture a simple truth about our social world: reputations extend beyond people who have met one another, communicated further through the processes of gossip and hearsay (Craik, 2009). Sharing reputational knowledge is thought to at least partly underpin the unprecedented scale and cooperativeness of human social groups, by both expanding the volume of our social knowledge and allowing communities to act on shared knowledge about an individual (Dunbar, 2004; Feinberg, Willer, \& Schultz, 2014; Wu et al., 2015). Indeed, the desire and ability to share reputational knowledge is likely one factor behind the rich personality lexicons found in natural languages, which have served as a databases for identifying structural models of personality like the Big 5 and Big 6 (Saucier \& Goldberg, 1996; Saucier \& Srivastava, 2015; Wood, 2015). Some theories even suggest that trait terms are primarily for describing others (Buss \& Craik, 1983; Hogan, 1994; Srivastava, 2010; Saucier \& Srivastava, 2015; Wood, Tov, \& Costello, 2015). Documenting how people describe each other - how reputational knowledge is shared - thus has far-ranging theoretical implications.

We present here a network approach to the study of reputation. We extend the methodological and analytic tools of Kenny's (1994) interpersonal perception paradigm to study the questions raised by Craik's (2009) network interpretation of reputations. This provides a paradigm to study the flow of reputational information to people who have heard about, but never actually met, a target. In two studies, we apply this paradigm to study basic questions about consensus, accuracy, and positivity; how the personalities of people in the network and the context in which they are communicating affect the flow of information; and social consequences of reputations. 
NETWORK APPROACH TO REPUTATIONS

We begin by introducing the concept of reputation networks and provide an overview of the network approach to reputation, building on the dyadic approach.

\section{Reputation Networks and Interpersonal Perception}

In the most general terms, network analysis concerns the relations (called edges) between entities (called nodes), collectively referred to as a network (Kadushin, 2012). In Craik's (2009) network approach to reputations, the nodes represent people, specifically perceivers (which we label $P$ ) who know of some target (labeled $T$ ). The edges represent the flow of information about $\mathrm{T}$ between the nodes. Reputation networks are thus egocentric networks where $\mathrm{T}$ is the ego and Ps are the alters.

Figure 1 illustrates this idea with a simple, hypothetical reputational network. Some perceivers get information directly by observing or interacting with $\mathrm{T}$; we refer to these as firstdegree perceivers $(P 1 \mathrm{~s})$. Other perceivers get information secondhand, through hearsay from other perceivers; we refer to these as second-degree perceivers $(P 2 \mathrm{~s})$. One can imagine larger or more interconnected networks, but we limit our scope to these three positions (T, P1, and P2) of the reputational network in the current research. As depicted in Figure 1, we refer to impressions held by first-degree perceivers as the target's direct reputation, since information flows directly from $\mathrm{T}$ to $\mathrm{P} 1 \mathrm{~s}$. We refer to impressions held by second-degree perceivers as the target's hearsay reputation, since information flows indirectly from $\mathrm{T}$ to $\mathrm{P} 2$ through one or more $\mathrm{P} 1 \mathrm{~s}$, via the process of gossip or hearsay. 
NETWORK APPROACH TO REPUTATIONS

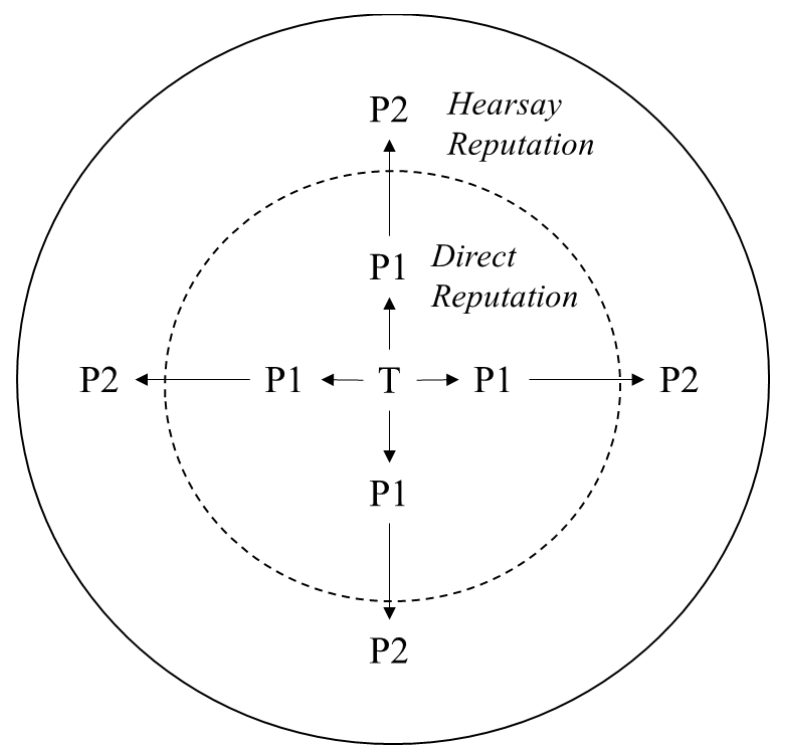

Figure 1. Graphical depiction of a generic reputational network. $\mathrm{T}=$ Target; $\mathrm{P} 1=$ first-degree Perceiver; P2 = second-degree perceiver.

The network approach to reputation builds on the dyadic approach in interpersonal perception, which includes two positions, a target and a first-degree perceiver (also sometimes referred to as self and other, or identity and reputation; see Funder, 1995; Hogan, 1996; Kenny, 1994, 2004; McAbee \& Connelly, 2016; Vazire, 2010). We extend this work by extending beyond T-P1 dyads and examining indirect information that is further shared from P1s to P2s. By comparing the impressions at each position - impressions held by targets (of their own self), P1s, and P2s - we can infer how information has flowed between those nodes. This approach has been used successfully in models like Funder's (1995) Realistic Accuracy Model (RAM), Vazire's (2010) Self-Other Knowledge Asymmetry (SOKA) model, and Kenny’s (1994) PERSON model, which use judgements by different perceivers to infer the underlying process of information flowing directly from target to perceiver. The present studies extend this approach to study information about targets held by second-degree perceivers, which we call hearsay reputations. 
NETWORK APPROACH TO REPUTATIONS

A change from the dyadic to network approach is the importance of triads in the network approach. Triads are often considered the basic building block of social networks more generally (Kadushin, 2012), and the T-P1-P2 triad is a fundamental unit of analysis for the network approach to reputations (analogously to the important role of target-perceiver dyads in the dyadic approach). Empirical paradigms within the dyadic approach tend to be designed for recruiting (e.g., through peer nominations) or creating (e.g., in round-robin designs) a set of targetperceiver dyads, with individuals sometimes serving multiple roles across overlapping conceptual dyads (as in round robin designs, where each subject is both a target and a perceiver in multiple overlapping dyads). Analogously, the empirical paradigm we introduce in the present studies consists of extending this from dyads to triads by creating naturalistic micronetworks of T-P1-P2 triads. In this paradigm, all three roles are occupied by real participants, not confederates or artificial stimuli such as vignettes or videos. We created the T-P1 link by having two people get to know each other in the first phase of the experimental session. In the second phase, we created the P1-P2 link by having each P1 tell a naïve person (P2) about the person they met. We use this basic approach to investigate consensus, accuracy, and positivity bias in information flowing across reputation networks, potential moderators of the flow of reputational information, and the social consequences of reputational information.

\section{Assessing the Flow of Reputational Information by Extending Interpersonal Perception}

The primary aim of these studies was to better understand the flow of reputational information through networks. We can compare the information "stored" as impressions held by people at different places in the network to understand how information traverses it. The extent to which information stored at each layer is similar to or different from other layers can be used to infer the strength, validity, and biases in information flowing through the networks. This is 
NETWORK APPROACH TO REPUTATIONS

analogous to how interpersonal perception researchers have studied the consistency of direct reputations (e.g., interjudge consensus; Connelly \& Ones, 2010; Kenny, 1994; Funder, Kolar, \& Blackman, 1995), their accuracy (Funder, 1995; Kenny, 1994; Vazire, 2010), and biases (e.g., self-enhancement; Kwan, John, Kenny, Bond, \& Robins, 2004) by comparing P1s’ impressions to each other and to Ts' self-reports.

To do this, we adopted and extended two approaches from the interpersonal perception approach. First, we used a multilevel profile analysis, the extended social accuracy model (SAM; Biesanz, 2010; Wessels et al., 2020). This approach compares the information stored at two nodes across multiple trait domains and derives two indices of agreement and one of directional bias. A normative component reflects agreement on what an average target is like; a distinctive component reflects agreement about how a specific target differs from an average one; and a positivity component reflects a perceiver's bias toward attributing evaluatively positive traits. In a second approach, we analyzed trait domains one at a time (i.e., extraversion, agreeableness, etc.). This allowed us to test hypotheses that focused on specific trait domains.

Consensus and positivity in hearsay reputations. "Consensus" refers to agreement between perceivers. Within a network approach it is possible to define different kinds of hearsay consensus, including P2-P2 consensus, P1-P2 consensus, and further variations (e.g., between Ps closer or further in network space). In the present study we only examined P1-P2 consensus, and so we will use the term "hearsay consensus" interchangeably with P1-P2 consensus.

The normative component of consensus reflects the extent to which perceivers agree on what an average target is like. Within the present approach, it is not possible to separate how much of this normative component reflects similarity in pre-existing, static background assumptions about people in general, as opposed to being produced dynamically through gossip. 
NETWORK APPROACH TO REPUTATIONS

By contrast, the distinctive component of consensus is agreement about the idiosyncratic characteristics of a particular target. When the only potential source of information that a P2 has about a target is a P1, then distinctive consensus will reflect information flow through the network from P1s to P2s. Our working hypothesis was that there would be evidence of both normative and distinctive components of consensus, reflecting information conveyed through gossip.

Within the extended SAM, a positivity component indicates that a perceiver has a bias toward making positive evaluations. We applied this to examine whether hearsay reputations are more or less positive than would be expected from P2s' agreement with P1s. Dyadic research has shown that knowing a target better is associated with less positively biased perceptions, suggesting that perceivers may fill in missing information with positivity (Wessels et al., 2020). If positivity is a function of missing information, then it is possible that $\mathrm{P} 2 \mathrm{~s}$ would be even more positively biased than P1s. It is also possible that P1s disproportionately share positive information about targets with P2s, stemming from the social cost of sharing negative gossip (Craik, 2009; Farley, 2011), which would also result in P2s having more positive impressions than P1s. Conversely, social interactions may engender more positive feelings than merely hearing about a person secondhand, resulting in P2s making more negative judgments, relative to P1s. In light of these differing perspectives, we treated the issue of the relative positivity of perceivers as an open question, for which analyses in the present data could be informative.

Accuracy at different layers of the network. Hearsay accuracy refers to the extent to which hearsay reputations are valid and reflects the amount of valid information transmitted from targets to second-degree perceivers (via first-degree perceivers). There is no gold-standard criterion for gauging accuracy; instead, a choice of criterion needs to be justified in the context 
NETWORK APPROACH TO REPUTATIONS

of a particular study (see Funder, 1995; Kenny, 1994; Robins \& John, 1997). We used targets' self-reports as the accuracy criterion in this study, a common approach in interpersonal perception research (e.g., Back et al., 2010, German Sample; Biesanz, 2010; Biesanz \& Human, 2010; Graham \& Gosling, 2012; Harari, Graham, \& Gosling, 2015; Human \& Biesanz, 2011, 2012). This reflected our assumption that it is defensible to treat self-perceptions of personality as containing enough valid information to be informative benchmarks for evaluating impressions made by strangers. We refer to T-P2 agreement as hearsay accuracy and T-P1 agreement as direct accuracy, since each measures the accuracy of hearsay and direct reputations respectively.

As with consensus, the normative components of both direct and hearsay accuracy can reflect either similarity in pre-existing, static background assumptions or dynamically formed impressions. Previous studies suggest that it is at least partially dynamic, since it increases as a function of knowing the target and decreases as a function of liking the target (Wessels et al., 2020; Zimmermann et al., 2018).

The distinctive component is closer to folk conceptions of accuracy and refers to the extent to which perceivers know how a specific target differs from an average one (Biesanz, 2010). In first-degree perceptions, distinctive accuracy has been found to increase as a function of acquaintanceship and perceivers' motivation to achieve accuracy (Biesanz \& Human, 2010; Wessels et al., 2020). If first-degree perceivers are forming accurate impressions and then conveying them to second-degree perceivers, we would expect hearsay accuracy to be better than chance. However, if that information is degraded as it traverses the network, we would expect hearsay accuracy to be weaker than direct accuracy.

Domain-wise consensus and accuracy. The profile approach of the extended SAM, described above, integrates over many different trait domains. We also examined consensus and 
NETWORK APPROACH TO REPUTATIONS

accuracy in different trait domains one at a time, analyzing associations between impressions of single traits (conceptually this corresponds most closely to the distinctive component in the SAM, but for a single trait). Doing so provides a more granular understanding of consensus and accuracy for specific traits. The Realistic Accuracy Model (RAM; Funder, 1995) proposes that information flows differently from targets to perceivers for different traits. This is born out metaanalytically: both consensus and accuracy vary considerably depending upon which personality trait is being perceived, highlighting the importance of the domain-wise approach (Connelly \& Ones, 2010). Another advantage of a domain-wise approach is that it has been more widely used in interpersonal perception research, making it possible to interpret the present findings in relation to a larger body of existing research.

Previous research on first-degree perceivers using this approach has found that consensus is generally higher than accuracy (Connelly \& Ones, 2010). One reason P1-P1 agreement exceeds self-P1 agreement is that P1s share many of the motivational and informational factors that might bias perceptions with each other, but not with the self(Vazire, 2010). P1s and P2s may have different motivations, and certainly have access to different kinds of information (direct vs. indirect). These differences could dampen hearsay consensus.

However, there are reasons to expect that agreement between connected P1s and P2s may be stronger, not weaker, than P1-P1 agreement. In Kenny's (2004) PERSON model, one component of an interpersonal judgment, labeled opinion, is a part of a judgment that is stable but idiosyncratic to that perceiver: it can be thought of as that perceiver's unique "take" on the target. Work with the PERSON model suggests that the opinion component is a sizeable part of any single perceiver's impression, and perceivers are unaware of what part of their impression belongs to each component. Two first-degree perceivers will (by definition) have different 
NETWORK APPROACH TO REPUTATIONS

opinion components, which will lead to lower consensus between them. However, when one perceiver communicates their impression to another perceiver through gossip, they will include the opinion component as a part of that, potentially leading to greater agreement. We did not have a direct measure of P1-P1 agreement in this study, but we used meta-analytic estimates (Connelly \& Ones, 2010) as interpretive benchmarks to compare with P2-P1 agreement.

\section{What Moderates the Flow of Reputational Information?}

A variety of factors may influence the strength of hearsay consensus and accuracy, including acquaintanceship, relationship type, and more. A systematic examination of all these potential factors was well beyond the scope of this investigation. However, as a beginning step, we studied two such factors: individual differences among different members of the network, and in study 2, the context of the hearsay exchange between P1 and P2.

Individual differences. Based on previous research examining good judges of personality and good (easily judged) targets (Funder 1995; Human \& Biesanz, 2013), we investigated whether individual differences among targets, P1s, and P2s could impact hearsay consensus and accuracy. We specifically examine extraversion (among targets, P1s, and P2s) and empathy (among P2s only) as moderators of hearsay consensus and accuracy. Targets' extraversion has been found to promote accurate interpersonal perception, presumably via extraverts' heightened talkativeness and expressivity, which makes a greater number of cues available to perceivers (Funder, 1995; Human \& Biesanz, 2013). More speculatively, P1s’ extraversion may influence how much they say about the target, or P2s' extraversion may lead them to elicit more information in a conversation. We also examined P2s' empathy as a moderator. This was based on the intuition that more empathic P2s may be better at drawing inferences from what P1s say about the target. Given the broad, cross-domain nature of these 
NETWORK APPROACH TO REPUTATIONS

theories (i.e., that extraverted targets express more cues generally), we investigated individual differences as moderators of the broad SAM components.

Context and hearsay reputations. We hypothesized that one important part of the context of the hearsay exchange - the stated goal of the conversation where first-degree perceivers told second-degree perceivers about the target - could affect hearsay consensus and accuracy. Personality domains are not all equally relevant to all goals or contexts (Cottrell et al., 2007; see also Buss, 2011; Srivastava, 2010; Wood, 2015). In conversations, Grice’s (1975) maxim of relation reflects a norm that individuals should provide information that is relevant to the topic at hand. Taken together, this suggests that individuals may provide more information about personality domains that are relevant to the context in which the hearsay discussion takes place.

The specific contexts we examined in Study 2 were goals provided to the participants to evaluate the target's suitability for status- or affiliation-related roles (leadership and friendship respectively). Status and affiliation, or getting ahead and getting along, are two critical and ubiquitous demands of social living (Hogan, 1996). Within the Big Five, extraversion is associated with status or leadership and predicts both status attainment and leadership ability (Anderson, John, Keltner, \& Kring, 2001; Judge, Bono, Ilies, \& Gerhardt, 2002; Lawless Desjardins, Srivastava, Küfner, Back, 2015). Affiliation, on the other hand, is theorized to be most strongly connected to agreeableness, both in terms of intimate (affiliative) relationships and getting along or cooperating with others more generally (Buss, 1996, 2011; Denissen \& Penke, 2008; DeYoung, 2015). Supporting this notion, people tend to like others higher in agreeableness (Wortman \& Wood, 2011), and report valuing agreeableness in friendships and other intimate (affiliative) relationships (Cottrell et al., 2007; Wood, 2015). Due to the domain-specificity of 
NETWORK APPROACH TO REPUTATIONS

these hypotheses, we examine context as a moderator of domain-wise correlations for extraversion and agreeableness.

\section{Social Consequences of Hearsay Reputations}

One motivating assumption of studying hearsay reputations is that they have consequences for how the people involved treat one another. Indeed, our context manipulations were built on the assumption that conversations would center on role-relevant information because people use this information in their judgments of the targets' fitness for those roles. We pursued this directly by testing two hypotheses concerning the social consequences of information flowing through reputation networks. Specifically, we assessed the extent to which the information flowing from P1 to P2 about personality traits affected relevant social decisions P2 might make about the target. We hypothesized that information about the targets' extraversion flowing from P1 to P2 would affect the extent to which P2 thinks the target would make a good leader. We also hypothesized that information about the targets' agreeableness flowing from P1 to P2 would affect the extent to which P2 thinks the target would make a good friend.

\section{The Present Studies}

The present studies were designed to provide a first look at hearsay reputations within this paradigm. We present the paradigm and an associated data-analytic framework that extends methodological and analytic approaches designed for interpersonal perception (e.g., Biesanz, 2010; Kenny, 1994; Olsen \& Kenny, 2006; Wessels et al., 2020). In the first phase of the procedure, two previously unacquainted people get to know one another. In the second phase, one or both of them tell new participants about the person they just met. These two phases create an experimental instantiation of a basic reputational network (as depicted in Figure 1). We 
NETWORK APPROACH TO REPUTATIONS

originally conducted two separate studies, with the second being a replication and extension with a preregistered plan to combine datasets where appropriate. In the combined analyses, we examine consensus, accuracy, and bias in hearsay reputations using both the componential SAM and domain-wise approach, and we examine individual-difference moderators of consensus and accuracy. In Study 2, we examine the experimentally manipulated context moderators, and we examine how social decisions made about the target were influenced by hearsay reputations of Big Six personality characteristics.

\section{Method}

\section{Preregistration}

The measures and materials, procedure, sample size, exclusion criteria, and analysis plan for both studies were preregistered on the Open Science Framework (OSF; preregistration for Study 1: https://osf.io/hqtfb; preregistration for Study 2: https://osf.io/kfcmt; full project site: https://osf.io/65wfu). The full procedure was carried out as described in the preregistration, except where noted in this manuscript.

Analyses were preregistered as primary or secondary, reflecting their centrality to the investigation. We preregistered a plan to report all primary analyses but only selected secondary analyses. Primary analyses in Study 1 included hearsay consensus and accuracy for the Big Six. Primary analyses in Study 2 included replicating the Study 1 analyses in a combined dataset (the Integrated analyses) and tests of the hypotheses outlined above regarding the context manipulation and social consequences.

Deviations from preregistrations. We decided to deviate from our preregistered analysis plan in two significant ways based on feedback from peer review. First, we dropped the Study 1 analyses from the manuscript entirely because the results did not significantly differ 
NETWORK APPROACH TO REPUTATIONS

from those from the replication analyses in Study 2, making them less precise but otherwise redundant with integrated analyses on the combined Study 1 and Study 2 data (which were part of Study 2's preregistration). Second, we incorporated the extended SAM analyses (Biesanz, 2010; Wessels et al., 2020). The extended SAM affords greater power and parsimonious tests of consensus, accuracy, and positivity bias that are integrated across items and personality domains. When the extended SAM addresses the same conceptual questions as the preregistered analyses, we only report the former. A preprint of the original manuscript with all of the primary preregistered analyses is available on PsyArxiv (https://psyarxiv.com/8wg25).

\section{Participants}

We recruited two separate samples, one for each study, which we ultimately combined for most analyses. Both studies were approved by the University of Oregon's institutional review board (Protocol \# 12032015.004). The preregistered sampling plan for Study 1 was to collect data until we had 100 targets (between 50 and 100 groups of 3 and 4 participants). The preregistered sampling plan for Study 2 was to collect data until we had at least 120 targets (between 60 and 120 groups of 3 and 4 participants). For both, we planned to continue collecting data if it was minimally costly (e.g., continuing data collection until the end of the academic term). In both cases, we based our minimum sample sizes based on available resources and achieving roughly $80 \%$ power to detect a correlation of $.3^{1}$.

Both studies had three exclusion criteria. We conducted a blinded screen for invalid responding, which was preregistered for both studies. We excluded groups where participants knew each other, which was registered as an addendum to the study 1 preregistration shortly after data collection began (https://osf.io/eckj7/) and preregistered for study 2. And we excluded

\footnotetext{
${ }^{1}$ Power is less straightforward in a design like the present, so we based our power analysis on a basic, bi-variate correlation as a rough approximation.
} 
NETWORK APPROACH TO REPUTATIONS

individuals who reported that they did not understand the instructions; this was inspired by unanticipated incidents in Study 1 and included in the Study 2 preregistration.

Study 1. Participants were recruited for the study from the University of Oregon Human Subjects Pool and were unaware of the topic of the study upon signing up. Participants signed up independently and participated in groups of 3 or 4 . The initial sample consisted of 288 participants. We eliminated 7 groups where participants knew each other, and an additional group was unable to finish the study due to a mandatory evacuation in the building. Three participants were excluded after they reported to the researcher(s) that they did not follow the questionnaire instructions. This resulted in a final sample of 260 participants in 72 groups. The final sample included 115 participants in the target role, consistent with the preregistered plan.

Participants were aged 18 to 30 , with an average age of $19.6(S D=1.8)$. The majority of participants identified as women ( $58 \%$ of those that reported; $15 \%$ did not report gender). The majority of participants identified as White/Caucasian (60\%), followed by Asian (18\%), Hispanic/Latino (9\%), other (4\%), Black/African American (4\%), Native American (<1\%), and $15 \%$ did not report race/ethnicity; participants were able to select more than one option.

Study 2. We again recruited participants from the University of Oregon Human Subjects Pool. The initial sample consisted of 381 participants in 108 groups. Three groups were excluded for knowing each other, and one additional participant was excluded for not following instructions. This resulted in a final sample of 369 participants in 105 groups (50 groups of 3; 55 groups of 4). The final sample included 160 participants in the target role, consistent with the preregistered plan.

Participants were aged 18 to 32 , with an average age of $19.4\left(S D_{\text {Age }}=1.8\right)$. The majority of participants identified as women (59\% of those that reported). The majority of participants 
NETWORK APPROACH TO REPUTATIONS

identified as White/Caucasian (62\%), followed by Asian (14\%), Hispanic/Latino (11\%),

Black/African American (5\%), other (3\%), Native American (2\%); participants were able to select more than one option. $14 \%$ of participants did not complete the demographic questions.

\section{Procedure}

The full procedure, script, and instructions can be found at the preregistration link (Study 1: https://osf.io/hqtfb; Study 2: https://osf.io/kfcmt). Participants were scheduled in groups of 3 or 4. They were instructed to arrive and wait in separate areas to ensure that they had little to no contact prior to the start of the experiment. Before the start of the experiment, participants were randomly assigned to the different procedural 'roles', which corresponded to one or more of the network positions outlined above (T, P1, P2). Figures $2 \mathrm{a}$ and $2 \mathrm{~b}$ show how the triads (Figure 2a) and tetrads (Figures 2b) of participants map onto the theoretical roles of interest. As shown in Figure $2 \mathrm{a}$, each participant in a three-person group served one role, completing a single reputational triad ${ }^{2}$, with one participant serving the target role, one serving the P1 role, and one serving the P2 role. As shown in Figure 2b, four-person groups were more complex: two participants were simultaneously targets and P1s and two participants were P2s (see Figure 2b). Four-person groups thus contained two overlapping reputational triads. Experimenters retrieved participants, one at a time, and directed them to an empty lab room where they were instructed to read the informed consent document and await the start of the experiment. From this point, the experiment proceeded in three phases: 1) dyadic interaction, 2) hearsay, and 3) ratings.

\footnotetext{
${ }^{2}$ The target and P1 did rate each other, making them simultaneously a target and perceiver of each other. However, each played a single role with respect to the T-P1-P2 reputational triad.
} 
NETWORK APPROACH TO REPUTATIONS

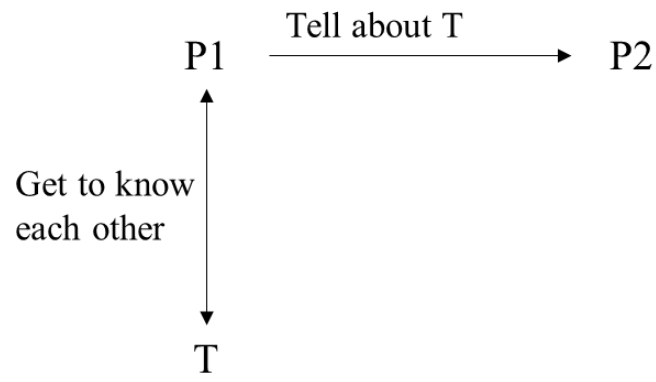

(a) Three-person group

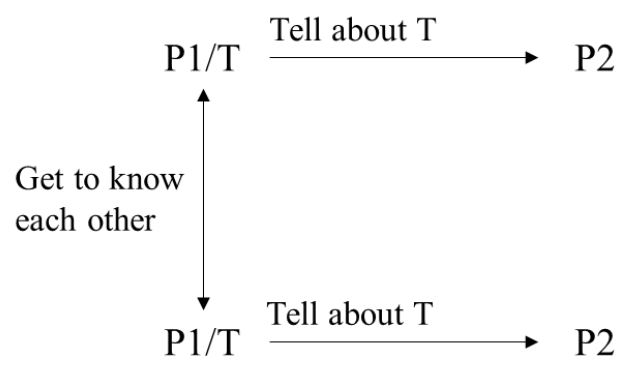

(b) Four-person group

Figures 2a-b. Graphical depiction of study design. Participant roles appear in parentheses below the name.

Phase 1: Dyadic interaction. Two participants were brought into a lab room and sat at opposite sides of a table that had a stack of cards at the center of it. The experimenter asked if the participants knew one another. Then, the experimenter instructed them that they were to play a get-to-know-you game, taking turns asking questions printed on the cards to which both were expected to respond. Questions were written to reveal broadly relevant personality information. We included 12 questions that were each targeting a Big Six domain (2 questions per domain). For example, "What do you do to manage schoolwork and deadlines?" was intended to target conscientiousness. We included 6 additional questions eliciting small talk (one from the control condition of Aron et al., 1997). Participants were instructed to go through the questions in order and to get through as many of the questions as they could in the 15 minutes they were allotted. These conversations were recorded, and participants were aware of these recordings.

Meanwhile, the remaining participant(s) were seated in front of their own computer (alone) and completed a questionnaire consisting of the Interpersonal Reactivity Index (Davis, 1983) and filler questions for 15 minutes.

Phase 2: Hearsay. In the second phase, one or both of the participants from the phase-1 interaction had a conversation with a new participant about the person they had just met. In 
NETWORK APPROACH TO REPUTATIONS

sessions run with 3-person groups, one person from the phase-1 interaction was assigned to the role of P1 and met with a new participant serving as a P2. In 4-person groups, each participant from the phase- 1 interaction now served as a $\mathrm{P} 1$ describing their previous interaction partner to a new P2. P1s were instructed to tell the new person (P2s) about the person whom they had just met. They were instructed to, “...give the most informative, objective, and accurate account of what the person you [they] met was like," and P2s were similarly instructed to, "try to get the most accurate impression you [they] can from what [P1] tells you [them]." These conversations were also recorded; participants were aware of the recordings.

Context Manipulation in Study 2. Phase 2 of the experiment was the only part of the procedure that differed between studies. In Study 2, participants in the status and affiliation conditions were read the following instructions at the beginning of Phase 2:

We want you to talk about what that person is like in general. Later on, we are going to ask [P2] about [his or her] impressions of that person. One thing we are going to ask is whether [P2] thinks the person you're describing would make a (good leader / good friend who they would want to get close to). So we want you to help [P2] form an overall impression, which includes forming an impression of that person's (leadership potential / potential as a friend).

In the control condition, the italicized sections above were removed. Outside of this and the additional measures administered after the interactions, the procedure was identical to Study 1.

Phase 3: Ratings. After approximately 10 minutes had passed, participants were separated and instructed to complete a series of questionnaires on a computer. After finishing, participants were thanked and debriefed. 
NETWORK APPROACH TO REPUTATIONS

Measures. Questionnaires included self-, P1-, and P2-reported personality questionnaires as relevant to each participant's roles (described below), followed by a brief set of demographic questionnaires. Participants in the P2 role additionally completed open-ended descriptions of the person they heard about; these were not analyzed for this manuscript.

Big Six. We administered self-, P1-, and P2-reports of the Big Six personality domains. We used the full (60-item) Big Five Inventory 2 (BFI-2; Soto \& John, 2017) to measure the Big Five domains, which are agreeableness, conscientiousness, neuroticism, extraversion, and openness. We used eight items from the 48 Questionnaire Big Six (48QB6; Thalmayer, Saucier, \& Eigenhuis, 2011) to measure honesty-propriety. All ratings were completed on a scale ranging from 1 (Disagree Strongly) to 5 (Agree Strongly) with a neutral point of 3 (Neutral; no opinion). The only difference between self-, P1-, and P2-reports were the instructions, which were appropriately re-worded to reflect the target they were rating.

Coefficient alphas for each of the domain scales of the Big Six are reported in Supplemental Table S1 and summarized here. Among full scales, honesty-propriety consistently had the lowest alpha value (.61, .66, and .75 for self, P1-, and P2-reports respectively in Study 1; $.62, .76$, and .79 for self, P1-, and P2-reports respectively in Study 2); this may be because it had the fewest items ( 8 for honesty-propriety vs. 12 for each of the Big Five). All other alpha coefficients were above .70 .

Big Six desirability profile. For the positivity analyses, we collected desirability ratings for all 68 items from 13 expert judges; judges consisted of undergraduate research assistants and graduate students familiar with the Big Six and with the concept of social desirability. For each item, judges rated the extent to which 'a description of a person with this item implies a positive or negative evaluation?' on a scale from 1 (Very Negative) to 5 (Very Positive) with a neutral 
NETWORK APPROACH TO REPUTATIONS

point of 3 (Neutral). Desirability ratings were highly consistent across judges, with an ICC for the average (i.e., ICC2k) of .97 and an ICC for a single random rater (i.e., ICC2) of .74. We took the average across all 13 judges and used this as the item positivity profile in extended SAM analyses. Average desirability ratings are shown alongside average self, P1, and P2 endorsement in Supplemental Table S2.

Empathy. Participants in the P2 role completed the Interpersonal Reactivity Index (IRI; Davis, 1983), which measures four aspects of empathy (perspective taking, personal distress, fantasy, and empathic concern) with 28 short statements (e.g., "I believe that there are two sides to every question and try to look at them both" from Perspective Taking) that are rated on a 0 (Does not describe me well) to 4 (Describes me very well) scale. All four subscales demonstrated adequate internal consistency ( $\alpha$ 's from .70 to .82).

Social decisions. In Study 2 we added two additional single items for P1- and P2-reports. Participants rated the extent to which they thought the target would be a good leader and good friend. These items were on the same 1 to 5 scale as other items administered.

\section{Analytic Approach}

In this design, individuals are nested in groups, creating 3- or 4-person reputational micro-networks designed to emulate a reputational network. Figure 1 depicts three kinds of roles in a theoretical reputational network; Figures $2 \mathrm{a}$ and $2 \mathrm{~b}$ show how these roles map onto the 3and 4-person micronetworks in the study design. Our data analytic approach draws on concepts and principles from dyadic data analysis (e.g., Actor Partner Interdependence Models; APIM; Olsen \& Kenny, 2006; SAM; Biesanz, 2010) and applies them to 3- and 4-person micronetworks. 
NETWORK APPROACH TO REPUTATIONS

Profile analyses with SAM. We modeled dependencies in the SAM by treating participants as nested within triads and groups. In 3-level mixed effects models, we treated each response on item $i$ as nested in T-P1-P2 triad $t$, which in turn was nested in group $g^{3}$. All SAMs contained fixed effects for a normative response profile (Normative ${ }_{i}$ ), a distinctive profile for that target (Distinctive $\mathrm{i}_{\mathrm{i}}$ ), and the profile of item positivity (Positivity $)$. All ratings were grand mean centered before any further calculations. Normative profiles were estimated by calculating the average per item across ratings for either self-reports (in the case of hearsay accuracy) or P1 reports (in the case of hearsay consensus) on each item. Distinctive profiles were estimated by subtracting the normative profile from each individual self-report (hearsay accuracy) or P1report (hearsay consensus). We used the Satterthwaite approximation to calculate degrees of freedom for all tests of fixed effects. All intercepts and slopes were always allowed to vary across triads and groups and covary within level (i.e., random effects for triads could co-vary; random effects for groups could co-vary).

Domain-wise correlations. Domain-wise correlations were estimated using an extension of dyadic path analysis (e.g., Actor Partner Interdependence Models; APIM; Olsen \& Kenny, 2006). Two concepts were central to our approach to modeling domain-wise correlations. First is the concept of dependencies. We modeled dependencies in the 3- or 4-person micro-networks by treating individuals as nested in triads and tetrads. Second is the concept of pooling interchangeable estimates. In APIM, this means pooling means, variances, and paths (regression paths representing actor and partner effects) across dyad members when they are exchangeable. Our design is more complex, but the exchangeability principle applies in a similar manner. We pooled estimates of the means and covariances of ratings whenever the people making those

\footnotetext{
${ }^{3}$ Note that $\mathrm{t}$ and $\mathrm{g}$ are identical in 3-person groups, but there are two observations of $t$ per $g$ in 4-person groups.
} 
NETWORK APPROACH TO REPUTATIONS

ratings occupied the same theoretical roles $(\mathrm{T}, \mathrm{P} 1$, or $\mathrm{P} 2)$ and were procedurally exchangeable (i.e., did the same thing in the experiment). Supplemental Table S3 contains an example variance-covariance matrix with equality constraints noted in its cells.

Integrating across studies. As preregistered, we conducted analyses on a dataset that combined the two studies, thus providing a single integrated estimate for each parameter of interest (e.g., distinctive hearsay consensus, hearsay consensus for extraversion, etc.). These integrated estimates are the only results shown in the present manuscript. We assessed the justifiability of integrating across studies for both the SAM and domain-wise correlation analyses by comparing a model that estimated a single parameter for both studies to one that allowed model parameters to differ across studies. The results of these analyses, reported in the supplemental materials, supported combining results across studies.

Inference criteria. As noted in our preregistration for both studies, our focus was on effect size and precision. We present $95 \%$ confidence intervals around each parameter we estimate, and to aid interpretation, we compare the effect sizes we observe in our study to benchmarks from previous literature whenever possible (i.e., meta-analytic estimates taken from Connelly \& Ones, 2010 for domain-wise correlational analyses). We also present exact $p$ values and use significance tests to help rule out that the effects arise from chance guessing alone (e.g., zero consensus or accuracy). We used two-tailed significance tests with alpha $=.05$ as the cutoff, as preregistered.

R packages. All mixed effects models were run in lme4 (Version 1.1-21; Bates, Maechler, Bolker, \& Walker, 2015) and used lmerTest (Version 3.1-1; Kuznetsova, Brockhoff, \& Christensen, 2017) for significance tests of fixed effects. Path models were run using the lavaan package (Version 0.6-3; Rosseel, 2012) and our own ReputationModelR package (see: 
NETWORK APPROACH TO REPUTATIONS

https://github.com/Coryc3133/ReputationModelR) in R (version 3.6.2; R Core Team, 2019). The data and a script that reproduces the analyses can be found at our OSF project page

(https://osf.io/65wfu).

\section{Results}

\section{Extended SAM Analyses}

To what extent did second-degree perceivers (P2s) agree with first-degree perceivers (P1s), reflecting consensus? How much did P2s agree with the self-reports of targets (Ts), reflecting accuracy? Did they show positivity bias? We conducted extended SAM analyses to answer these questions.

Hearsay consensus with the SAM. For hearsay consensus, we estimated each P2's rating of the target on item $i$ as a function of the average $\mathrm{P} 1$ rating of targets on that item (Normative $\mathrm{P} 1_{\mathrm{i}}$ ), how a $\mathrm{P} 1$ deviated from the average in their rating of a target (DistinctiveP $1_{\text {it }}$ ), and the item's positivity (Positivityi). This allowed us to simultaneously estimate normative consensus, distinctive consensus, and positivity bias. We used the following equation:

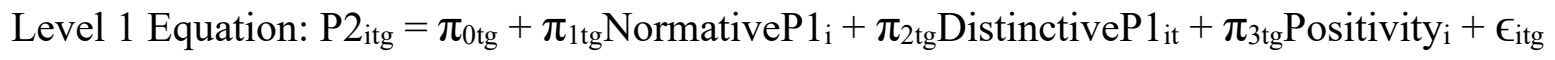
Level 2 Equation: $\pi_{0 \operatorname{tg}}=\beta_{00 \mathrm{~g}}+\mathrm{r}_{0 \mathrm{tg}}$

$$
\begin{aligned}
& \pi_{1 \mathrm{tg}}=\beta_{10 \mathrm{~g}}+\mathbf{r}_{1 \mathrm{tg}} \\
& \pi_{2 \mathrm{tg}}=\beta_{20 \mathrm{~g}}+r_{2 \mathrm{tg}} \\
& \pi_{3 \mathrm{tg}}=\beta_{30 \mathrm{~g}}+r_{2 \mathrm{tg}}
\end{aligned}
$$

Level 3 Equation: $\beta_{00 \mathrm{~g}}=\gamma_{000}+\mathrm{u}_{00 \mathrm{~g}}$

$$
\begin{aligned}
& \beta_{10 \mathrm{~g}}=\gamma_{100}+\mathrm{u}_{10 \mathrm{~g}} \\
& \beta_{20 \mathrm{~g}}=\gamma_{200}+\mathrm{u}_{20 \mathrm{~g}} \\
& \beta_{30 \mathrm{~g}}=\gamma_{300}+\mathrm{u}_{30 \mathrm{~g}}
\end{aligned}
$$

Fixed effects are shown in Table 1 (see Supplemental Table S4 for random effects). The results showed a large degree of normative consensus, a moderate degree of distinctive 
NETWORK APPROACH TO REPUTATIONS

consensus, and a very small degree of positivity bias that is indistinguishable from zero. This suggests that P2s agreed with P1s in how the targets were similar to the average target and also in how individual targets distinctly differed from the average target. After accounting for these components, there was little evidence that P2s saw the target more or less positively than P1s did. Thus, general and target-specific information appeared to flow robustly from P1s to P2s, and this information did not appear positively or negatively biased.

Table 1. Fixed effects from Hearsay Consensus SAM

\begin{tabular}{llcccccccc}
\hline term & component & Est. & CI LL & CI UL & SE & $t$ & $d f$ & $p$ & Std. Est \\
\hline$\gamma_{000}$ & Intercept & 0.00 & -0.02 & 0.02 & 0.01 & 0.00 & 164.82 & .998 & .00 \\
$\gamma_{100}$ & Normative Consensus & 0.73 & 0.66 & 0.80 & 0.04 & 20.00 & 183.37 & $<.001$ & .46 \\
$\gamma_{200}$ & Distinctive Consensus & 0.20 & 0.18 & 0.23 & 0.01 & 16.53 & 186.83 & $<.001$ & .18 \\
$\gamma_{300}$ & Positivity Bias & 0.05 & 0.00 & 0.09 & 0.02 & 1.92 & 233.82 & .056 & .05 \\
\hline
\end{tabular}

Note. Term refers to the term in the corresponding equation. Component refers to the SAM component. Est. refers to the unstandardized estimate. CI LL and CI UL refer to the lower and upper limits of the $95 \%$ CI respectively. SE refers to standard error. $d f$ were calculated using the Satterthwaite approximation. Std. Est. refers to the standardized estimates.

Hearsay accuracy with SAM. We simultaneously estimated hearsay and direct accuracy in the same model. To do this, we ran a SAM predicting perceptions from both first- and seconddegree perceivers, with a dummy-coded indicator variable to indicate which was which. At level 1 , we treated each perceiver's rating of the target on item $i$ as a function of the average selfrating for that item (NormativeSelf $\mathrm{f}_{\mathrm{i}}$, how a particular target deviated from the average in their self-rating for that item (DistinctiveSelf $f_{i t}$ ), and the item's desirability (Positivity $)$. At level 2, we modeled the effect of perceiver position or distance (P1 vs. P2) on the intercept $\left(\pi_{0 \mathrm{tg}}\right)$, slope for normative accuracy $\left(\pi_{1 \mathrm{tg}}\right)$, slope for distinctive accuracy $\left(\pi_{2 \mathrm{tg}}\right)$, and slope for positivity bias $\left(\pi_{3 \mathrm{tg}}\right)$. We used the following equations: 
NETWORK APPROACH TO REPUTATIONS

Level 1 Equation: $\mathrm{P}_{\mathrm{itg}}=\pi_{0 \mathrm{tg}}+\pi_{1 \mathrm{tg}}$ NormativeSelfi $+\pi_{2 \mathrm{tg}}$ DistinctiveSelf $_{\mathrm{it}}+\pi_{3 \mathrm{tg}}$ Positivity $_{\mathrm{i}}+\epsilon_{\mathrm{itg}}$

Level 2 Equation: $\pi_{0 \mathrm{tg}}=\beta_{00 \mathrm{~g}}+\beta_{01 \mathrm{~g} D}$ istance $_{\mathrm{tg}}+\mathrm{r}_{0 \mathrm{tg}}$

$$
\begin{aligned}
& \pi_{1 \mathrm{tg}}=\beta_{10 \mathrm{~g}}+\beta_{11 \mathrm{~g}} \text { Distance }_{\mathrm{tg}}+\mathrm{r}_{1 \mathrm{tg}} \\
& \pi_{2 \mathrm{tg}}=\beta_{20 \mathrm{~g}}+\beta_{21 \mathrm{~g}} \text { Distance }_{\mathrm{tg}}+\mathrm{r}_{2 \mathrm{tg}} \\
& \pi_{3 \mathrm{tg}}=\beta_{30 \mathrm{~g}}+\beta_{31 \mathrm{~g}} \text { Distance }_{\mathrm{tg}}+\mathrm{r}_{2 \mathrm{tg}}
\end{aligned}
$$

Level 3 Equation: $\beta_{00 \mathrm{~g}}=\gamma_{000}+\mathrm{u}_{00 \mathrm{~g}}$

$$
\begin{aligned}
& \beta_{01 \mathrm{~g}}=\gamma_{010}+\mathrm{u}_{01 \mathrm{~g}} \\
& \beta_{10 \mathrm{~g}}=\gamma_{100}+\mathrm{u}_{10 \mathrm{~g}} \\
& \beta_{11 \mathrm{~g}}=\gamma_{110}+\mathrm{u}_{11 \mathrm{~g}} \\
& \beta_{20 \mathrm{~g}}=\gamma_{200}+\mathrm{u}_{20 \mathrm{~g}} \\
& \beta_{21 \mathrm{~g}}=\gamma_{210}+\mathrm{u}_{21 \mathrm{~g}} \\
& \beta_{30 \mathrm{~g}}=\gamma_{300}+\mathrm{u}_{30 \mathrm{~g}} \\
& \beta_{31 \mathrm{~g}}=\gamma_{310}+\mathrm{u}_{31 \mathrm{~g}}
\end{aligned}
$$

The fixed effects from this model are shown in Table 2 (see Supplemental Table S5 for random effects). The main effects showed that perceivers demonstrated substantial normative accuracy, moderate distinctive accuracy, and substantial positivity bias. The negative coefficients for the interactions showed that each of these effects decreased as a function of distance from the target: normative accuracy decreased moderately, distinctive accuracy decreased slightly, and positivity bias decreased even less (with the lower limit of its CI bordering on no change). Together, these results suggest that $\mathrm{P} 2 \mathrm{~s}$, relative to $\mathrm{P} 1 \mathrm{~s}$, perceive targets as less like the average person, are less accurate about how the targets deviate from the average person, and are slightly less positively biased.

Table 2. Fixed effects from Hearsay and Direct Accuracy SAM

\begin{tabular}{llcccccccc}
\hline term & \multicolumn{1}{c}{ component } & Est. & CI LL & CI UL & SE & $\boldsymbol{t}$ & $\boldsymbol{d f}$ & $\boldsymbol{p}$ & Std. Est \\
\hline$\gamma_{000}$ & Intercept & 0.00 & -0.01 & 0.02 & 0.01 & 0.19 & 253.48 & 0.849 & 0.00 \\
$\gamma_{100}$ & Normative Accuracy & 0.45 & 0.41 & 0.49 & 0.02 & 22.48 & 210.50 & $<.001$ & 0.30 \\
$\gamma_{200}$ & Distinctive Accuracy & 0.11 & 0.10 & 0.13 & 0.01 & 13.15 & 214.07 & $<.001$ & 0.11
\end{tabular}


NETWORK APPROACH TO REPUTATIONS

\begin{tabular}{llcccccccc}
\hline term & \multicolumn{1}{c}{ component } & Est. & CI LL & CI UL & SE & $\boldsymbol{t}$ & $\boldsymbol{d f}$ & $\boldsymbol{p}$ & Std. Est \\
\hline$\gamma_{300}$ & Positivity Bias & 0.30 & 0.26 & 0.33 & 0.02 & 17.16 & 206.77 & $<.001$ & 0.31 \\
$\gamma_{400}$ & Distance & 0.00 & -0.02 & 0.02 & 0.01 & 0.00 & 188.11 & 0.998 & 0.00 \\
$\gamma_{110}$ & $\begin{array}{l}\text { Distance X } \\
\text { Normative Accuracy }\end{array}$ & -0.11 & -0.16 & -0.06 & 0.02 & -4.50 & 235.27 & $<.001$ & -0.05 \\
$\gamma_{210}$ & $\begin{array}{l}\text { Distance X } \\
\text { Distinctive Accuracy }\end{array}$ & -0.04 & -0.06 & -0.02 & 0.01 & -4.26 & 230.07 & $<.001$ & -0.03 \\
$\gamma_{310}$ & $\begin{array}{l}\text { Distance X } \\
\text { Positivity Bias }\end{array}$ & -0.05 & -0.09 & 0.00 & 0.02 & -1.99 & 270.64 & 0.048 & -0.03 \\
\hline
\end{tabular}

Note. Term refers to the term in the corresponding equation. Component refers to the SAM component. Est. refers to the unstandardized estimate. CI LL and CI UL refer to the lower and upper limits of the $95 \%$ CI respectively. SE refers to standard error. $d f$ were calculated using the Satterthwaite approximation. Std. Est. refers to the standardized estimates. Distance was dummy coded such that P1s were the reference group, so each interaction represents the difference in P2s' (relative to P1s') slopes.

The reductions in P2s' accuracy and positivity bias, while significant, were quite small.

We next examined whether the simple slopes for P2 remained significant by running a SAM predicting just P2 responses. All three fixed effects were significant, suggesting that P2s achieved normative accuracy (unstandardized slope $=0.34$ [95\% CI: 0.30, 0.38], standardized $=$ $.24, \mathrm{p}<.001$ ) and distinctive accuracy (unstandardized slope $=0.07$ [95\% CI: 0.05, 0.09], standardized $=.07, \mathrm{p}<.001$ ), and demonstrated a moderate positivity bias (unstandardized slope $=.26$ [95\% CI: $0.21,0.30]$, standardized $=.28, \mathrm{p}<.001)$; random effects can be seen in Supplemental Table S6.

\section{Hearsay Consensus and Accuracy using Domain-Wise Correlations}

We next present consensus and accuracy analyses using domain-wise correlations, which complement the profile-based SAM approach by providing deeper insight into differences in consensus and accuracy for each Big Six domain. 


\section{NETWORK APPROACH TO REPUTATIONS}

Domain-wise hearsay consensus. Domain-wise hearsay consensus was assessed by calculating the correlations between P1s' and P2s' ratings of the targets, separately for each of the Big Six. These correlations speak to the strength (but not necessarily accuracy) of information about targets flowing from P1s to P2s for each Big Six domain. Figure 3 presents hearsay consensus estimated in each study separately and combined (i.e., integrated), which are plotted alongside a meta-analytic benchmark (i.e., interjudge consensus for previously unacquainted participants; Connelly \& Ones, 2010). As seen in Figure 3, hearsay consensus was highest for extraversion and quite strong, $r=.62,95 \% \mathrm{CI}[.55, .69] p<.001$. It was moderately high for agreeableness $(r=.28,95 \%$ CI $[.17, .39], p<.001)$, conscientiousness $(r=.39,95 \% \mathrm{CI}$ $[.29, .49] p<.001)$, honesty-propriety $(r=.29,95 \% \mathrm{CI}[.18, .40] p<.001)$, neuroticism $(r=.34$, $95 \%$ CI $[.24, .44] p<.001)$, and openness $(r=.25,95 \% \mathrm{CI}[.15, .36] p<.001)$.

Hearsay consensus was similar in magnitude to meta-analytic estimates of interjudge consensus in most domains. The exception was extraversion, which was considerably higher than other domains and higher than its meta-analytic benchmark. Indeed, the lower bound of its $95 \%$ CI was greater than the meta-analytic benchmark and greater than the upper limits of the $95 \%$ CIs for other domains. Hearsay thus lead to consensus generally, but consensus for extraversion at levels that exceeded other domains and typical (P1-P1) interjudge consensus. 
NETWORK APPROACH TO REPUTATIONS

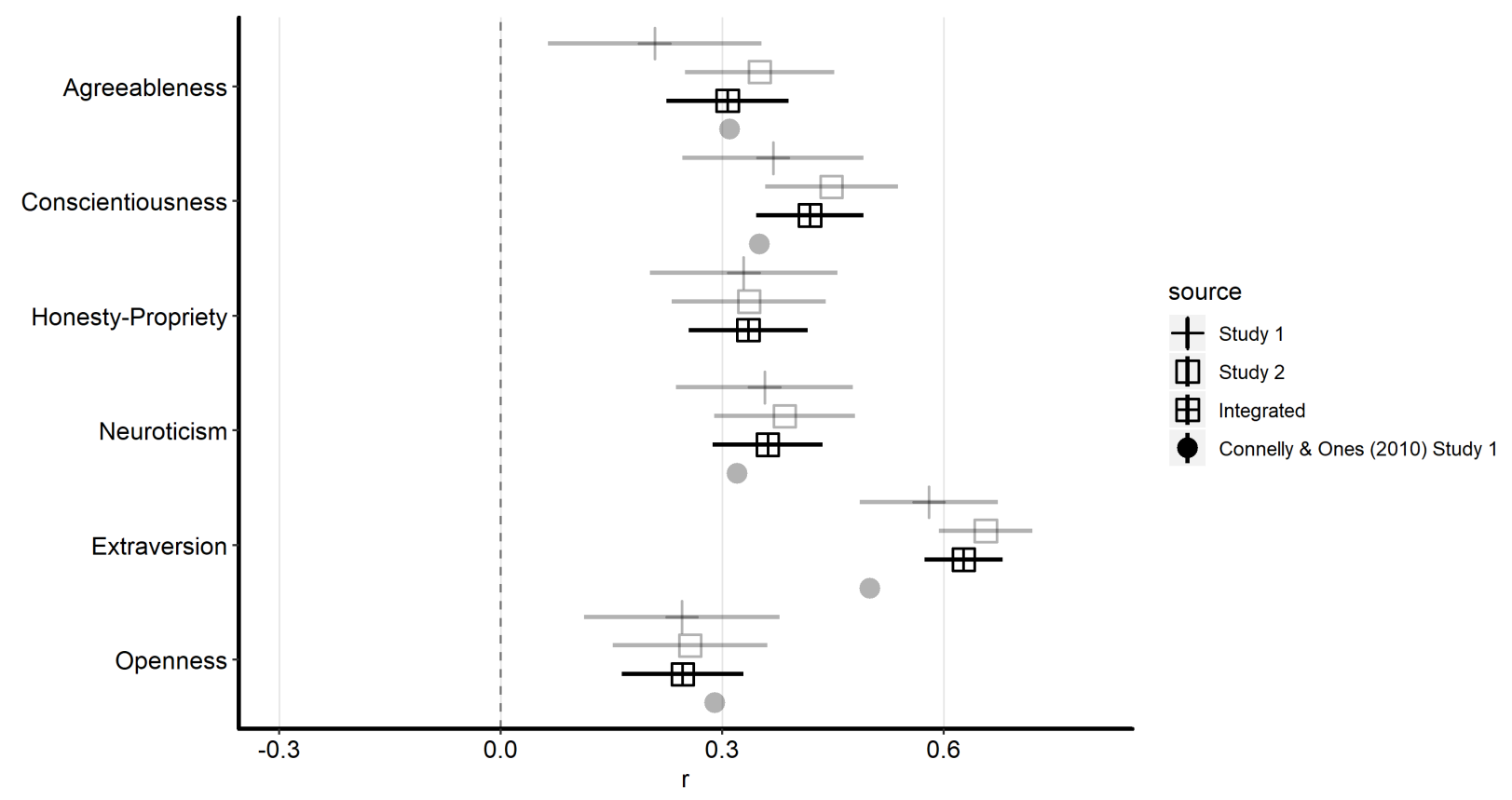

Figure 3. Study 1, Study 2, and integrated estimates of hearsay consensus for the Big Six. Study 1 (box) estimates are based just on the Study 1 sample; Study 2 (cross) estimates are based just on the Study 2 sample (collapsed across conditions); integrated estimates (crossed-box) are based on data from both Studies. Circles again represent meta-analytic estimates of interjudge consensus following naturalistic laboratory interactions, obtained from Study 1 of Connelly \& Ones's (2010) meta-analysis on consensus and self-other agreement.

Domain-wise hearsay accuracy. Hearsay accuracy was assessed for each domain by correlating P2s' ratings of the targets with the targets' self-reports. It speaks to the extent to which the information reaching second-degree perceivers is valid or accurate for each Big Six domain. Figure 4 contains the integrated estimates of hearsay accuracy for the Big Six alongside each study's (non-integrated) estimate and a meta-analytic benchmark of self-other agreement among previously unacquainted participants (Connelly \& Ones, 2010).

As seen in Figure 4, hearsay accuracy was low and indistinguishable from chance for agreeableness, $r=.09,95 \% \mathrm{CI}[-.03, .21], p=.151$. It was moderately low but significantly better than chance for conscientiousness, $r=.16,95 \% \mathrm{CI}[.04, .27], p=.007$. It was low and indistinguishable from chance for honesty-propriety, $r=.06,95 \%$ CI $[-.07, .18], p=.372$. It was moderately low but significantly better than chance for neuroticism, $r=.15,95 \%$ CI $[.03, .26], p$ 
NETWORK APPROACH TO REPUTATIONS

$=.012$. It was moderately high and significantly better than chance for extraversion, $r=.31,95 \%$

CI $[.21, .41], p<.001$, and openness $r=.24,95 \%$ CI [.12, .35], $p<.001$.

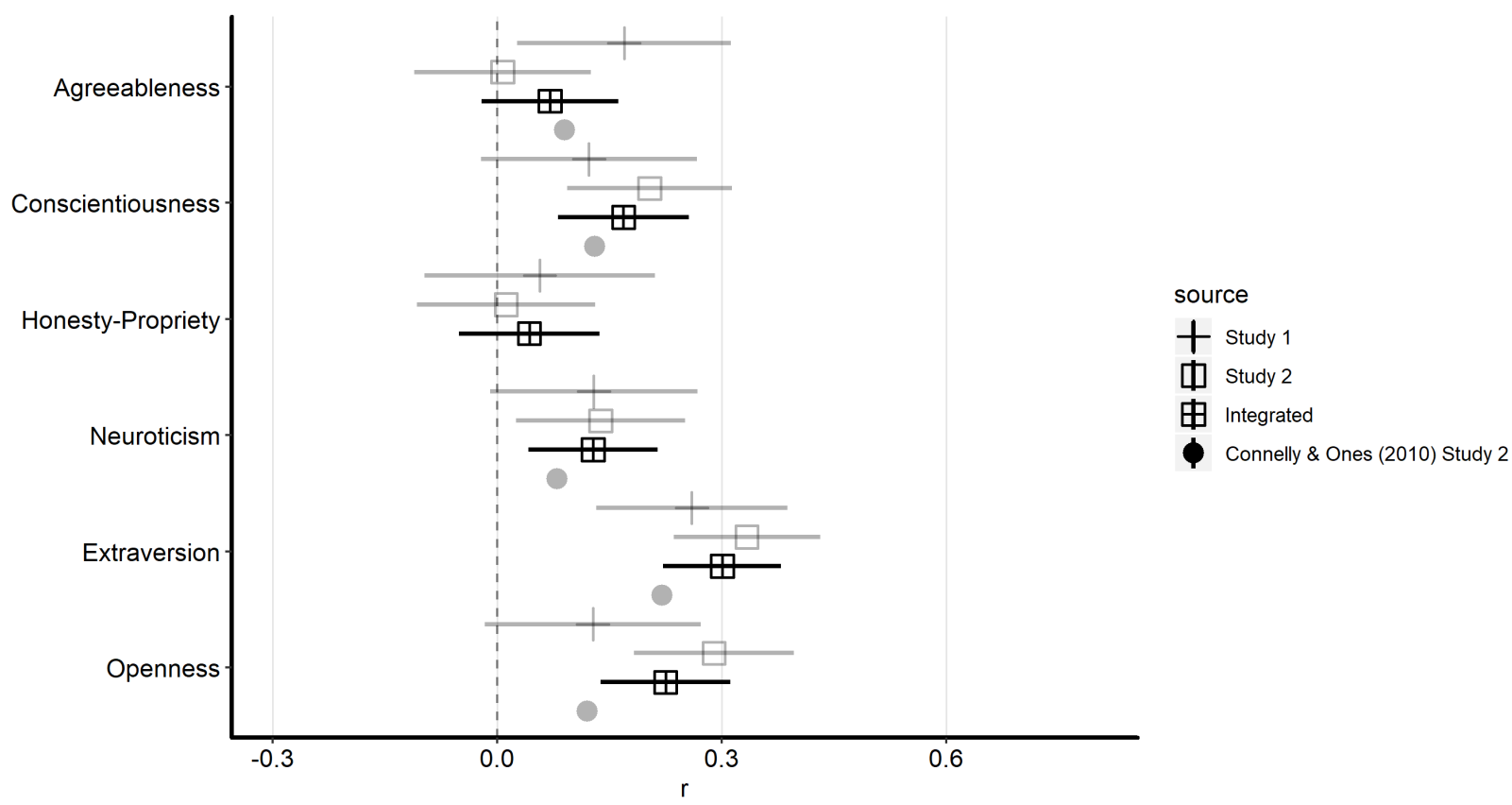

Figure 4. Study 1, Study 2, and integrated estimates of hearsay accuracy for the Big Six. Study 1 (box) estimates are based just on the Study 1 sample; Study 2 (cross) estimates are based just on the Study 2 sample (ignoring condition); integrated estimates (crossed-box) are based on data from both Studies. Circles represent meta-analytic estimates of self-other agreement among previously unacquainted participants, obtained from Study 2 of Connelly \& Ones's (2010) metaanalysis on consensus and self-other agreement (See rows labelled "Stranger" in Table 5 in Connelly \& Ones, 2010).

Descriptively, Figure 4 shows variability across Big Six domains. Hearsay accuracy was relatively the highest for extraversion and openness, in the middle for conscientiousness and neuroticism, and low and indistinguishable from zero for agreeableness and honesty-propriety. CIs for hearsay accuracy overlapped with meta-analytic benchmarks of self-other agreement from direct reputations, suggesting we also cannot rule out the possibility that P2s reach similar degrees of accuracy for each domain as meta-analytic estimates suggest P1s do.

Domain-wise direct accuracy. Domain-wise direct accuracy (i.e., self-P1 agreement) was highly consistent across studies; the integrated estimates were similar to meta-analytic 
NETWORK APPROACH TO REPUTATIONS

estimates (Connelly \& Ones, 2010), but tended to be a little bit higher in our study (see

Supplemental Figure S1).

\section{Individual Difference Moderators}

We used the extended SAM to assess the extent to which triad members' extraversion and P2s' empathy moderated normative consensus and accuracy, distinctive consensus and accuracy, and positivity bias. Individual difference moderators were modeled by including them as level-2 predictors of the intercept and slopes in the SAM. Results are summarized below, and fixed effects from these 14 models are available in Supplemental Table S7 - S20 (see Tables S21 - S34 for Random Effects).

Extraversion. We started by examining the extent to which targets', P1s', and P2s' extraversion moderated hearsay consensus. Target extraversion was associated with slightly lower normative consensus (unstandardized slope $=-0.11,95 \%$ CI $[-0.21,-0.01]$, standardized $=$ $-.05, \mathrm{p}=.035)$. It had small, non-significant impacts on distinctive consensus and positivity (|unstandardized slopes $\mid \leq 0.07 ; p$ 's $>.05$ ). P1 extraversion was associated with lower normative consensus (unstandardized slope $=-0.20,95 \%$ CI $[-0.30,-0.09]$, standardized $=-.09, p<.001$ ) and greater positivity bias (unstandardized slope $=0.17,95 \%$ CI $[0.09,0.26]$, standardized $=.14$, $\mathrm{p}<.001$ ), but it had no impact on distinctive consensus (unstandardized slope $=0.02,95 \%$ CI $[-$ $0.01,0.06]$, standardized $=.02, \mathrm{p}=.185) . \mathrm{P} 2$ extraversion had a similar but slightly stronger impact; it was associated with less normative consensus (unstandardized slope $=-0.36,95 \%$ CI [$0.44,-0.28]$, standardized $=-.18, \mathrm{p}<.001$ ) and greater positivity bias (unstandardized slope $=$ $0.37,95 \%$ CI $[0.32,0.42]$, standardized $=.31, \mathrm{p}<.001)$, but it had no impact on distinctive consensus (unstandardized slope $=-0.01,95 \%$ CI $[-0.04,0.02]$, standardized $=.00, \mathrm{p}=.661$ ). 
NETWORK APPROACH TO REPUTATIONS

We then examined the extent to which targets', P1s', and P2s' extraversion moderated hearsay accuracy. Target extraversion had no effect on any hearsay accuracy component (|unstandardized slopes $\mid \leq 0.01 ; p$ 's $>.05) . \mathrm{P} 1$ extraversion was associated with greater positivity bias (unstandardized slope $=0.11,95 \%$ CI $[0.01,0.20]$, standardized $=.08, \mathrm{p}=.029$ ), and P2 extraversion was associated with less normative accuracy (unstandardized slope $=-0.08,95 \% \mathrm{CI}$ $[-0.13,-0.02]$, standardized $=-.04, p=.007)$ and greater positivity bias (unstandardized slope $=$ $0.19,95 \%$ CI $[0.13,0.26]$, standardized $=.16, \mathrm{p}<.001)$.

To summarize, in triads with more extraverted targets, there was less normative hearsay consensus. In triads with more extraverted perceivers (either P1 or P2), P2s showed greater positivity bias than P1s. P2s' own extraversion was associated with less normative accuracy.

Empathy. We next examined the extent to which P2s' empathy moderated hearsay consensus. There were no significant moderation effects ( $\mid$ unstandardized slopes $\mid \leq 0.10 ; p$ 's $>$ $.05)$, suggesting that empathic concern, fantasy, personal distress, and perspective taking do not affect normative consensus, distinctive consensus, or positivity bias.

We next examined the extent to which P2s' empathy moderated hearsay accuracy. As with hearsay consensus, all empathy moderation effects were non-significant (|unstandardized slopes $\mid \leq .07 ; p$ 's $>.05)$, suggesting that empathic concern, fantasy, personal distress, and perspective taking do not affect normative accuracy, distinctive accuracy, or positivity bias.

\section{Does the Context of Hearsay Moderate Consensus and Accuracy?}

Four hypotheses concerned differences in hearsay consensus and accuracy across the randomly assigned experimental groups in Study 2. As preregistered, we modeled these differences using a multi-group path analytic framework, building on our general approach to domain-wise correlations discussed above. Specifically, we first modelled the data such that 
NETWORK APPROACH TO REPUTATIONS

estimates could differ across conditions, and then assessed the decrement in fit incurred by constraining estimates hypothesized to differ (e.g., hearsay consensus for extraversion in the status and control conditions) to be equal to one another. Our hypotheses would be supported if such equality constraints worsened model fit (indicated by a significant $\chi^{2}$ test, a positive $\Delta_{\text {AIC }}$, and a positive $\left.\Delta_{\mathrm{BIC}}\right)$.

Status-relevant context and extraversion. Our first hypothesis was that hearsay consensus for extraversion would be greater in the status condition than in the control condition. We tested this by first estimating a model where hearsay consensus for extraversion could differ across conditions, then a model where hearsay consensus was equal in the status and control conditions. The hypothesis would be supported if the latter fit the data worse (as determined by a significant $\chi_{\mathrm{d}}^{2}$ and positive $\Delta_{\mathrm{AIC}}$ and $\Delta_{\mathrm{BIC}}$ values) and the results showed greater hearsay consensus for extraversion in the status condition.

Figure 5 displays hearsay consensus, hearsay accuracy, and direct accuracy for extraversion across condition. As seen in Figure 5, descriptively, hearsay consensus for extraversion was slightly higher in the status condition $(r=.68 ; 95 \% \mathrm{CI}[.54, .82])$ than the control condition $(r=.57 ; 95 \%$ CI $[.41, .74])$. However, the model of no difference did not fit the data significantly worse $\left(\Delta_{\mathrm{AIC}}=-0.65 ; \Delta_{\mathrm{BIC}}=-3.29 ; \chi_{\mathrm{d}}^{2}(1)=1.35, p=.244\right)$. The negative $\Delta_{\mathrm{AIC}}$, $\Delta_{\mathrm{BIC}}$, and non-significant $\chi^{2} \mathrm{~d}$ all suggest that hearsay consensus for extraversion does not differ across condition. 
NETWORK APPROACH TO REPUTATIONS

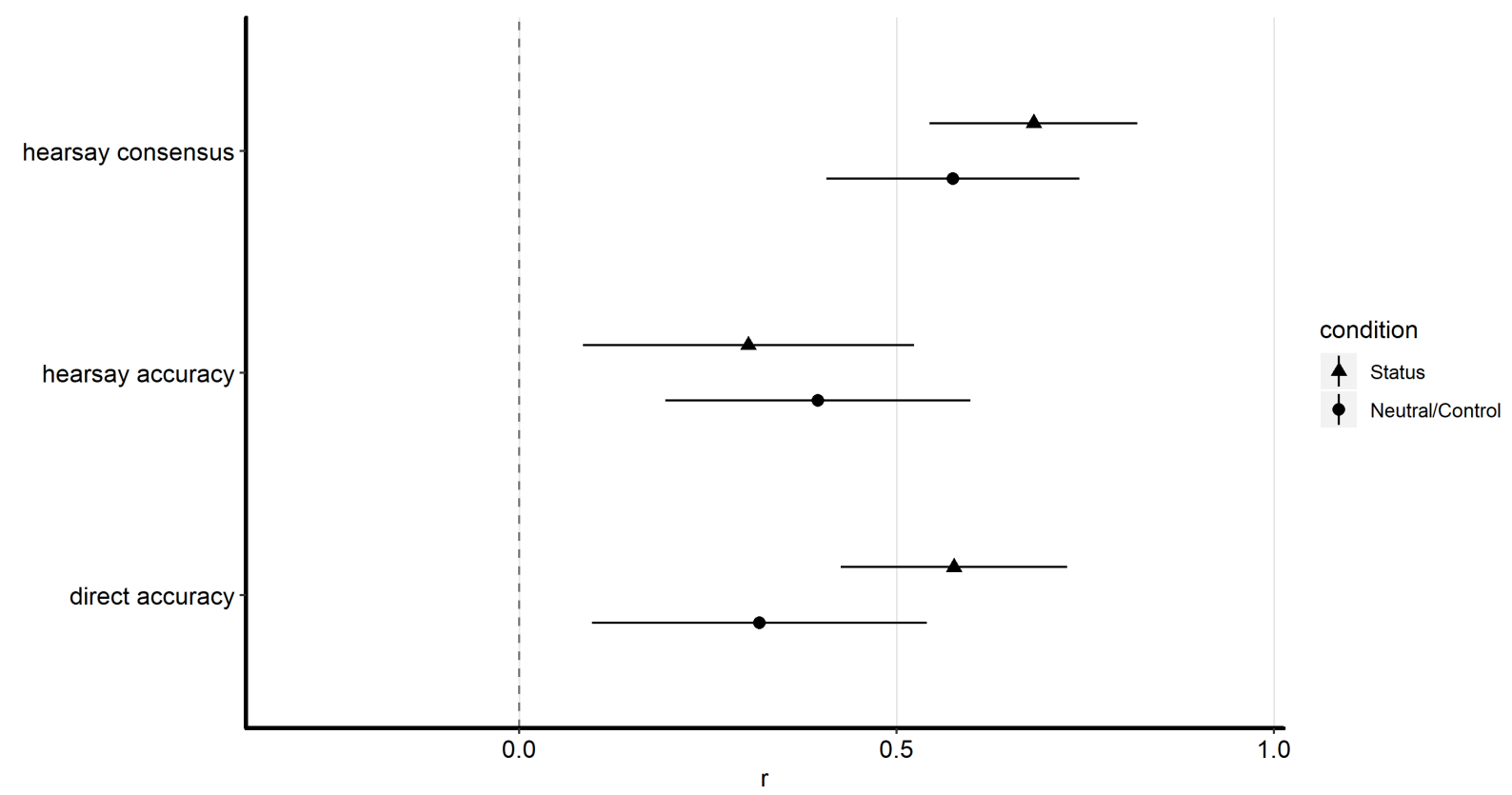

Figure 5. Hearsay consensus, accuracy, and direct accuracy for extraversion across Status and Neutral/Control conditions. Shapes represent point estimates and lines correspond to 95\% CI's around those estimates.

Our second hypothesis predicted greater hearsay accuracy for extraversion in the status condition, and we tested it similarly. This hypothesis was also not supported. Descriptively, hearsay accuracy for extraversion was slightly lower in the status condition $(r=.30,95 \% \mathrm{CI}$ $[.08, .52])$ than the control condition $(r=.40,95 \%$ CI $[.19, .60])$. The model consistent with no difference did not fit the data worse according to $\chi^{2}{ }_{\mathrm{d}}$ and fit better according to $\Delta_{\mathrm{AIC}}$ and $\Delta_{\mathrm{BIC}}$ $\left(\Delta_{\mathrm{AIC}}=-1.88 ; \Delta_{\mathrm{BIC}}=-4.52 ; \chi^{2}{ }_{\mathrm{d}}(1)=0.12, p=.727\right)$.

We thus found evidence inconsistent with the hypothesis that the status manipulation would affect the consistency or accuracy of hearsay reputations of extraversion. Follow-up analyses with direct accuracy for extraversion (the third parameter displayed in Figure 5) similarly showed no differences between the status and control conditions (see Supplemental Table S35 for model comparison results). 
NETWORK APPROACH TO REPUTATIONS

Affiliation-relevant context and agreeableness. We hypothesized that hearsay

consensus for agreeableness would be greater in the affiliation condition than the control condition, which we tested similarly to the hypotheses for the status manipulation and extraversion. Figure 6 contains the estimates for hearsay consensus, hearsay accuracy, and direct accuracy for agreeableness across condition. Descriptively, we found a slightly lower estimate of hearsay consensus for agreeableness in the affiliation condition $(r=.34,95 \% \mathrm{CI}[.10, .58])$ than the control condition $(r=.41,95 \%$ CI $[.19, .62])$. The model comparison results were consistent with no difference across these conditions $\left(\Delta_{\mathrm{AIC}}=-1.89 ; \Delta_{\mathrm{BIC}}=-4.54 ; \chi^{2}{ }_{\mathrm{d}}(1)=0.11, p=.741\right)$. The hypothesis that hearsay accuracy for agreeableness would be greater in the affiliation condition was also not supported. Hearsay accuracy was descriptively higher in the affiliation condition $(r=.26,95 \% \mathrm{CI}[.00, .53])$ than the control condition $(r=.03,95 \% \mathrm{CI}[--.26, .32])$ condition, but the model fit evidence was inconsistent with a difference $\left(\Delta_{\mathrm{AIC}}=-1.06 ; \Delta_{\mathrm{BIC}}=-\right.$ $\left.3.71 ; \chi^{2}(1)=0.94, p=.333\right)$.

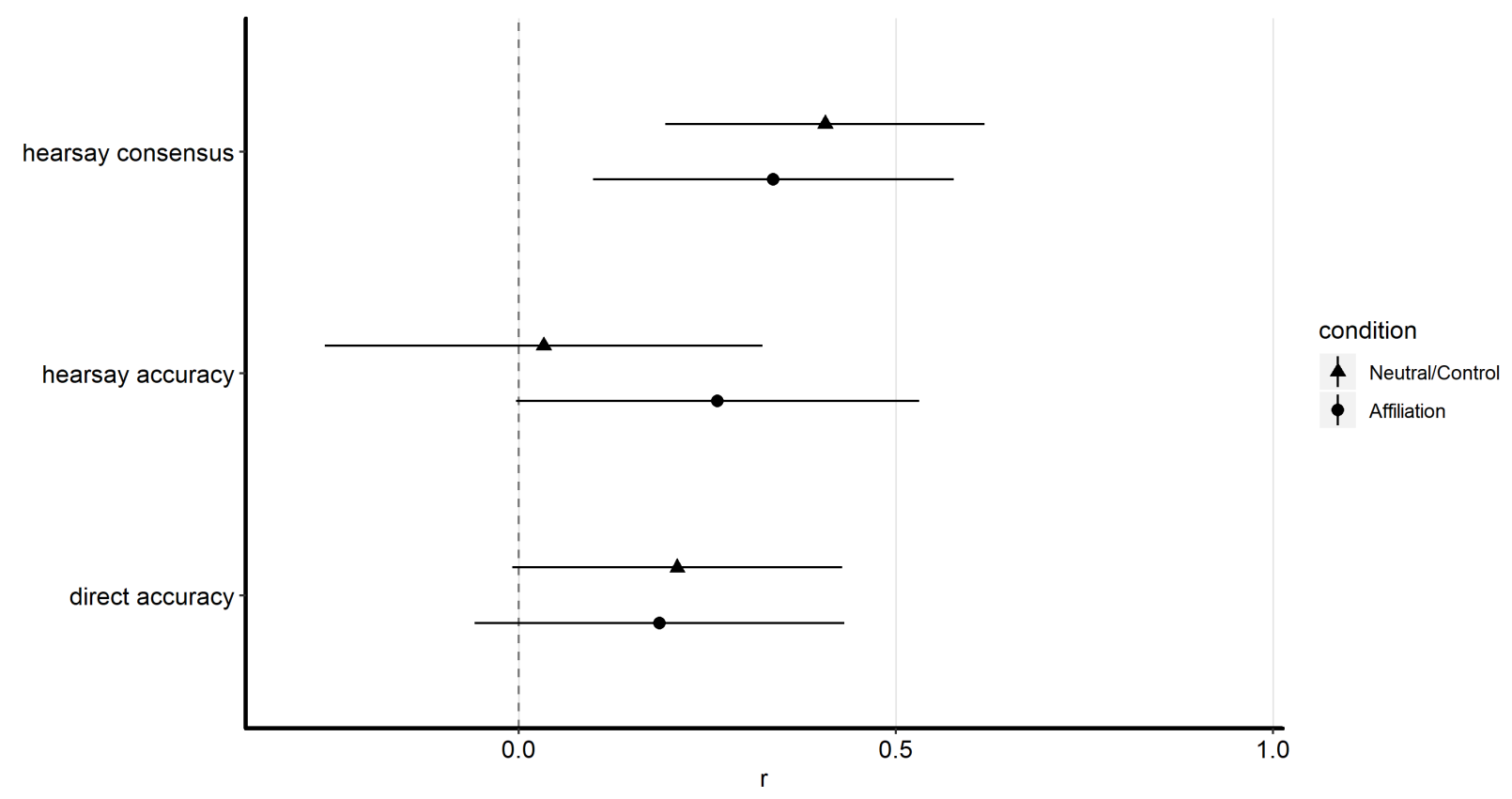

Figure 6. Hearsay consensus, accuracy, and direct accuracy for agreeableness across conditions. Shapes represent point estimates and lines correspond to 95\% CI's around those estimates. 
NETWORK APPROACH TO REPUTATIONS

Thus, there was virtually no support for the hypothesis that the affiliation manipulation would increase hearsay consensus or accuracy for agreeableness. Follow-up analyses with direct accuracy (see Figure 6) similarly demonstrated no effect of the manipulation (see Supplemental Table S36 for model comparison results).

Effects of reputation on leadership judgments. We hypothesized that information about targets' extraversion flowing from P1s to P2s would affect P2s' perceptions of targets' leadership potential. We tested this hypothesis in a mediation model that mirrored these predictions. Figure 7a shows this mediation model with the corresponding (unstandardized) regression slopes, 95\% CIs, and $p$ values. As seen in Figure 7a, we found support for the hypothesized relations: P1s' perceptions of targets' extraversion were shared with P2s ( $b=0.74$, 95\% CI [0.60, 0.86], $p<.001$ ), which in turn predicted P2s' perceptions of targets' leadership potential $(b=0.77,95 \% \mathrm{CI}[0.47,1.07], p<.001)$. The mediated (indirect) effect was significantly different from zero, moderately large in magnitude, and consistent with a moderate to large effect $\left(b_{\text {indirect }}=0.56,95 \% \mathrm{CI}[0.35,0.80], p<.001\right)$. In effect, this means that each scale point increase in P1s' perceptions of targets' extraversion is related to roughly half a scale point increase in P2s' perceptions of targets' leadership potential (with the 95\% confidence interval ranging from one-third to four-fifths of a scale point increase), which appeared to be transmitted through P2s' perceptions of targets' extraversion.

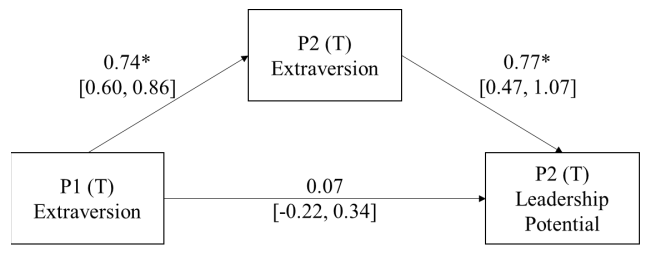

(a) Extraversion and leadership judgments.

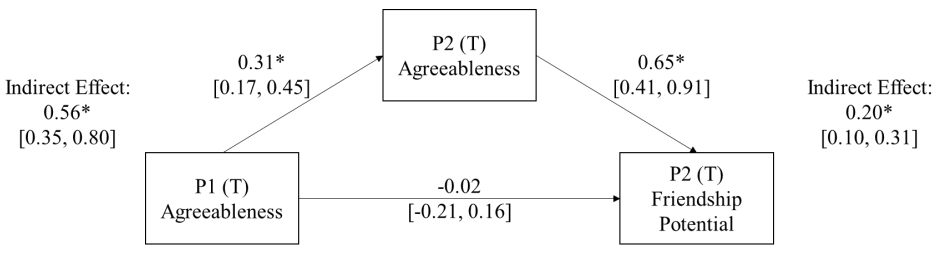

(b) Agreeableness and friendship judgments.

Figures 7a-b. Mediation models. Standard errors were obtained via bootstrapping with 1000 resamples. The point estimate and $95 \% \mathrm{CI}$ for the indirect effect are shown to the right. ${ }^{*} \mathrm{p}<.001$ 
NETWORK APPROACH TO REPUTATIONS

We next investigated the specificity of the extraversion-leadership connection and assess the possibility that the observed effect is driven by global positive evaluation, in a nonpreregistered follow-up analysis. We did so by regressing P2s' perceptions of targets' leadership potential on indirect effects for all of the Big Six in a multiple mediation model. A graphical depiction of this model is shown in Supplemental Figure S2. In this model, the indirect effect of extraversion judgments remained significant, but did drop in magnitude $\left(b_{\text {indirect }}=0.36,95 \% \mathrm{CI}\right.$ $[0.16,0.58]), p=.001)$, now indicating a moderately small indirect effect and consistent with a small to moderate effect. Additionally, two non-hypothesized indirect effects emerged as significant. P1s' perceptions of the targets' conscientiousness were shared with P2s $(b=0.43$, 95\% CI [0.28, 0.59], $p<.001)$, which in turn affected P2s' perceptions of targets' leadership potential $\left(b=0.60,95 \% \mathrm{CI}[0.23,0.92], p=.001 ; b_{\text {indirect }}=0.26,95 \% \mathrm{CI}[0.08,0.46], p=.009\right)$. Likewise, P1s' perceptions of targets' openness were shared with P2s ( $b=0.19,95 \%$ CI $[0.08$, $0.30], p<.001)$, which in turn affected their perceptions of targets' leadership potential $(b=$ $\left.0.61,95 \% \mathrm{CI}[0.24,0.96], p=.002 ; b_{\text {indirect }}=0.12,95 \% \mathrm{CI}[0.03,0.22], p=.016\right)$. Note that each of these indirect effects was smaller than the hypothesized effect, and each were consistent with negligible effect sizes (CIs ranged below 0.10). Taken together P1s' perceptions of targets' extraversion, conscientiousness, and openness influence P2s' perceptions of the targets' leadership potential such that a unit increase in each is expected to increase P2s' perceptions of targets' leadership potential by roughly one-third, one-fourth, and one-tenth of a likert-scale unit respectively.

Effects of reputation on friendship judgments. We also hypothesized that information about targets' agreeableness flowing from P1s to P2s would affect P2s' perceptions of targets' friendship potential. We tested this hypothesis in a mediation model. This model and the 
NETWORK APPROACH TO REPUTATIONS

corresponding unstandardized regression slopes, 95\% CIs, and $p$ values are shown in Figure $7 \mathrm{~b}$.

Consistent with the hypothesis, P1s shared information about targets' agreeableness with P2s ( $b$

$=0.31,95 \% \mathrm{CI}[0.17,0.45], p<.001)$, which in turn affected P2s' perceptions of targets'

friendship potential $\left(b=0.65,95 \%\right.$ CI $[0.41,0.91], p<.001 ; b_{\text {indirect }}=0.20,95 \%$ CI $[0.10,0.32]$,

$p<.001$; see Figure 7b). The mediated (indirect) effect was significant and consistent with a

small or moderate effect size. This means that each scale point increase in P1s' perceptions of a

targets' agreeableness is associated with an increase of about one fifth of a scale point in P2s'

perceptions of the targets' friendship potential, and this is achieved primarily via effecting P2s'

perceptions of the targets' agreeableness.

Mirroring our approach for leadership potential, we conducted a non-pre-registered

follow-up analysis aimed at assessing the specificity of the link between perceptions of agreeableness and friendship potential by modelling hearsay reputations of friendship potential as a function of indirect effects of direct and hearsay reputations of each of the Big Six; this model is depicted in Supplemental Figure S3. The indirect effect of agreeableness dropped slightly but remained significant $\left(b_{\text {indirect }}=0.15,95 \%\right.$ CI $\left.[0.05,0.27], p=.008\right)$. The indirect effects for the remaining Big Six were small and not significant (all $\left|b_{\text {indirect|}}\right|$ 's $<.05$; all $p$ 's $>.1$ ). Thus, it appears that hearsay reputations of friendship potential were, within the Big Six, mostly a function of hearsay reputations (and indirectly, direct reputations) of agreeableness.

\section{General Discussion}

We used a network approach to study how reputation information flows across networks of perceivers in a naturalistic laboratory setting. Second-degree perceivers, who only heard about a target through hearsay, agreed with first-degree perceivers about the personalities of targets. Second-degree perceivers also achieved modest levels of normative and distinctive accuracy, as 
NETWORK APPROACH TO REPUTATIONS

evidenced by agreement with targets' self-reports - but this agreement was lower than hearsay consensus and lower than direct accuracy. Contrary to predictions based on previous work, there was no evidence that the extraversion of anyone in the network led to better accuracy; instead, extraversion reduced normative hearsay consensus and accuracy and increased evaluative bias. An experimental manipulation of participants' conversational goals had little to no impact on domain-wise hearsay consensus or accuracy for relevant personality domains. We found that the transmission of reputational knowledge had consequences for important social judgments about whether targets would be good leaders and friends. We believe these results demonstrate the usefulness of the network approach to reputation, but they represent merely a first step in a broader research program.

\section{Studying the Flow of Reputational Information}

The network approach to reputations conceptualizes reputations as information flowing across a network of perceivers. By comparing the information stored in the nodes of that network - that is, the impressions formed by perceivers at different distances from a target - we can draw inferences about the valence, strength, and validity of information flow between people. The present findings have implications for each.

Hearsay consensus and accuracy. The hearsay consensus results showed substantial agreement between perceivers in different layers of the network. This was most pronounced for impressions of extraversion, where hearsay consensus exceeded meta-analytic estimates of agreement among first-degree perceivers, though there was substantial consensus for all trait domains. Hearsay thus appeared to contain a strong and consistent signal. This signal, however, appeared only somewhat valid, as shown by the more modest estimates of hearsay accuracy. Indeed, consistent with prior work demonstrating that normative accuracy increases with greater 
NETWORK APPROACH TO REPUTATIONS

interpersonal knowledge (Wessels et al., 2020), we found significantly less normative accuracy among second-degree perceivers than among first-degree perceivers. Likewise, distinctive accuracy was also significantly lower for P2s than for P1s. Together, this suggests that the hearsay information flowing from $\mathrm{P} 1$ to $\mathrm{P} 2$ is less valid than the information flowing through direct observation from $\mathrm{T}$ to $\mathrm{P} 1$. The latter has been the primary focus of prior research on interpersonal perception. In many studies it is moderately valid (see Connelly \& Ones, 2010), and if anything, the estimates of direct accuracy were higher than usual in this study. So why did we find so little hearsay accuracy?

One possibility is that most of the signal loss occurs between $\mathrm{T}$ and $\mathrm{P} 1$, but that P1s are then reasonably effective at sharing almost all of the valid information they have with P2s. The signal loss may occur from $\mathrm{T}$ to $\mathrm{P} 1$ because $\mathrm{P} 1 \mathrm{~s}$ have to integrate two sources of information, targets' verbal self-descriptions and their behavior, which may sometimes conflict. By contrast, P2s have just a single (seemingly consistent) source of information in P1s' descriptions of Ts. This would be broadly consistent with our results. This is also consistent with prior work demonstrating that P1-P1 consensus generally exceeds self-P1 agreement (Connelly \& Ones, 2010), and with theoretical models emphasizing the unique perspective of the self (Vazire, 2010).

A second, potentially complementary possibility draws upon insights from Kenny’s (2004) PERSON model of interpersonal perception. PERSON posits that impressions are composed of multiple components. Two components, stereotypes and personality, are consistent and shared by all perceivers. However, perceivers also develop stable but idiosyncratic beliefs about targets, labeled the opinion component. Opinion can make up a large portion of a perceiver's overall impression. When two different perceivers form impressions by observing a 
NETWORK APPROACH TO REPUTATIONS

target independently, their opinion components will be uncorrelated. However, when

impressions are transmitted by perceivers through gossip, idiosyncratic opinions will be part of

what they pass along. This would lead to a strong, consistent, but substantially invalid, signal

being communicated from P1s to P2s, and it would result in higher agreement than is typically

observed in dyadic consensus studies.

This has implications for how reputations might spread in larger and more complex networks. The stereotype and personality components originate from a target and therefore would spread through the entire network, producing global consistency among all nodes. By contrast, an opinion component originates with one perceiver, and therefore would spread only to other nodes who received information from that perceiver, producing local consistency in just a region of a network. This possibility, derived from the PERSON model and suggested by the present data, could be further studied in experimental networks with multiple second-degree perceivers, in naturally occurring real-world networks, or in modeling and simulation studies.

Why were hearsay consensus and accuracy higher for extraversion than other domains?

Previous research has suggested that the observability of trait domains affects first-degree perceivers' accuracy (Vazire, 2010). Moreover, conversational norms may guide people to share information about domains they feel they have some knowledge about (Grice, 1975). This may further winnow the domains of reputation information that are shared with second-degree perceivers and beyond.

Positivity bias. Were second-degree perceivers more or less positive than first-degree perceivers? We examined this question in two ways. In the consensus analysis, the positivity of different traits did not predict $\mathrm{P} 2 \mathrm{~s}^{\prime}$ ratings after controlling for P1s' ratings. In the accuracy analysis, we tested whether P2s and P1s differed in their positivity after controlling for targets' 
NETWORK APPROACH TO REPUTATIONS

self-reports; the difference was small in magnitude and significant, though barely under the threshold. On the whole, there may have been a small difference in positivity but not a sizeable one.

These results potentially contradict Wessels and colleagues (2020) interpretation that people substitute their lack of information about targets with positivity. P2s never met the targets and presumably had less information, but if anything they demonstrated less positivity bias, not more. This small decreases in positivity could reflect positionality (first versus second degree relationships) rather than interpersonal knowledge per se. It may be the case that interacting with someone directly leads to more positive feelings and ultimately a more positive impression than simply hearing about them, even when hearing about them from someone who has a positively biased impression. Future work could further tease apart effects of acquaintanceship and positionality on positivity by varying how well P1s get to know targets, and how much opportunity they have to convey information to P2s.

\section{What Moderates the Flow of Reputational Information?}

We hypothesized that the extraversion levels of members of the network would increase consensus and accuracy. Extraversion is associated with greater talkativeness and expressivity, which has been associated with accuracy in previous research (Funder, 1995; Human \& Biesanz, 2013). Extraversion could increase the amount of information flowing to and from nodes in the network. The results, however, showed the opposite: when extraversion had a moderating effect, it was in the direction of less normative accuracy and greater positivity bias. This was more the case with perceivers' extraversion (P1 and P2) than targets' extraversion. What might explain these unexpected findings, if they are be replicable? Extraversion is associated with positive affect, so speculatively, that may have been transferred by association to the targets that they 
NETWORK APPROACH TO REPUTATIONS

were discussing. It is harder to say why normative consensus and accuracy would be lower when the perceivers were higher in extraversion.

In Study 2 we used an experimental manipulation to focus the hearsay exchange on specific aspects of the target - the extent to which they would make a good leader or friend. The manipulation had little impact on the resulting impressions. There are several possible explanations for this finding. First, it may be that relevancy has little or no impact on hearsay; perhaps people share information they have about a target person regardless of how relevant that information is to $\mathrm{P} 2$. We believe this broad conclusion is unlikely, and more work would be needed to make a strong case that relevancy has no impact on reputational discourse.

Another possibility is that the instruction manipulation used in Study 2 may have been too weak. Previous work has demonstrated an effect of subtle instruction manipulations on direct accuracy (Biesanz \& Human, 2010), but perhaps a stronger manipulation is needed to affect hearsay consensus or accuracy. Future researchers could consider increasing the incentive of a consistent or accurate impression, perhaps by having some perceived consequence or valued outcome for P1s or P2s.

Despite limited success in identifying moderators in the present studies, we believe that this is a promising direction for future theorizing and research. People - the nodes in reputation networks - are not all the same or interchangeable. Likewise, relationships - the edges - are not the same either. In order to get traction on this study's research questions, we held many things constant that would vary in real-world networks. Networks can vary in tie strength (such as acquaintanceship or liking). They can vary in multiplexity, meaning that people have multiple kinds of relationships (such as kinship, friendships, or work). Additionally, the goals that network members have when they gossip, and the contexts in which they interact, are likely to 
NETWORK APPROACH TO REPUTATIONS

affect both the amount and kind of reputational information that people spread. The network approach to reputations affords the theoretical, methodological, and analytic foundation to begin posing and answering such questions.

\section{Social Consequences of Hearsay Reputations}

In Study 2, we found that reputations affected social decisions that hearsay perceivers made about the target. Analyses showed that information flowed from P1s to P2s about targets' standing on extraversion and agreeableness. This information was associated with P2s' perceptions of targets' leadership and friendship potential respectively. We additionally found that information about targets' standing on conscientiousness and openness likewise flowed from P1 to P2 and was associated with P2s' perception of targets' leadership potential. These results demonstrate that hearsay reputations not only include information about targets' personalities but additionally provide $\mathrm{P} 2 \mathrm{~s}$ with information they might use in deciding if and how to interact with the target person(s). Thus, reputations can influence the opportunities people are afforded in love and work.

This finding fits more generally with functionalist accounts of gossip and reputation. When people socialize, a large proportion of what they do is gossip (Dunbar, Marriott, \& Duncan, 2007). When people have to make important decisions about one another - who to trust, who to pursue relationships with, who to work with - reputations can be valuable to decisionmakers by giving them more information than what they have directly observed themselves. For this reason, gossip may have evolved as a behavior to help humans solve some of the problems of coordinating behavior in large groups (Dunbar, 2010; Feinberg, Willer, \& Schultz, 2014). At the same time, some of the findings from the present study, and implications drawn from them, suggest ways that the consequences of reputations can go awry. For example, the finding that 
NETWORK APPROACH TO REPUTATIONS

consensus was stronger than accuracy suggests that at least some of the time, groups might arrive at a stable view of someone's personality that is wrong. If they act on such a consensus, that can have serious effects on the target's standing in the group and their well-being.

Of course, that finding came from a study of how reputations spread among new acquaintances. It is possible that longer relationships embedded in more complex networks might provide more opportunities for updating and correction; at the same time, they may offer more ways for inaccurate reputations to become consolidated and resistant to change. Answering these questions will probably require combining the present approach, which offers an up-close look at information flow one edge at a time, with other methods for examining how these processes scale up in larger and more complex kinds of networks.

\section{Limitations \& Future Directions}

Several limitations are worth considering when interpreting the present findings. First is the issue of accuracy. As we note in the introduction, there is no perfect accuracy criterion (see Funder, 1995; Kenny, 1994; Robins \& John, 1997), and often it is recommended to use several criteria to evaluate accuracy (Funder, 1995). We used targets' self-reports in the present study, which is a common but imperfect accuracy criterion in interpersonal perception research. One important direction for future work is to examine hearsay accuracy with a more comprehensive set of accuracy criteria.

Another limitation is the nature of the T-P1 interaction and its impact on the present results. The T-P1 interaction consisted primarily of each describing themselves to one another, and P1s perception may have been largely based on what targets said about themselves, which could have inflated our estimates of direct and hearsay accuracy (Borkenau, Leising, \& Fritz, 2014). Of course, perceivers did not have to believe targets, and may have used behavioral cues 
NETWORK APPROACH TO REPUTATIONS

available to them in this interaction (e.g., how expressive targets were as they answered questions, how nervous they appeared, laughter, etc.). Indeed, like many face-to-face interactions, there was likely plenty of behavioral evidence of extraversion, but perhaps less behavioral evidence (and more self-descriptive evidence) of other domains. The extent to which these results generalize to situations in which P1s have more behavioral cues should be examined in future work.

Additionally, we had only one perceiver per position in the reputational network, and so we had only one observation of direct and hearsay reputation information per target. In this way, the present design is more of an extension of the dyadic paradigm than the larger perceiver-target block or round-robin designs common in interpersonal perception (Kenny, 1994). Larger designs would afford greater precision and new analytic possibilities that will be important in expanding and elaborating the network approach to reputations.

Finally, it is worth considering the possibility that P2s saw targets either when arriving to the lab or when they were being directed between rooms (e.g., during the transition from Phase 1 to 2 of the procedure), and that such brief, visual information contributed to their impressions of the targets (Ambady \& Rosenthal, 1992; Borkenau et al., 2009). We think that this happened rarely, if at all. We designed the procedure to minimize this possibility by scheduling participants to arrive in different areas of the building, and then escorting them separately to the lab. Moreover, if a participant in the $\mathrm{P} 2$ role were to see the corresponding target outside the lab rooms, they would later have to infer that that was the person they were told about. However, since we did not ask about this during the debriefing, we cannot rule it out. If participants managed to see each other and make the connection, previous research suggests that brief visual 
NETWORK APPROACH TO REPUTATIONS

information contributes modestly to accuracy in extraversion and conscientiousness (Borkenau et al., 2009).

\section{Conclusion}

Craik's (2009) network conceptualization, combined with the concepts and tools of interpersonal perception research, offers a powerful new way to theorize about and empirically study reputations. Inspired by that conceptualization, we developed an experimental paradigm and a data-analytic framework to study how reputations form and spread through micronetworks. In two studies, we applied this approach to studying the spread of reputational information about personality among previously-unacquainted participants. But we see these initial studies and results as only a beginning, and we have made our materials and an R package available to facilitate adoption of this paradigm by other researchers. Real-world reputations can contain a wide range of information; and they spread through larger and more complex networks, among perceivers in many different contexts and with many different goals, through people with many different kinds of relationships. It is our hope that other researchers will see the importance and potential of this work, and that they will be as excited as we are to continue it in the future. 
NETWORK APPROACH TO REPUTATIONS

\section{References}

Allport, G. W., \& Odbert, H. S. (1936). Trait-Names: A Psycho-lexical Study. Psychological Monographs, 47, i-171. http://dx.doi.org/10.1037/h0093360

Ambady, N., \& Rosenthal, R. (1993). Half a minute: Predicting teacher evaluations from thin slices of nonverbal behavior and physical attractiveness. Journal of Personality and Social Psychology, 64(3), 431-441. https://doi.org/10.1037/0022-3514.64.3.431

Anderson, C., John, O. P., Keltner, D., \& Kring, A. M. (2001). Who attains social status? Effects of personality and physical attractiveness in social groups. Journal of Personality \& Social Psychology, 81, 116-132. https://doi.org/10.1037//00223514.81.1.116

Aron, A., Melinat, E., Aron, E. N., Vallone, R. D., \& Bator, R. J. (1997). The Experimental Generation of Interpersonal Closeness: A Procedure and Some Preliminary Findings. Personality and Social Psychology Bulletin, 23, 363-377. https://doi.org/10.1177/0146167297234003

Back, M. D., Stopfer, J. M., Vazire, S., Gaddis, S., Schmukle, S. C., Egloff, B., \& Gosling, S. D. (2010). Facebook profiles reflect actual personality, not self-idealization. Psychological Science, 21, 372-374. https://doi.org/10.1177/0956797609360756

Bates, D., Maechler, M., Bolker, B., \& Walker, S. (2015). Fitting Linear Mixed Effects Models Using lme4. Journal of Statistical Software, 67(1), 1-48. https://doi.org/10.18637/jss.v067.i01.

Biesanz, J. C. (2010). The Social Accuracy Model of Interpersonal Perception: Assessing Individual Differences in Perceptive and Expressive Accuracy. Multivariate Behavioral Research, 45, 853-885. https://doi.org/10.1080/00273171.2010.519262

Biesanz, J. C., \& Human, L. J. (2010). The cost of forming more accurate impressions: accuracymotivated perceivers see the personality of others more distinctively but less normatively than perceivers without an explicit goal. Psychological Science, 21, 589-594. https://doi.org/10.1177/0956797610364121

Borkenau, P., Leising, D., \& Fritz, U. (2014). Effects of communication between judges on consensus and accuracy in judgments of people's intelligence. European Journal of Psychological Assessment, 30(4), 274-282. https://doi.org/10.1027/1015-5759/a000188

Borkenau, P., Brecke, S., Möttig, C., \& Paelecke, M. (2009). Extraversion is accurately perceived after a 50-ms exposure to a face. Journal of Research in Personality, 43(4), 703706. https://doi.org/10.1016/j.jrp.2009.03.007 
NETWORK APPROACH TO REPUTATIONS

Buss, D. M. (2011). Personality and the adaptive landscape. In D. M. Buss \& P. H. Hawley (Eds.), The Evolution of Personality and Individual Differences (pp. 29-57).

Buss, D. M. (1996). Social adaptation and five major factors of personality. In J. S. Wiggins (Ed.), The five-factor model of personality: Theoretical Perspectives (pp. 180-207). New York: Guilford Press.

Carlson, E. N., Vazire, S., \& Furr, R. M. (2011). Meta-Insight: Do People Really Know How Others See Them? Journal of Personality \& Social Psychology, 101, 831-846. https://doi.org/10.1037/a0024297

Chaplin, W. F., \& Panter, A. T. (1993). Shared meaning and the convergence among observers' personality descriptions. Journal of Personality, 61, 553-585. https://doi.org/10.1111/j.1467-6494.1993.tb00782.x

Connelly, B. S., \& Ones, D. S. (2010). An other perspective on personality: Meta-analytic integration of observers' accuracy and predictive validity. Psychological Bulletin, 136, 1092-1122. https://doi.org/10.1037/a0021212

Cottrell, C. A., Neuberg, S. L., \& Li, N. P. (2007). What do people desire in others? A sociofunctional perspective on the importance of different valued characteristics. Journal of Personality and Social Psychology, 92, 208-231. https://doi.org/10.1037/0022$\underline{3514.92 .2 .208}$

Craik, K. H. (2009). Reputation: A network interpretation. New York, NY: Oxford University Press.

Cronbach, L. J. (1955). Processes affecting scores on understanding of others and assumed similarity. Psychological Bulletin, 52, 177-193. https://doi.org/10.1037/h0044919

Davis, M. H. (1983). Measuring individual differences in empathy: Evidence for a multidimensional approach. Journal of Personality and Social Psychology, 44, 113-126. http://dx.doi.org/10.1037/0022-3514.44.1.113

Denissen, J. J. a., \& Penke, L. (2008). Motivational individual reaction norms underlying the Five-Factor model of personality: First steps towards a theory-based conceptual framework. Journal of Research in Personality, 42, 1285-1302. https://doi.org/10.1016/j.jrp.2008.04.002

DesJardins, N. M. L., Srivastava, S., Küfner, A. C. P., \& Back, M. D. (2015). Who Attains Status? Similarities and Differences Across Social Contexts. Social Psychological and Personality Science, 6, 692-700. https://doi.org/10.1177/1948550615580171

DeYoung, C. G. (2015). Cybernetic Big Five Theory. Journal of Research in Personality, 56, 33-58. https://doi.org/10.1016/j.jrp.2014.07.004 
NETWORK APPROACH TO REPUTATIONS

Dunbar, R. I. M. (2004). Gossip in evolutionary perspective. Review of General Psychology, 8, 100-110. https://doi.org/10.1037/1089-2680.8.2.100

Dunbar, R. I. M., Marriott, A., \& Duncan, N. D. C. (1997). Human conversational behavior. Human Nature, 8, 231-246. https://doi.org/10.1007/BF02912493

Dunning, D. (1999). A Newer Look: Motivated Social Cognition and the Schematic Representation of Social Concepts. Psychological Inquiry, 10, 1-11. https://doi.org/10.1207/s15327965pli1001_1

Farley, S. D. (2011). Is gossip power? The inverse relationships between gossip, power, and likability. European Journal of Social Psychology, 41, 574-579. https://doi.org/10.1002/ejsp.821

Feinberg, M., Willer, R., \& Schultz, M. (2014). Gossip and Ostracism Promote Cooperation in Groups. Psychological Science, 25, 656-664. https://doi.org/10.1177/0956797613510184

Funder, D. C. (1995). On the accuracy of personality judgment: A realistic approach. Psychological Review, 102, 652-670. https://doi.org/10.1037/0033-295X.102.4.652

Funder, D. C., Kolar, D. C., \& Blackman, M. C. (1995). Agreement among judges of personality: interpersonal relations, similarity, and acquaintanceship. Journal of Personality and Social Psychology, 69, 656-672. https://doi.org/10.1037/00223514.69.4.656

Gosling, S. D., Ko, S. J., Mannarelli, T., \& Morris, M. E. (2002). A room with a cue: Personality judgments based on offices and bedrooms. Journal of Personality and Social Psychology, 82, 379-398. https://doi.org/10.1037//0022-3514.82.3.379

Graham, L. T., \& Gosling, S. D. (2012). Impressions of World of Warcraft players' personalities based on their usernames: Interobserver consensus but no accuracy. Journal of Research in Personality, 46, 599-603. https://doi.org/10.1016/j.jrp.2012.05.002

Grice, H. P. (1975). Logic and Conversation. In P. Cole \& J. L. Morgan (Eds.), Syntax and Semantics 3: Speech acts (pp. 41-58). New York: Academic Press. https://doi.org/10.1111/j.1365-2664.2006.01229.x

Harari, G. M., Graham, L. T., \& Gosling, S. D. (2015). Personality impressions of world of Warcraft players based on their avatars and usernames: Consensus but no accuracy. International Journal of Gaming and Computer-Mediated Simulations, 7, 58-73. https://doi.org/10.4018/IJGCMS.2015010104

Hogan, R. (1996). A Socioanalytic perspective on five factor model. In J. S. Wiggins (Ed.), The five-factor model of personality: Theoretical Perspectives (pp. 163-179). New York: Guilford Press. 


\section{NETWORK APPROACH TO REPUTATIONS}

Human, L. J., \& Biesanz, J. C. (2011). Target adjustment and self-other agreement: Utilizing trait observability to disentangle judgeability and self-knowledge. Journal of Personality and Social Psychology, 101, 202-216. https://doi.org/10.1037/a0023782

Human, L. J., \& Biesanz, J. C. (2012). Accuracy and assumed similarity in first impressions of personality: Differing associations at different levels of analysis. Journal of Research in Personality, 46(1), 106-110. https://doi.org/10.1016/j.jrp.2011.10.002

Human, L. J., \& Biesanz, J. C. (2013). Targeting the Good Target. Personality and Social Psychology Review, 17, 248-272. https://doi.org/10.1177/1088868313495593

John, O. P., \& Robins, R. W. (1993). Determinants of Interjudge Agreement on Personality Traits: The Big Five Domains, Observability, Evaluativeness, and the Unique Perspective of the Self. Journal of Personality, 61, 521-551. https://doi.org/10.1111/j.14676494.1993.tb00781.x

John, O. P., \& Robins, R. W. (1994). Accuracy and bias in self-perception: individual differences in self-enhancement and the role of narcissism. Journal of Personality and Social Psychology, 66, 206-219. https://doi.org/10.1037/0022-3514.66.1.206

Judge, T. A., Bono, J. E., Ilies, R., \& Gerhardt, M. W. (2002). Personality and leadership: A qualitative and quantitative review. Journal of Applied Psychology, 87, 542-552. https://doi.org/10.1037//0021-9010.87.4.765

Kadushin, C. (2012). Understanding social networks: Theories, concepts, and findings. New York, NY: Oxford University Press.

Kenny, D. A. (1994). Interpersonal Perception: A social relations analysis. New York, NY: The Guilford Press.

Kenny, D. A. (2004). PERSON: a general model of interpersonal perception. Personality and Social Psychology Review, 8, 265-280. https://doi.org/10.1207/s15327957pspr0803_3

Kuznetsova A., Brockhoff, P. B., Christensen, R. H. B. (2017). lmerTest Package: Tests in Linear Mixed Effects Models. Journal of Statistical Software, 82, 1-26. https://doi.org/10.18637/jss.v082.i13

Kwan, V. S. Y., John, O. P., Kenny, D. a, Bond, M. H., \& Robins, R. W. (2004). Reconceptualizing individual differences in self-enhancement bias: an interpersonal approach. Psychological Review, 111, 94-110. https://doi.org/10.1037/0033295X.111.1.94

Leising, D., Erbs, J., \& Fritz, U. (2010). The letter of recommendation effect in informant ratings of personality. Journal of Personality and Social Psychology, 98, 668-682. https://doi.org/10.1037/a0018771 
NETWORK APPROACH TO REPUTATIONS

Leising, D., Locke, K. D., Kurzius, E., \& Zimmermann, J. (2016). Quantifying the Association of Self-Enhancement Bias with Self-Ratings of Personality and Life Satisfaction. Assessment, 23, 588-602. https://doi.org/10.1177/1073191115590852

MacCoun, R., \& Perlmutter, S. (2015). Hide results to seek the truth. Nature, 526, 187-189. https://doi.org/10.1038/526187a

Malloy, T. E., Agatstein, F., Yarlas, a, \& Albright, L. (1997). Effects of communication, information overlap, and behavioral consistency on consensus in social perception. Journal of Personality and Social Psychology, 73, 270-280. https://doi.org/10.1037//0022-3514.73.2.270

McAbee, S. T., \& Connelly, B. S. (2016). A Multi-Rater Framework for Studying Personality: The Trait-Reputation-Identity Model. Psychological Review, 123. https://doi.org/10.1037/rev0000035

Murray, S. L., Holmes, J. G., \& Griffin, D. W. (1996). The benefits of positive illusions: Idealization and the construction of satisfaction in close relationships. Journal of Personality and Social Psychology, 70(1), 79-98. https://doi.org/10.1037/0022$\underline{3514.70 .1 .79}$

Muthén, L. K. \& Muthén, B. O. (1998-2012). Mplus user's guide. Seventh Edition. Los Angeles, CA: Muthén \& Muthén.

Olsen, J. A., \& Kenny, D. A. (2006). Structural equation modeling with interchangeable dyads. Psychological Methods, 11, 127-141. https://doi.org/10.1037/1082-989X.11.2.127

Paulhus, D. L., \& John, O. P. (1998). Egoistic and moralistic biases in self-perception: The interplay of self-deceptive styles with basic traits and motives. Journal of Personality, 666, 1025-1060. https://doi.org/10.1111/1467-6494.00041

R Core Team (2019). R: A language and environment for statistical computing. R Foundation for Statistical Computing, Vienna, Austria. https:/www.R-project.org/.

Robins, R., \& John, O. (1997). The quest for self-insight: Theory and research on accuracy and bias in self-perception. In R. Hogan, J. Johnson, S. Briggs (Eds.), Handbook of Personality Psychology, 649-679. https://doi.org/10.1016/B978-012134645-4/50026-3

Rosseel, Y. (2012). lavaan: An R Package for Structural Equation Modeling. Journal of Statistical Software, 48(2), 1-36. URL http://www.jstatsoft.org/v48/i02/.

Saucier, G., \& Goldberg, L. R. (1996). The Language of Personality: Lexical Perspectives on the Five-Factor Model. In The five-factor model of personality: Theoretical perspectives (pp. 21-50). New York: Guilford Press. 


\section{NETWORK APPROACH TO REPUTATIONS}

Saucier, G. \& Srivastava, S. (2015). What makes a good structural model of personality? Evaluating the big five and alternatives. In M. Mikulincer, P. R. Shaver, M. L. Cooper \& R. J. Larsen (Eds.), Handbook of personality and social psychology. Vol. 3: Personality processes and individual differences (pp. 283-305). Washington, DC: APA Books.

Soto, C. J., \& John, O. P. (2017). The next big five inventory (BFI-2): Developing and assessing a hierarchical model with 15 facets to enhance bandwidth, fidelity, and predictive power. Journal of Personality and Social Psychology. 117-143. https://doi.org/http://dx.doi.org/10.1037/pspp0000096

Srivastava, S. (2010). The Five-Factor Model Describes the Structure of Social Perceptions. Psychological Inquiry, 21, 69-75. https://doi.org/10.1080/10478401003648815

Thalmayer, A. G., Saucier, G., \& Eigenhuis, A. (2011). Comparative validity of Brief to Medium-Length Big Five and Big Six Personality Questionnaires. Psychological Assessment, 23(4), 995-1009. https://doi.org/10.1037/a0024165

Vazire, S. (2010). Who knows what about a person? The self-other knowledge asymmetry (SOKA) model. Journal of Personality and Social Psychology, 98, 281-300. https://doi.org/10.1037/a0017908

Wessels, N. M., Zimmermann, J., Biesanz, J. C., \& Leising, D. (2020). Differential Associations of Knowing and Liking with Accuracy and Positivity Bias in Person Perception. Journal of Personality and Social Psychology, 118(1), 149-171. https://doi.org/10.1037/pspp0000218

Wood, D. (2015). Testing the lexical hypothesis: Are socially important traits more densely reflected in the English lexicon? Journal of Personality and Social Psychology, 108(2), 317-335. https://doi.org/10.1037/a0038343

Wood, D., Tov, W., Costello, C., Tov, W., \& Costello, C. (2015). What a__ Thing to Do ! Formally Characterizing Actions by Their Expected Effects. Journal of Personality and Social Psychology, 108, 953-976. http://dx.doi.org/10.1037/pspp0000030

Wortman, J., \& Wood, D. (2011). The personality traits of liked people. Journal of Research in Personality, 45, 519-528. https://doi.org/10.1016/j.jrp.2011.06.006

Wu, J., Balliet, D., \& Van Lange, P. A. M. (2015). When Does Gossip Promote Generosity? Indirect Reciprocity Under the Shadow of the Future. Social Psychological and Personality Science, 6(8), 923-930. https://doi.org/10.1177/1948550615595272

Zimmermann, J., Schindler, S., Klaus, G., \& Leising, D. (2018). The Effect of Dislike on Accuracy and Bias in Person Perception. Social Psychological and Personality Science, 9(1), 80-88. https://doi.org/10.1177/1948550617703167 
NETWORK APPROACH TO REPUTATIONS: SUPPLEMENT

\section{Assessing Justifiability of Integrating Across Studies}

We assessed the justifiability of integrating across studies for both the SAM and domainwise correlation analyses using model comparisons. We combined the data from both studies and estimated two models for each analysis: a non-integrated model, which allowed all model parameters to differ across studies, and an integrated model, which constrained all model parameters to be equal across studies. These were omnibus tests; they tested whether or not all parameters in the model could be pooled.

For SAM, this consisted of comparing models with and without random effects (on all parameters) for study, separately for the consensus and accuracy models. The model without random effects for study fit the data better by every metric we examined for the consensus model $\left(\chi^{2}{ }_{\mathrm{d}}(10)=6.04, p=.812, \Delta_{\mathrm{AIC}}=-13.96, \Delta_{\mathrm{BIC}}=-92.14\right)$ and the accuracy model $\left(\chi_{\mathrm{d}}^{2}(36)=4.35\right.$, $\left.p=1, \Delta_{\mathrm{AIC}}=-67.65, \Delta_{\mathrm{BIC}}=-378.90\right)$, lending unequivocal support for integrating across studies for all of the SAM analyses.

For domain-wise correlations, we used multi-group path analytic framework to test whether the full variance-covariance and mean structure of the ratings could be pooled across studies, effectively testing whether all analyses could be integrated across studies in one test (for each Big Six domain). The results of these comparisons are shown in Supplemental Table S38 below, where it is apparent that all model comparison results lend unequivocal support to integrating across studies for domain-wise correlations. 
NETWORK APPROACH TO REPUTATIONS: SUPPLEMENT

Table S1

Coefficient Alphas for Big Six Ratings

\begin{tabular}{llcc}
\hline Report Type & Domain & Study $1 \alpha$ & Study $2 \alpha$ \\
\hline Self & agreeableness & .76 & .76 \\
& conscientiousness & .83 & .86 \\
& honesty-propriety & .61 & .62 \\
neuroticism & .89 & .88 \\
extraversion & .87 & .87 \\
& openness & .81 & .81 \\
agreeableness & .80 & .82 \\
P1 & conscientiousness & .83 & .88 \\
& honesty-propriety & .66 & .76 \\
& neuroticism & .75 & .84 \\
& extraversion & .88 & .88 \\
& openness & .78 & .87 \\
P2 & agreeableness & .84 & .83 \\
& conscientiousness & .89 & .91 \\
& honesty-propriety & .75 & .79 \\
& neuroticism & .87 & .83 \\
& extraversion & .89 & .91 \\
& openness & .83 & .77 \\
\hline
\end{tabular}

Note. For self-, P1-, and P2-reports, each of the Big Five was measured with 12 items and honesty-propriety with 8 items. 
NETWORK APPROACH TO REPUTATIONS: SUPPLEMENT

Table S2

Uncentered Mean Ratings for Big Six Items by Rater Type

\begin{tabular}{|c|c|c|c|c|c|}
\hline Item & Text & Self & P1 & P2 & Desirability \\
\hline bfi_1 & Is Outgoing, Sociable. & 3.93 & 3.86 & 3.71 & 4.46 \\
\hline bfi_2 2 & Is Compassionate, has a soft heart. & 4.43 & 4.23 & 4.00 & 4.54 \\
\hline bfi_3 & Tends to be disorganized. & 2.86 & 2.41 & 2.29 & 2.00 \\
\hline bfi_ 4 & Is relaxed, handles stress well. & 3.07 & 3.57 & 3.39 & 4.54 \\
\hline bfi 5 & Has few artistic interests. & 2.72 & 2.84 & 2.96 & 2.46 \\
\hline bfi_6 & Has an assertive personality. & 3.09 & 2.79 & 2.88 & 3.15 \\
\hline bfi_7 & Is respectful, treats others with respect. & 4.71 & 4.53 & 4.31 & 4.85 \\
\hline bfi_8 & Tends to be lazy. & 3.15 & 2.35 & 2.12 & 1.46 \\
\hline bfi_9 & Stays optimistic after experiencing a setback. & 3.61 & 3.73 & 3.55 & 4.62 \\
\hline bfi_-10 & Is curious about many different things. & 4.41 & 3.88 & 3.71 & 4.31 \\
\hline bfi_11 & Rarely feels excited or eager. & 2.09 & 2.35 & 2.43 & 1.92 \\
\hline bfi_12 & Tends to find fault with others. & 2.65 & 2.32 & 2.44 & 1.31 \\
\hline bfi_13 & Is dependable, steady. & 4.20 & 3.95 & 3.89 & 4.62 \\
\hline bfi_14 & Is moody, has up and down mood swings. & 2.89 & 2.42 & 2.54 & 1.92 \\
\hline bfi_15 & Is inventive, finds clever ways to do things. & 3.70 & 3.39 & 3.29 & 4.38 \\
\hline bfi_- 16 & Tends to be quiet. & 3.17 & 3.14 & 3.10 & 2.62 \\
\hline bfi_17 & Feels little sympathy for others. & 2.68 & 2.73 & 2.90 & 1.38 \\
\hline bfi_-18 & Is systematic, likes to keep things in order. & 3.78 & 3.80 & 3.71 & 3.92 \\
\hline bfi_19 & Can be tense. & 3.65 & 3.04 & 3.11 & 2.23 \\
\hline bfi_-20 & Is fascinated by art, music, or literature. & 3.90 & 3.44 & 3.28 & 3.69 \\
\hline bfi_21 & Is dominant, acts as a leader. & 3.36 & 2.83 & 2.94 & 3.38 \\
\hline bfi_22 & Starts arguments with others. & 2.09 & 2.11 & 2.20 & 1.46 \\
\hline bfi_23 & Has difficulty getting started on tasks. & 3.32 & 2.59 & 2.49 & 2.08 \\
\hline bfi_-24 & Feels secure, comfortable with self. & 3.65 & 3.83 & 3.63 & 4.92 \\
\hline bfi_-25 & Avoids intellectual, philosophical discussions. & 2.09 & 2.41 & 2.57 & 2.08 \\
\hline bfi_-26 & Is less active than other people. & 2.44 & 2.38 & 2.42 & 2.31 \\
\hline bfi_-27 & Has a forgiving nature. & 4.19 & 4.08 & 3.77 & 4.38 \\
\hline bfi_-28 & Can be somewhat careless. & 3.00 & 2.66 & 2.61 & 2.23 \\
\hline bfi_-29 & Is emotionally stable, not easily upset. & 3.31 & 3.64 & 3.40 & 4.46 \\
\hline bfi_30 & Has little creativity. & 2.33 & 2.42 & 2.62 & 2.31 \\
\hline bfi_31 & Is sometimes shy, introverted. & 3.70 & 3.52 & 3.34 & 2.69 \\
\hline bfi_32 & Is helpful and unselfish with others. & 4.23 & 4.01 & 3.69 & 4.69 \\
\hline bfi_33 & Keeps things neat and tidy. & 3.48 & 3.75 & 3.61 & 3.77 \\
\hline bfi_34 & Worries a lot. & 3.79 & 3.22 & 3.10 & 2.15 \\
\hline bfi_35 & Values art and beauty. & 4.08 & 3.49 & 3.36 & 3.85 \\
\hline bfi_-36 & Finds it hard to influence people. & 2.58 & 3.03 & 2.90 & 2.77 \\
\hline bfi_37 & Is sometimes rude to others. & 2.55 & 2.37 & 2.41 & 1.77 \\
\hline bfi_38 & Is efficient, gets things done. & 4.00 & 3.92 & 3.86 & 4.23 \\
\hline bfi_39 & Often feels sad. & 2.90 & 2.59 & 2.70 & 2.38 \\
\hline bfi_40 & Is complex, a deep thinker. & 4.08 & 3.49 & 3.47 & 4.15 \\
\hline bfi_ 41 & Is full of energy. & 3.57 & 3.44 & 3.54 & 4.31 \\
\hline bfi_ 42 & Is suspicious of others' intentions. & 3.45 & 2.70 & 2.86 & 2.00 \\
\hline
\end{tabular}


bfi_43 Is reliable, can always be counted on.

$\begin{array}{llll}4.31 & 3.84 & 3.74 & 4.85\end{array}$

bfi_44 Keeps their emotions under control.

$\begin{array}{llll}3.67 & 3.87 & 3.51 & 4.00\end{array}$

bfi_45 Has difficulty imagining things.

$\begin{array}{llll}2.02 & 2.57 & 2.63 & 2.54\end{array}$

bfi_46 Is talkative.

$\begin{array}{llll}3.86 & 3.63 & 3.49 & 3.08\end{array}$

bfi_47 Can be cold and uncaring.

$\begin{array}{llll}2.23 & 1.98 & 2.21 & 1.38\end{array}$

bfi_48 Leaves a mess, doesn't clean up.

$\begin{array}{llll}1.87 & 2.11 & 2.28 & 1.69\end{array}$

bfi 49 Rarely feels anxious or afraid.

$\begin{array}{llll}2.50 & 2.78 & 2.79 & 3.62\end{array}$

bfi_50 Thinks poetry and plays are boring.

$\begin{array}{llll}2.69 & 2.82 & 2.89 & 2.31\end{array}$

bfi_51 Prefers to have others take charge.

$\begin{array}{lll}2.83 & 3.13 & 2.99\end{array}$

2.46

bfi_52 Is polite, courteous to others.

$\begin{array}{llll}4.60 & 4.44 & 4.16 & 4.62\end{array}$

bfi_53 Is persistent, works until task is finished.

$\begin{array}{lll}3.98 & 3.99 & 3.85\end{array}$

4.54

bfi_54 Tends to feel depressed, blue.

$\begin{array}{llll}2.62 & 2.45 & 2.54 & 2.15\end{array}$

bfi_55 Has little interest in abstract ideas.

$\begin{array}{llll}2.31 & 2.60 & 2.77 & 2.46\end{array}$

bfi_56 Shows a lot of enthusiasm.

$\begin{array}{llll}3.78 & 3.51 & 3.55 & 4.31\end{array}$

bfi_57 Assumes the best about people.

$\begin{array}{lll}3.84 & 3.82 & 3.62\end{array}$

4.46

bfi_58 Sometimes behaves irresponsibly.

$\begin{array}{llll}3.25 & 2.77 & 2.82 & 2.00\end{array}$

bfi_59 Is temperamental, gets emotional easily. $\quad \begin{array}{lllll}2.77 & 2.56 & 2.75 & 1.62\end{array}$

bfi_60 Is original, comes up with new ideas. $\quad \begin{array}{lllll}- & 3.59 & 3.35 & 3.25 & 4.15\end{array}$

$\begin{array}{llllll}\text { hp_1 } & \text { Uses flattery to get ahead. } & 2.62 & 2.24 & 2.50 & 1.85\end{array}$

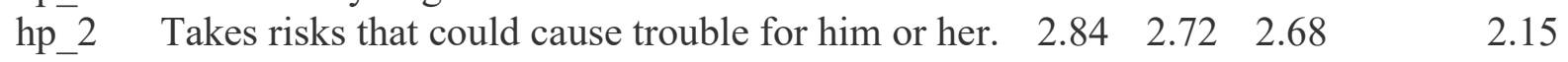

hp_3 Uses others for his or her own ends. $\quad 2.02 \quad 2.05 \quad 2.21 \quad 1.15$

$\begin{array}{llllll}\text { hp_4 } & \text { Misrepresents the facts. } & 2.16 & 2.22 & 2.48 & 1.15\end{array}$

hp_5 Has bad manners. $\quad \begin{array}{lllll}1.51 & 1.47 & 2.04 & 1.25\end{array}$

hp_6 $\quad$ Would never take things that aren't their own. $\quad \begin{array}{lllll}4.12 & 3.82 & 3.57 & 4.23\end{array}$

$\begin{array}{llllll}\text { hp_7 } & \text { Sticks to the rules. } & 3.58 & 3.62 & 3.34 & 3.38\end{array}$

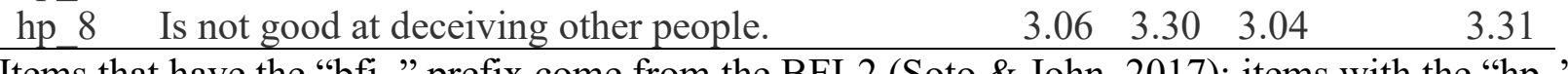
Items that have the "bfi_" prefix come from the BFI-2 (Soto \& John, 2017); items with the "hp_" prefix are honesty-propriety items from the QB6 family of measures (Thalmayer, Saucier, \& Eigenhuis, 2011). All ratings were completed on 1 to 5 likert scales. 
NETWORK APPROACH TO REPUTATIONS: SUPPLEMENT

Table S3

Example Variance-Covariance Matrix

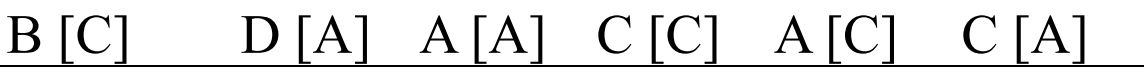

$\begin{array}{lll}\text { B }[\text { C }] & \text { P2 } & \\ \text { D [A] } & \text { h } & \text { P2 }\end{array}$

$\begin{array}{lllll}\text { A [A] } & \text { j } & \text { e } & \text { self } & \\ \text { C [C] } & \text { e } & j & \text { i } & \text { self }\end{array}$

$\begin{array}{lllllll}\mathrm{A}[\mathrm{C}] & \mathrm{f} & \mathrm{m} & \mathrm{l} & \mathrm{g} & \mathrm{P} 1 & \\ \mathrm{C}[\mathrm{A}] & \mathrm{m} & \mathrm{f} & \mathrm{g} & \mathrm{l} & \mathrm{k} & \mathrm{P} 1\end{array}$

Note. Letters in the row and column labels correspond to the names in the graphical depiction of the study design in Figure 1a. The perceiver's procedural role appears outside of the brackets and the target's procedural role appears inside of the brackets, e.g., A [C] is A's perception of C. Cells with the same letter are conceptually identical and pooled in our analyses (this includes variances with the same label). Intercept equality constraints are not pictured, but ratings which have equality constraints on variances would also have equality constraints on intercepts. Cells with gray backgrounds are unique (i.e., they do not have a conceptually identical estimate elsewhere in the model). 
NETWORK APPROACH TO REPUTATIONS: SUPPLEMENT

Table S4

Random Effects for Hearsay Consensus SAM

\begin{tabular}{lllr}
\hline source & type & term & Est. \\
\hline triad:group & variance & Intercept & 0.00 \\
triad:group & variance & Normative Consensus & 0.15 \\
triad:group & variance & Distinctive Consensus & 0.02 \\
triad:group & variance & Positivity Bias & 0.10 \\
triad:group & covariance & Intercept, Normative Consensus & -0.02 \\
triad:group & covariance & Intercept, Distinctive Consensus & 0.00 \\
triad:group & covariance & Intercept, Positivity Bias & 0.00 \\
triad:group & covariance & Normative Consensus, Distinctive Consensus & 0.00 \\
triad:group & covariance & Normative Consensus, Positivity Bias & -0.06 \\
triad:group & covariance & Distinctive Consensus, Positivity Bias & 0.00 \\
group & variance & Intercept & 0.00 \\
group & variance & Normative Consensus & 0.05 \\
group & variance & Distinctive Consensus & 0.00 \\
group & variance & Positivity Bias & 0.01 \\
group & covariance & Intercept, Normative Consensus & 0.00 \\
group & covariance & Intercept, Distinctive Consensus & 0.00 \\
group & covariance & Intercept, Positivity Bias & 0.00 \\
group & covariance & Normative Consensus, Distinctive Consensus & 0.01 \\
group & covariance & Normative Consensus, Positivity Bias & -0.02 \\
group & covariance & Distinctive Consensus, Positivity Bias & 0.00 \\
Residual & & & 0.69 \\
\hline & & & \\
\hline
\end{tabular}


Table S5

Random Effects for Hearsay and Direct Accuracy SAM

\begin{tabular}{|c|c|c|c|}
\hline source & type & term & Est. \\
\hline triad:group & variance & Intercept & 0.01 \\
\hline triad:group & variance & Normative Accuracy & 0.06 \\
\hline triad:group & variance & Distinctive Accuracy & 0.01 \\
\hline triad:group & variance & distance (p1 vs. p2) & 0.01 \\
\hline triad:group & variance & Normative Accuracy X distance (p1 vs. p2) & 0.06 \\
\hline triad:group & variance & Distinctive Accuracy X distance (p1 vs. $\mathrm{p} 2)$ & 0.01 \\
\hline triad:group & variance & distancep1 X Positivity Bias & 0.08 \\
\hline triad:group & variance & distance (p1 vs. p2) X Positivity Bias & 0.09 \\
\hline triad:group & covariance & Intercept, Normative Accuracy & 0.01 \\
\hline triad:group & covariance & Intercept, Distinctive Accuracy & 0.00 \\
\hline triad:group & covariance & Intercept, distance (p1 vs. p2) & -0.01 \\
\hline triad:group & covariance & Intercept, Normative Accuracy X distance (p1 vs. p2) & -0.01 \\
\hline triad:group & covariance & Intercept, Distinctive Accuracy X distance (p1 vs. p2) & 0.00 \\
\hline triad:group & covariance & Intercept, distancep $1 \mathrm{X}$ Positivity Bias & -0.01 \\
\hline triad:group & covariance & Intercept, distance (p1 vs. p2) X Positivity Bias & 0.00 \\
\hline triad:group & covariance & Normative Accuracy, Distinctive Accuracy & 0.01 \\
\hline triad:group & covariance & Normative Accuracy, distance (p1 vs. p2) & -0.01 \\
\hline & & Normative Accuracy, Normative Accuracy X distance (p1 & \\
\hline triad:group & covariance & $\begin{array}{l}\text { vs. p2) } \\
\text { Normative Accuracy, Distinctive Accuracy X distance (p1 }\end{array}$ & -0.05 \\
\hline triad:group & covariance & vs. p2) & 0.00 \\
\hline triad:group & covariance & $\begin{array}{l}\text { Normative Accuracy, distancep1 X Positivity Bias } \\
\text { Normative Accuracy, distance (p1 vs. p2) X Positivity }\end{array}$ & -0.02 \\
\hline triad:group & covariance & Bias & -0.01 \\
\hline triad:group & covariance & $\begin{array}{l}\text { Distinctive Accuracy, distance ( } \mathrm{p} 1 \text { vs. } \mathrm{p} 2) \\
\text { Distinctive Accuracy, Normative Accuracy X distance (p1 }\end{array}$ & 0.00 \\
\hline triad:group & covariance & $\begin{array}{l}\text { vs. p2) } \\
\text { Distinctive Accuracy, Distinctive Accuracy X distance (p1 }\end{array}$ & 0.00 \\
\hline triad:group & covariance & vs. p2) & 0.00 \\
\hline triad:group & covariance & $\begin{array}{l}\text { Distinctive Accuracy, distancep1 X Positivity Bias } \\
\text { Distinctive Accuracy, distance (p1 vs. p2) X Positivity }\end{array}$ & 0.00 \\
\hline triad:group & covariance & $\begin{array}{l}\text { Bias } \\
\text { distance (p1 vs. p2), Normative Accuracy X distance (p1 }\end{array}$ & 0.00 \\
\hline triad:group & covariance & $\begin{array}{l}\text { vs. p2) } \\
\text { distance (p1 vs. p2), Distinctive Accuracy X distance ( } 1 \text { 1 }\end{array}$ & 0.01 \\
\hline triad:group & covariance & Vs. p2) & 0.00 \\
\hline triad:group & covariance & distance ( $\mathrm{p} 1$ vs. $\mathrm{p} 2$ ), distancep1 X Positivity Bias & 0.01 \\
\hline triad:group & covariance & $\begin{array}{l}\text { distance (p1 vs. } \mathrm{p} 2 \text { ), distance ( } 1 \text { vs. p2) X Positivity Bias } \\
\text { Normative Accuracy X distance (p1 vs. p2), Distinctive }\end{array}$ & -0.01 \\
\hline triad:group & covariance & Accuracy X distance (p1 vs. p2) & 0.01 \\
\hline
\end{tabular}




\begin{tabular}{|c|c|c|c|}
\hline triad:group & covariance & $\begin{array}{l}\text { Normative Accuracy X distance (p1 vs. p2), distancep1 X } \\
\text { Positivity Bias }\end{array}$ & 0.01 \\
\hline \multirow{3}{*}{ triad:group } & \multirow{3}{*}{ covariance } & Normative Accuracy X distance ( $\mathrm{p} 1$ vs. $\mathrm{p} 2)$, distance ( $\mathrm{p} 1$ & \multirow{3}{*}{-0.01} \\
\hline & & vs. p2) X Positivity Bias & \\
\hline & & Distinctive Accuracy X distance (p1 vs. $\mathrm{p} 2$ ), distancep1 X & \\
\hline \multirow[t]{2}{*}{ triad:group } & \multirow[t]{2}{*}{ covariance } & Positivity Bias & \multirow[t]{2}{*}{0.01} \\
\hline & & Distinctive Accuracy X distance (p1 vs. $\mathrm{p} 2)$, distance ( $\mathrm{p} 1$ & \\
\hline triad:group & covariance & $\begin{array}{l}\text { vs. p2) X Positivity Bias } \\
\text { distancep1 X Positivity Bias, distance (p1 vs. p2) X }\end{array}$ & 0.00 \\
\hline triad:group & covariance & Positivity Bias & 0.03 \\
\hline group & variance & Intercept & 0.00 \\
\hline group & variance & Normative Accuracy & 0.01 \\
\hline group & variance & Distinctive Accuracy & 0.00 \\
\hline group & variance & distance (p1 vs. p2) & 0.00 \\
\hline group & variance & Normative Accuracy X distance (p1 vs. p2) & 0.00 \\
\hline group & variance & Distinctive Accuracy X distance (p1 vs. p2) & 0.00 \\
\hline group & variance & distancep1 X Positivity Bias & 0.00 \\
\hline group & variance & distance (p1 vs. p2) X Positivity Bias & 0.00 \\
\hline group & covariance & Intercept, Normative Accuracy & 0.00 \\
\hline group & covariance & Intercept, Distinctive Accuracy & 0.00 \\
\hline group & covariance & Intercept, distance (p1 vs. p2) & 0.00 \\
\hline group & covariance & Intercept, Normative Accuracy X distance (p1 vs. p2) & 0.00 \\
\hline group & covariance & Intercept, Distinctive Accuracy X distance (p1 vs. p2) & 0.00 \\
\hline group & covariance & Intercept, distancep $1 \mathrm{X}$ Positivity Bias & 0.00 \\
\hline group & covariance & Intercept, distance (p1 vs. p2) X Positivity Bias & 0.00 \\
\hline group & covariance & Normative Accuracy, Distinctive Accuracy & 0.00 \\
\hline group & covariance & Normative Accuracy, distance (p1 vs. p2) & 0.00 \\
\hline & & Normative Accuracy, Normative Accuracy X distance (p1 & \\
\hline group & covariance & vs. p2) & 0.00 \\
\hline & & Normative Accuracy, Distinctive Accuracy X distance (p1 & \\
\hline group & covariance & vs. p2) & 0.00 \\
\hline group & covariance & Normative Accuracy, distancep1 X Positivity Bias & 0.00 \\
\hline & & Normative Accuracy, distance (p1 vs. p2) X Positivity & \\
\hline group & covariance & Bias & 0.00 \\
\hline group & covariance & Distinctive Accuracy, distance (p1 vs. p2) & 0.00 \\
\hline & & Distinctive Accuracy, Normative Accuracy X distance (p1 & \\
\hline group & covariance & vs. p2) & 0.00 \\
\hline & & Distinctive Accuracy, Distinctive Accuracy X distance (p1 & \\
\hline group & covariance & vs. p2) & 0.00 \\
\hline group & covariance & Distinctive Accuracy, distancep1 X Positivity Bias & 0.00 \\
\hline & & Distinctive Accuracy, distance (p1 vs. p2) X Positivity & \\
\hline group & covariance & $\begin{array}{l}\text { Bias } \\
\text { distance (p1 vs. p2), Normative Accuracy X distance (p1 }\end{array}$ & 0.00 \\
\hline group & covariance & vs. p2) & 0.00 \\
\hline
\end{tabular}




\begin{tabular}{|c|c|c|c|}
\hline group & covariance & $\begin{array}{l}\text { distance (p1 vs. p2), Distinctive Accuracy X distance (p1 } \\
\text { vs. p2) }\end{array}$ & 0.00 \\
\hline group & covariance & distance ( $\mathrm{p} 1$ vs. $\mathrm{p} 2$ ), distancep1 X Positivity Bias & 0.00 \\
\hline group & covariance & $\begin{array}{l}\text { distance ( } 1 \text { vs. } \mathrm{p} 2 \text { ), distance ( } 1 \text { 1 vs. } \mathrm{p} 2) \text { X Positivity Bias } \\
\text { Normative Accuracy X distance (p1 vs. p2), Distinctive }\end{array}$ & 0.00 \\
\hline group & covariance & $\begin{array}{l}\text { Accuracy X distance ( } \mathrm{p} 1 \text { vs. } \mathrm{p} 2) \\
\text { Normative Accuracy X distance (p1 vs. } \mathrm{p} 2) \text {, distancep1 X }\end{array}$ & 0.00 \\
\hline group & covariance & $\begin{array}{l}\text { Positivity Bias } \\
\text { Normative Accuracy X distance (p1 vs. p2), distance (p1 }\end{array}$ & 0.00 \\
\hline group & covariance & $\begin{array}{l}\text { vs. p2) X Positivity Bias } \\
\text { Distinctive Accuracy X distance (p1 vs. p2), distancep1 X }\end{array}$ & 0.00 \\
\hline group & covariance & $\begin{array}{l}\text { Positivity Bias } \\
\text { Distinctive Accuracy X distance (p1 vs. p2), distance (p1 }\end{array}$ & 0.00 \\
\hline group & covariance & $\begin{array}{l}\text { vs. p2) X Positivity Bias } \\
\text { distancep1 X Positivity Bias, distance (p1 vs. p2) X }\end{array}$ & 0.00 \\
\hline $\begin{array}{l}\text { group } \\
\text { Residual }\end{array}$ & covariance & Positivity Bias & $\begin{array}{l}0.00 \\
0.78\end{array}$ \\
\hline
\end{tabular}


NETWORK APPROACH TO REPUTATIONS: SUPPLEMENT

Table S6

Random Effects for Simple Hearsay Accuracy SAM

\begin{tabular}{lllr}
\hline source & type & term & Est. \\
\hline triad:group & variance & Intercept & 0.00 \\
triad:group & variance & Normative Accuracy & 0.04 \\
triad:group & variance & Distinctive Accuracy & 0.01 \\
triad:group & variance & Positivity Bias & 0.09 \\
triad:group & covariance & Intercept, Normative Accuracy & 0.00 \\
triad:group & covariance & Intercept, Distinctive Accuracy & 0.00 \\
triad:group & covariance & Intercept, Positivity Bias & -0.01 \\
triad:group & covariance & Normative Accuracy, Distinctive Accuracy & 0.01 \\
triad:group & covariance & Normative Accuracy, Positivity Bias & -0.02 \\
triad:group & covariance & Distinctive Accuracy, Positivity Bias & 0.00 \\
group & variance & Intercept & 0.00 \\
group & variance & Normative Accuracy & 0.01 \\
group & variance & Distinctive Accuracy & 0.00 \\
group & variance & Positivity Bias & 0.00 \\
group & covariance & Intercept, Normative Accuracy & 0.00 \\
group & covariance & Intercept, Distinctive Accuracy & 0.00 \\
group & covariance & Intercept, Positivity Bias & 0.00 \\
group & covariance & Normative Accuracy, Distinctive Accuracy & 0.00 \\
group & covariance & Normative Accuracy, Positivity Bias & 0.00 \\
group & covariance & Distinctive Accuracy, Positivity Bias & 0.00 \\
Residual & & &
\end{tabular}


NETWORK APPROACH TO REPUTATIONS: SUPPLEMENT

Table S7

Fixed Effects for Target Extraversion Moderating Hearsay Consensus (SAM)

\begin{tabular}{|c|c|c|c|c|c|c|c|c|c|}
\hline term & component & Est. & CI LL & CI UL & SE & $t$ & $d f$ & $p$ & $\begin{array}{l}\text { Std. } \\
\text { Est }\end{array}$ \\
\hline$\gamma_{000}$ & intercept & 0.00 & -0.01 & 0.02 & 0.01 & 0.30 & 149.02 & .763 & .00 \\
\hline$\gamma_{100}$ & Normative Consensus & 0.72 & 0.65 & 0.79 & 0.03 & 20.68 & 302.23 & $<.001$ & .46 \\
\hline$\gamma_{200}$ & Distinctive Consensus & 0.21 & 0.18 & 0.23 & 0.01 & 16.59 & 175.45 & $<.001$ & .18 \\
\hline$\gamma_{300}$ & Positivity Bias & 0.06 & 0.02 & 0.10 & 0.02 & 3.16 & 536.93 & .002 & .07 \\
\hline$\gamma_{400}$ & Target Extraversion & 0.03 & .000 & 0.05 & 0.01 & 2.12 & 73.88 & .037 & .02 \\
\hline \multirow[t]{2}{*}{$\gamma_{110}$} & Target Extraversion $\mathrm{X}$ & & & & & & & & \\
\hline & Normative Consensus & -0.11 & -0.21 & -0.01 & 0.05 & -2.13 & 99.76 & .035 & -.05 \\
\hline \multirow[t]{2}{*}{$\gamma_{210}$} & Target Extraversion $\mathrm{X}$ & & & & & & & & \\
\hline & Distinctive Consensus & 0.01 & -0.03 & 0.04 & 0.02 & 0.36 & 175.10 & .716 & .00 \\
\hline \multirow[t]{2}{*}{$\gamma_{310}$} & Target Extraversion $\mathrm{X}$ & & & & & & & & \\
\hline & Positivity Bias & 0.07 & -0.01 & 0.16 & 0.04 & 1.78 & 121.52 & .078 & .06 \\
\hline
\end{tabular}

Note. Term refers to the term in the corresponding equation. Component refers to the SAM component. Est. refers to the unstandardized estimate. CI LL and CI UL refer to the lower and upper limits of the $95 \%$ CI respectively. SE refers to standard error. $d f$ were calculated using the Satterthwaite approximation. Std. Est. refers to the standardized estimates.

Table S8

Fixed Effects for P1 Extraversion Moderating Hearsay Consensus (SAM)

\begin{tabular}{|c|c|c|c|c|c|c|c|c|c|}
\hline term & component & Est. & CI LL & CI UL & SE & $t$ & $d f$ & $p$ & $\begin{array}{l}\text { Std. } \\
\text { Est }\end{array}$ \\
\hline$\gamma_{000}$ & intercept & 0.01 & -0.01 & 0.02 & 0.01 & 0.67 & 166.14 & 0.506 & .00 \\
\hline$\gamma_{100}$ & Normative Consensus & 0.72 & 0.65 & 0.79 & 0.03 & 21.14 & 316.01 & $<.001$ & .46 \\
\hline$\gamma_{200}$ & Distinctive Consensus & 0.19 & 0.17 & 0.21 & 0.01 & 16.03 & 198.41 & $<.001$ & .17 \\
\hline$\gamma_{300}$ & Positivity Bias & 0.04 & 0.01 & 0.08 & 0.02 & 2.38 & 444.75 & 0.018 & .05 \\
\hline$\gamma_{400}$ & P1 Extraversion & 0.01 & -0.01 & 0.04 & 0.01 & 1.07 & 120.4 & 0.285 & .01 \\
\hline$\gamma_{110}$ & $\begin{array}{l}\text { P1 Extraversion X } \\
\text { Normative Consensus }\end{array}$ & -0.20 & -0.30 & -0.09 & 0.05 & -3.79 & 88.68 & $<.001$ & -.09 \\
\hline$\gamma_{210}$ & $\begin{array}{l}\text { P1 Extraversion X } \\
\text { Distinctive Consensus }\end{array}$ & 0.02 & -0.01 & 0.06 & 0.02 & 1.34 & 97.09 & 0.185 & .02 \\
\hline$\gamma_{310}$ & $\begin{array}{l}\text { P1 Extraversion X } \\
\text { Positivity Bias }\end{array}$ & 0.17 & 0.09 & 0.26 & 0.04 & 3.99 & 80.54 & $<.001$ & .14 \\
\hline
\end{tabular}

Note. Term refers to the term in the corresponding equation. Component refers to the SAM component. Est. refers to the unstandardized estimate. CI LL and CI UL refer to the lower and upper limits of the $95 \%$ CI respectively. SE refers to standard error. $d f$ were calculated using the Satterthwaite approximation. Std. Est. refers to the standardized estimates. 
NETWORK APPROACH TO REPUTATIONS: SUPPLEMENT

Table S9

Fixed Effects for P2 Extraversion Moderating Hearsay Consensus (SAM)

\begin{tabular}{|c|c|c|c|c|c|c|c|c|c|}
\hline term & component & Est. & CI LL & CI UL & SE & $t$ & $d f$ & $p$ & $\begin{array}{l}\text { Std. } \\
\text { Est }\end{array}$ \\
\hline$\gamma_{000}$ & intercept & 0.01 & -0.01 & 0.03 & 0.01 & 1.14 & 155.90 & .256 & .00 \\
\hline$\gamma_{100}$ & Normative Consensus & 0.73 & 0.66 & 0.79 & 0.03 & 22.24 & 340.65 & $<.001$ & .46 \\
\hline$\gamma_{200}$ & Distinctive Consensus & 0.19 & 0.16 & 0.21 & 0.01 & 15.68 & 178.94 & $<.001$ & .16 \\
\hline$\gamma_{300}$ & Positivity Bias & 0.03 & 0.00 & 0.06 & 0.02 & 2.23 & 661.08 & .026 & .04 \\
\hline$\gamma_{400}$ & P2 Extraversion & 0.02 & 0.00 & 0.04 & 0.01 & 1.78 & 113.61 & 0.78 & .01 \\
\hline$\gamma_{110}$ & $\begin{array}{l}\text { P2 Extraversion X } \\
\text { Normative Consensus }\end{array}$ & -0.36 & -0.44 & -0.28 & 0.04 & -8.69 & 236.41 & $<.001$ & -.18 \\
\hline$\gamma_{210}$ & $\begin{array}{l}\text { P2 Extraversion X } \\
\text { Distinctive Consensus }\end{array}$ & -0.01 & -.0 .04 & 0.02 & 0.02 & -0.44 & 106.51 & .661 & .00 \\
\hline$\gamma_{310}$ & $\begin{array}{l}\text { P2 Extraversion X } \\
\text { Positivity Bias }\end{array}$ & 0.37 & 0.32 & 0.42 & 0.03 & 14.36 & 92.10 & $<.001$ & .31 \\
\hline
\end{tabular}

Note. Term refers to the term in the corresponding equation. Component refers to the SAM component. Est. refers to the unstandardized estimate. CI LL and CI UL refer to the lower and upper limits of the $95 \%$ CI respectively. SE refers to standard error. $d f$ were calculated using the Satterthwaite approximation. Std. Est. refers to the standardized estimates.

Table S10

Fixed Effects for Target Extraversion Moderating Hearsay Accuracy (SAM)

\begin{tabular}{lllrrrrrrr}
\hline & \multicolumn{1}{c}{ component } & Est. & CI LL & CI UL & \multicolumn{1}{c}{ SE } & \multicolumn{1}{c}{$\boldsymbol{t}$} & $\boldsymbol{d} \boldsymbol{f}$ & \multicolumn{1}{c}{$\boldsymbol{p}$} & $\begin{array}{c}\text { Std. } \\
\text { Est }\end{array}$ \\
\hline$\gamma_{000}$ & intercept & 0.00 & -0.01 & 0.02 & 0.01 & 0.29 & 162.84 & .771 & .00 \\
$\gamma_{100}$ & Normative Accuracy & 0.34 & 0.30 & 0.38 & 0.02 & 15.68 & 227.70 & $<.001$ & .24 \\
$\gamma_{200}$ & Distinctive Accuracy & 0.07 & 0.05 & 0.08 & 0.01 & 7.24 & 214.03 & $<.001$ & .07 \\
$\gamma_{300}$ & Positivity Bias & 0.26 & 0.23 & 0.29 & 0.02 & 16.39 & 588.91 & $<.001$ & .29 \\
$\gamma_{400}$ & Target Extraversion & 0.02 & 0.00 & 0.05 & 0.01 & 2.07 & 104.22 & .041 & .02 \\
$\gamma_{110}$ & Target Extraversion X & -0.01 & -0.07 & 0.05 & 0.03 & -0.32 & 140.07 & .748 & .00 \\
& Normative Accuracy & & & & & & & & \\
$\gamma_{210}$ & Target Extraversion X & 0.00 & -0.03 & 0.03 & 0.01 & 0.03 & 109.76 & .978 & .00 \\
& Distinctive Accuracy & & & & & & & & \\
$\gamma_{310}$ & Target Extraversion X & 0.00 & -0.08 & 0.09 & 0.04 & 0.07 & 108.68 & .947 & .00 \\
& Positivity Bias & & & & & & & & \\
\hline
\end{tabular}

Note. Term refers to the term in the corresponding equation. Component refers to the SAM component. Est. refers to the unstandardized estimate. CI LL and CI UL refer to the lower and upper limits of the $95 \%$ CI respectively. SE refers to standard error. $d f$ were calculated using the Satterthwaite approximation. Std. Est. refers to the standardized estimates. 
NETWORK APPROACH TO REPUTATIONS: SUPPLEMENT

Table S1 1

Fixed Effects for P1 Extraversion Moderating Hearsay Accuracy (SAM)

\begin{tabular}{|c|c|c|c|c|c|c|c|c|c|}
\hline term & component & Est. & CI LL & CI UL & SE & $t$ & $d f$ & $p$ & $\begin{array}{l}\text { Std. } \\
\text { Est }\end{array}$ \\
\hline$\gamma_{000}$ & intercept & 0.00 & -0.01 & 0.02 & 0.01 & 0.27 & 179.80 & .785 & .00 \\
\hline$\gamma_{100}$ & Normative Accuracy & 0.33 & 0.29 & 0.38 & 0.02 & 14.49 & 213.41 & $<.001$ & .23 \\
\hline$\gamma_{200}$ & Distinctive Accuracy & 0.07 & 0.05 & 0.08 & 0.01 & 7.20 & 184.44 & $<.001$ & .07 \\
\hline$\gamma_{300}$ & Positivity Bias & 0.27 & 0.24 & 0.31 & 0.02 & 16.91 & 544.72 & $<.001$ & .30 \\
\hline$\gamma_{400}$ & P1 Extraversion & 0.03 & 0.01 & 0.05 & 0.01 & 2.37 & 117.82 & .020 & .02 \\
\hline$\gamma_{110}$ & $\begin{array}{l}\text { P1 Extraversion X } \\
\text { Normative Accuracy }\end{array}$ & -0.06 & -0.13 & 0.00 & 0.03 & -1.87 & 113.00 & .065 & -.03 \\
\hline$\gamma_{210}$ & $\begin{array}{l}\text { P1 Extraversion X } \\
\text { Distinctive Accuracy }\end{array}$ & 0.00 & -0.03 & 0.03 & 0.01 & -0.10 & 111.03 & .919 & .00 \\
\hline$\gamma_{310}$ & $\begin{array}{l}\text { P1 Extraversion X } \\
\text { Positivity Bias }\end{array}$ & 0.11 & 0.01 & 0.20 & 0.05 & 2.21 & 110.70 & .029 & .08 \\
\hline
\end{tabular}

Note. Term refers to the term in the corresponding equation. Component refers to the SAM component. Est. refers to the unstandardized estimate. CI LL and CI UL refer to the lower and upper limits of the $95 \%$ CI respectively. SE refers to standard error. $d f$ were calculated using the Satterthwaite approximation. Std. Est. refers to the standardized estimates.

Table S12

Fixed Effects for P2 Extraversion Moderating Hearsay Accuracy (SAM)

\begin{tabular}{llrrrrrrrr}
\hline & & \multicolumn{1}{c}{ component } & Est. & CI LL & CI UL & \multicolumn{1}{c}{ SE } & \multicolumn{1}{c}{$\boldsymbol{c}$} & $\boldsymbol{d}$ & \multicolumn{1}{c}{$\begin{array}{c}\text { Std. } \\
\text { term }\end{array}$} \\
\hline$\gamma_{000}$ & intercept & 0.01 & -0.01 & 0.03 & 0.01 & 1.12 & 176.96 & .264 & .00 \\
$\gamma_{100}$ & Normative Accuracy & 0.34 & 0.30 & 0.38 & 0.02 & 14.92 & 202.11 & $<.001$ & .24 \\
$\gamma_{200}$ & Distinctive Accuracy & 0.06 & 0.04 & 0.08 & 0.01 & 7.18 & 171.38 & $<.001$ & .06 \\
$\gamma_{300}$ & Positivity Bias & 0.24 & 0.21 & 0.26 & 0.01 & 16.59 & 626.77 & $<.001$ & .26 \\
$\gamma_{400}$ & P2 Extraversion & 0.03 & 0.00 & 0.05 & 0.01 & 2.40 & 118.23 & .018 & .02 \\
$\gamma_{110}$ & P2 Extraversion X & -0.08 & -0.13 & -0.02 & 0.03 & -2.78 & 94.51 & .007 & -.04 \\
& Normative Accuracy & & & & & & & & \\
$\gamma_{210}$ & P2 Extraversion X & -0.01 & -0.03 & 0.02 & 0.01 & -0.40 & 135.70 & .692 & .00 \\
& Distinctive Accuracy & & & & & & & & \\
$\gamma_{310}$ & P2 Extraversion X & 0.19 & 0.13 & 0.26 & 0.03 & 5.89 & 114.04 & $<.001$ & .16 \\
& Positivity Bias & & & & & & & & \\
\hline
\end{tabular}

Note. Term refers to the term in the corresponding equation. Component refers to the SAM component. Est. refers to the unstandardized estimate. CI LL and CI UL refer to the lower and upper limits of the $95 \%$ CI respectively. SE refers to standard error. $d f$ were calculated using the Satterthwaite approximation. Std. Est. refers to the standardized estimates. 
NETWORK APPROACH TO REPUTATIONS: SUPPLEMENT

Table S13

Fixed Effects for P2 Distress Moderating Hearsay Consensus (SAM)

\begin{tabular}{lllrrrrrrr}
\hline \multicolumn{1}{c}{ component } & Est. & CI LL & CI UL & \multicolumn{1}{c}{ SE } & \multicolumn{1}{c}{$\boldsymbol{c}$} & $\boldsymbol{d}$ & \multicolumn{1}{c}{$\boldsymbol{p}$} & \multicolumn{1}{c}{ Estd. } \\
\hline$\gamma_{000}$ & intercept & 0.00 & -0.02 & 0.01 & 0.01 & -0.37 & 170.59 & .712 & .00 \\
$\gamma_{100}$ & Normative Consensus & 0.75 & 0.68 & 0.82 & 0.04 & 20.92 & 345.33 & $<.001$ & .47 \\
$\gamma_{200}$ & Distinctive Consensus & 0.21 & 0.18 & 0.23 & 0.01 & 15.98 & 154.18 & $<.001$ & .18 \\
$\gamma_{300}$ & Positivity Bias & 0.03 & 0.00 & 0.07 & 0.02 & 1.85 & 608.46 & .065 & .04 \\
$\gamma_{400}$ & P2 Distress & 0.01 & -0.01 & 0.04 & 0.01 & 1.13 & 163.77 & .260 & .01 \\
$\gamma_{110}$ & P2 Distress X & -0.06 & -0.18 & 0.06 & 0.06 & -1.05 & 77.94 & .296 & -.03 \\
& Normative Consensus & & & & & & & & \\
$\gamma_{210}$ & P2 Distress X & 0.03 & 0.00 & 0.07 & 0.02 & 1.79 & 114.01 & .076 & .02 \\
& $\begin{array}{l}\text { Distinctive Consensus } \\
\gamma_{310}\end{array}$ & & & & & & & & \\
& P2 Distress X & 0.04 & -0.06 & 0.13 & 0.05 & 0.80 & 75.73 & .427 & .03 \\
& Positivity Bias & & & & & & & & \\
\hline
\end{tabular}

Note. Term refers to the term in the corresponding equation. Component refers to the SAM component. Est. refers to the unstandardized estimate. CI LL and CI UL refer to the lower and upper limits of the $95 \%$ CI respectively. SE refers to standard error. $d f$ were calculated using the Satterthwaite approximation. Std. Est. refers to the standardized estimates.

Table S14

Fixed Effects for P2 Empathic Concern Moderating Hearsay Consensus (SAM)

\begin{tabular}{|c|c|c|c|c|c|c|c|c|c|}
\hline term & component & Est. & CI LL & CI UL & SE & $t$ & $d f$ & $p$ & $\begin{array}{l}\text { Std. } \\
\text { Est }\end{array}$ \\
\hline$\gamma_{000}$ & intercept & 0.00 & -0.02 & 0.01 & 0.01 & -0.46 & 162.92 & .649 & .00 \\
\hline$\gamma_{100}$ & Normative Consensus & 0.74 & 0.67 & 0.81 & 0.03 & 21.22 & 309.19 & $<.001$ & .47 \\
\hline$\gamma_{200}$ & Distinctive Consensus & 0.21 & 0.19 & 0.24 & 0.01 & 16.21 & 163.95 & $<.001$ & .19 \\
\hline$\gamma_{300}$ & Positivity Bias & 0.04 & 0.01 & 0.08 & 0.02 & 2.30 & 465.10 & .022 & .05 \\
\hline$\gamma_{400}$ & P2 Empathic Concern & 0.00 & -0.03 & 0.03 & 0.02 & -0.14 & 122.38 & .886 & .00 \\
\hline$\gamma_{110}$ & $\begin{array}{l}\text { P2 Empathic Concern } \\
\text { X Normative } \\
\text { Consensus }\end{array}$ & 0.10 & -0.05 & 0.25 & 0.08 & 1.35 & 74.89 & .180 & .03 \\
\hline$\gamma_{210}$ & $\begin{array}{l}\text { P2 Empathic Concern } \\
\text { X Distinctive } \\
\text { Consensus }\end{array}$ & 0.00 & -0.05 & 0.04 & 0.02 & -0.12 & 93.52 & .903 & .00 \\
\hline$\gamma_{310}$ & $\begin{array}{l}\text { P2 Empathic Concern } \\
\text { X Positivity Bias }\end{array}$ & 0.01 & -0.10 & 0.12 & 0.06 & 0.17 & 89.68 & .864 & .01 \\
\hline
\end{tabular}

Note. Term refers to the term in the corresponding equation. Component refers to the SAM component. Est. refers to the unstandardized estimate. CI LL and CI UL refer to the lower and upper limits of the $95 \%$ CI respectively. SE refers to standard error. $d f$ were calculated using the Satterthwaite approximation. Std. Est. refers to the standardized estimates. 
NETWORK APPROACH TO REPUTATIONS: SUPPLEMENT

Table S15

Fixed Effects for P2 Fantasy Moderating Hearsay Consensus (SAM)

\begin{tabular}{lllrrrrrrr}
\hline & \multicolumn{1}{c}{ component } & Est. & CI LL & CI UL & \multicolumn{1}{c}{ SE } & \multicolumn{1}{c}{$\boldsymbol{d}$} & $\boldsymbol{d} \boldsymbol{c}$ & \multicolumn{1}{c}{$\begin{array}{c}\text { Std. } \\
\text { term }\end{array}$} & \multicolumn{1}{c}{ Est } \\
\hline$\gamma_{000}$ & intercept & 0.00 & -0.02 & 0.01 & 0.01 & -0.50 & 159.54 & .618 & .00 \\
$\gamma_{100}$ & Normative Consensus & 0.73 & 0.66 & 0.80 & 0.03 & 20.95 & 318.59 & $<.001$ & .46 \\
$\gamma_{200}$ & Distinctive Consensus & 0.21 & 0.19 & 0.24 & 0.01 & 16.49 & 176.21 & $<.001$ & .19 \\
$\gamma_{300}$ & Positivity Bias & 0.06 & 0.03 & 0.10 & 0.02 & 3.39 & 556.31 & $<.001$ & .07 \\
$\gamma_{400}$ & P2 Fantasy & 0.00 & -0.02 & 0.02 & 0.01 & 0.25 & 106.16 & .806 & .00 \\
$\gamma_{110}$ & P2 Fantasy X & 0.05 & -0.05 & 0.15 & 0.05 & 0.99 & 132.08 & .325 & .02 \\
& Normative Consensus & & & & & & & & \\
$\gamma_{210}$ & P2 Fantasy X & -0.02 & -0.05 & 0.01 & 0.02 & -1.33 & 121.16 & .185 & -.02 \\
& Distinctive Consensus & & & & & & & & \\
$\gamma_{310}$ & P2 Fantasy X & 0.00 & -0.07 & 0.08 & 0.04 & 0.08 & 113.37 & .933 & .00 \\
& Positivity Bias & & & & & & & & \\
\hline
\end{tabular}

Note. Term refers to the term in the corresponding equation. Component refers to the SAM component. Est. refers to the unstandardized estimate. CI LL and CI UL refer to the lower and upper limits of the $95 \%$ CI respectively. SE refers to standard error. $d f$ were calculated using the Satterthwaite approximation. Std. Est. refers to the standardized estimates.

Table S16

Fixed Effects for P2 Perspective Taking Hearsay Consensus (SAM)

\begin{tabular}{|c|c|c|c|c|c|c|c|c|c|}
\hline term & component & Est. & CI LL & CI UL & SE & $t$ & $d f$ & $p$ & $\begin{array}{l}\text { Std. } \\
\text { Est }\end{array}$ \\
\hline$\gamma_{000}$ & intercept & 0 & -0.02 & 0.01 & 0.01 & -0.32 & 215.28 & 0.752 & 0 \\
\hline$\gamma_{100}$ & Normative Consensus & 0.74 & 0.67 & 0.81 & 0.04 & 20.19 & 284.12 & $<.001$ & 0.47 \\
\hline$\gamma_{200}$ & Distinctive Consensus & 0.21 & 0.18 & 0.23 & 0.01 & 16.09 & 139.56 & $<.001$ & 0.19 \\
\hline$\gamma_{300}$ & Positivity Bias & 0.05 & 0.01 & 0.09 & 0.02 & 2.63 & 526.28 & 0.009 & 0.05 \\
\hline$\gamma_{400}$ & P2 Perspective Taking & 0 & -0.02 & 0.03 & 0.01 & 0.14 & 60.6 & 0.892 & 0 \\
\hline$\gamma_{110}$ & $\begin{array}{l}\text { P2 Perspective Taking } \\
\text { X Normative } \\
\text { Consensus }\end{array}$ & 0.01 & -0.12 & 0.14 & 0.07 & 0.17 & 123.68 & 0.866 & 0 \\
\hline$\gamma_{210}$ & $\begin{array}{l}\text { P2 Perspective Taking } \\
\text { X Distinctive } \\
\text { Consensus }\end{array}$ & -0.02 & -0.06 & 0.03 & 0.02 & -0.71 & 86.35 & 0.477 & -0.01 \\
\hline$\gamma_{310}$ & $\begin{array}{l}\text { P2 Perspective Taking } \\
\text { X Positivity Bias }\end{array}$ & 0.06 & -0.05 & 0.16 & 0.05 & 1.08 & 113.07 & 0.282 & 0.04 \\
\hline
\end{tabular}

Note. Term refers to the term in the corresponding equation. Component refers to the SAM component. Est. refers to the unstandardized estimate. CI LL and CI UL refer to the lower and upper limits of the $95 \%$ CI respectively. SE refers to standard error. $d f$ were calculated using the Satterthwaite approximation. Std. Est. refers to the standardized estimates. 
NETWORK APPROACH TO REPUTATIONS: SUPPLEMENT

Table S17

Fixed Effects for P2 Distress Moderating Hearsay Accuracy (SAM)

\begin{tabular}{lllrrrrrrr}
\hline & \multicolumn{1}{c}{ component } & Est. & CI LL & CI UL & \multicolumn{1}{c}{ SE } & \multicolumn{1}{c}{$\boldsymbol{c}$} & $\boldsymbol{d}$ f & \multicolumn{1}{c}{$\boldsymbol{p}$} & \multicolumn{1}{c}{ Estd. } \\
term & \multicolumn{1}{c}{ Est } \\
\hline$\gamma_{000}$ & intercept & 0.00 & -0.02 & 0.02 & 0.01 & 0.08 & 166.71 & .939 & .00 \\
$\gamma_{100}$ & Normative Accuracy & 0.35 & 0.31 & 0.39 & 0.02 & 15.83 & 180.85 & $<.001$ & .24 \\
$\gamma_{200}$ & Distinctive Accuracy & 0.07 & 0.05 & 0.09 & 0.01 & 7.60 & 167.77 & $<.001$ & .07 \\
$\gamma_{300}$ & Positivity Bias & 0.24 & 0.21 & 0.27 & 0.02 & 15.32 & 483.87 & $<.001$ & .26 \\
$\gamma_{400}$ & P2 Distress & 0.01 & -0.01 & 0.04 & 0.01 & 1.07 & 103.49 & .285 & .01 \\
$\gamma_{110}$ & P2 Distress X & -0.04 & -0.10 & 0.03 & 0.03 & -1.08 & 106.09 & .284 & -.02 \\
& Normative Accuracy & & & & & & & & \\
$\gamma_{210}$ & P2 Distress X & 0.00 & -0.03 & 0.03 & 0.02 & -0.08 & 111.69 & .938 & .00 \\
& $\begin{array}{l}\text { Distinctive Accuracy } \\
\gamma_{310}\end{array}$ & & & & & & & & \\
& P2 Distress X & 0.04 & -0.06 & 0.15 & 0.05 & 0.83 & 58.71 & .411 & .03 \\
& Positivity Bias & & & & & & & & \\
\hline
\end{tabular}

Note. Term refers to the term in the corresponding equation. Component refers to the SAM component. Est. refers to the unstandardized estimate. CI LL and CI UL refer to the lower and upper limits of the $95 \%$ CI respectively. SE refers to standard error. $d f$ were calculated using the Satterthwaite approximation. Std. Est. refers to the standardized estimates.

Table S18

Fixed Effects for P2 Empathic Concern Moderating Hearsay Accuracy (SAM)

\begin{tabular}{|c|c|c|c|c|c|c|c|c|c|}
\hline term & component & Est. & CI LL & CI UL & SE & $t$ & $d f$ & $p$ & $\begin{array}{l}\text { Std. } \\
\text { Est }\end{array}$ \\
\hline$\gamma_{000}$ & intercept & 0.00 & -0.02 & 0.02 & 0.01 & -0.22 & 168.52 & .823 & .00 \\
\hline$\gamma_{100}$ & Normative Accuracy & 0.35 & 0.31 & 0.39 & 0.02 & 16.76 & 257.23 & $<.001$ & .24 \\
\hline$\gamma_{200}$ & Distinctive Accuracy & 0.07 & 0.05 & 0.09 & 0.01 & 7.19 & 174.99 & $<.001$ & .07 \\
\hline$\gamma_{300}$ & Positivity Bias & 0.26 & 0.22 & 0.29 & 0.02 & 15.40 & 415.14 & $<.001$ & .28 \\
\hline$\gamma_{400}$ & P2 Empathic Concern & 0.01 & -0.03 & 0.04 & 0.02 & 0.33 & 120.95 & .739 & .00 \\
\hline$\gamma_{110}$ & $\begin{array}{l}\text { P2 Empathic Concern } \\
\text { X Normative } \\
\text { Accuracy }\end{array}$ & 0.07 & -0.01 & 0.15 & 0.04 & 1.61 & 104.32 & .111 & .02 \\
\hline$\gamma_{210}$ & $\begin{array}{l}\text { P2 Empathic Concern } \\
\text { X Distinctive } \\
\text { Accuracy }\end{array}$ & -0.01 & -0.05 & 0.02 & 0.02 & -0.75 & 107.83 & .455 & -.01 \\
\hline$\gamma_{310}$ & $\begin{array}{l}\text { P2 Empathic Concern } \\
\text { X Positivity Bias }\end{array}$ & 0.01 & -0.12 & 0.15 & 0.07 & 0.20 & 82.90 & .844 & .01 \\
\hline
\end{tabular}

Note. Term refers to the term in the corresponding equation. Component refers to the SAM component. Est. refers to the unstandardized estimate. CI LL and CI UL refer to the lower and upper limits of the $95 \%$ CI respectively. SE refers to standard error. $d f$ were calculated using the Satterthwaite approximation. Std. Est. refers to the standardized estimates. 
NETWORK APPROACH TO REPUTATIONS: SUPPLEMENT

Table S19

Fixed Effects for P2 Fantasy Moderating Hearsay Accuracy (SAM)

\begin{tabular}{|c|c|c|c|c|c|c|c|c|c|}
\hline term & component & Est. & CI LL & CI UL & SE & $t$ & $d f$ & $p$ & $\begin{array}{c}\text { Std. } \\
\text { Est }\end{array}$ \\
\hline$\gamma_{000}$ & intercept & 0.00 & -0.02 & 0.01 & 0.01 & -0.34 & 158.52 & .731 & .00 \\
\hline$\gamma_{100}$ & Normative Accuracy & 0.34 & 0.30 & 0.39 & 0.02 & 15.50 & 228.70 & $<.001$ & .24 \\
\hline$\gamma_{200}$ & Distinctive Accuracy & 0.07 & 0.05 & 0.09 & 0.01 & 7.64 & 193.51 & $<.001$ & .07 \\
\hline$\gamma_{300}$ & Positivity Bias & 0.27 & 0.24 & 0.30 & 0.02 & 16.29 & 476.86 & $<.001$ & .29 \\
\hline$\gamma_{400}$ & P2 Fantasy & 0.00 & -0.03 & 0.02 & 0.01 & -0.25 & 95.45 & .800 & .00 \\
\hline$\gamma_{110}$ & $\begin{array}{l}\text { P2 Fantasy X } \\
\text { Normative Accuracy }\end{array}$ & 0.03 & -0.03 & 0.08 & 0.03 & 0.86 & 137.29 & .389 & .01 \\
\hline$\gamma_{210}$ & $\begin{array}{l}\text { P2 Fantasy X } \\
\text { Distinctive Accuracy }\end{array}$ & -0.01 & -0.04 & 0.01 & 0.01 & -1.13 & 118.81 & .260 & -.01 \\
\hline$\gamma_{310}$ & $\begin{array}{l}\text { P2 Fantasy X } \\
\text { Positivity Bias }\end{array}$ & 0.03 & -0.06 & 0.12 & 0.05 & 0.68 & 97.27 & .496 & .03 \\
\hline
\end{tabular}

Note. Term refers to the term in the corresponding equation. Component refers to the SAM component. Est. refers to the unstandardized estimate. CI LL and CI UL refer to the lower and upper limits of the $95 \%$ CI respectively. SE refers to standard error. $d f$ were calculated using the Satterthwaite approximation. Std. Est. refers to the standardized estimates.

Table S20

Fixed Effects for P2 Perspective Taking Moderating Hearsay Accuracy (SAM)

\begin{tabular}{|c|c|c|c|c|c|c|c|c|c|}
\hline term & component & Est. & CI LL & CI UL & SE & $t$ & $d f$ & $p$ & $\begin{array}{l}\text { Std. } \\
\text { Est }\end{array}$ \\
\hline$\gamma_{000}$ & intercept & 0.00 & -0.02 & 0.01 & 0.01 & -0.38 & 167.92 & .705 & .00 \\
\hline$\gamma_{100}$ & Normative Accuracy & 0.34 & 0.30 & 0.38 & 0.02 & 15.64 & 205.97 & $<.001$ & .24 \\
\hline$\gamma_{200}$ & Distinctive Accuracy & 0.07 & 0.05 & 0.09 & 0.01 & 7.48 & 192.97 & $<.001$ & .07 \\
\hline$\gamma_{300}$ & Positivity Bias & 0.27 & 0.23 & 0.30 & 0.02 & 15.58 & 405.59 & $<.001$ & .29 \\
\hline$\gamma_{400}$ & P2 Perspective Taking & 0.00 & -0.03 & 0.02 & 0.01 & -0.24 & 130.08 & .809 & .00 \\
\hline$\gamma_{110}$ & $\begin{array}{l}\text { P2 Perspective Taking } \\
\text { X Normative } \\
\text { Accuracy }\end{array}$ & 0.05 & -0.02 & 0.13 & 0.04 & 1.40 & 116.93 & .164 & .02 \\
\hline$\gamma_{210}$ & $\begin{array}{l}\text { P2 Perspective Taking } \\
\text { X Distinctive } \\
\text { Accuracy }\end{array}$ & 0.01 & -0.02 & 0.04 & 0.02 & 0.49 & 99.18 & .624 & .00 \\
\hline$\gamma_{310}$ & $\begin{array}{l}\text { P2 Perspective Taking } \\
\text { X Positivity Bias }\end{array}$ & 0.04 & -0.08 & 0.17 & 0.06 & 0.72 & 98.61 & .476 & .03 \\
\hline
\end{tabular}

Note. Term refers to the term in the corresponding equation. Component refers to the SAM component. Est. refers to the unstandardized estimate. CI LL and CI UL refer to the lower and upper limits of the $95 \%$ CI respectively. SE refers to standard error. $d f$ were calculated using the Satterthwaite approximation. Std. Est. refers to the standardized estimates. 
NETWORK APPROACH TO REPUTATIONS: SUPPLEMENT

Table S21

Random Effects for Target Extraversion Moderating Hearsay Consensus (SAM)

\begin{tabular}{|c|c|c|c|}
\hline source & type & term & Est. \\
\hline triad:group & variance & Intercept & 0.00 \\
\hline triad:group & variance & Normative Consensus & 0.11 \\
\hline triad:group & variance & Distinctive Consensus & 0.02 \\
\hline triad:group & variance & Target Extraversion & 0.00 \\
\hline triad:group & variance & Normative Consensus X Target Extraversion & 0.12 \\
\hline triad:group & variance & Distinctive Consensus X Target Extraversion & 0.00 \\
\hline triad:group & variance & Target Extraversion X Positivity Bias & 0.27 \\
\hline triad:group & covariance & Intercept, Normative Consensus & -0.01 \\
\hline triad:group & covariance & Intercept, Distinctive Consensus & 0.00 \\
\hline triad:group & covariance & Intercept, Target Extraversion & 0.00 \\
\hline triad:group & covariance & Intercept, Normative Consensus X Target Extraversion & 0.00 \\
\hline triad:group & covariance & Intercept, Distinctive Consensus X Target Extraversion & 0.00 \\
\hline triad:group & covariance & Intercept, Target Extraversion X Positivity Bias & 0.00 \\
\hline triad:group & covariance & Normative Consensus, Distinctive Consensus & 0.00 \\
\hline triad:group & covariance & Normative Consensus, Target Extraversion & 0.00 \\
\hline triad:group & covariance & $\begin{array}{l}\text { Normative Consensus, Normative Consensus X Target } \\
\text { Extraversion }\end{array}$ & 0.01 \\
\hline triad:group & covariance & $\begin{array}{l}\text { Normative Consensus, Distinctive Consensus X Target } \\
\text { Extraversion }\end{array}$ & 0.00 \\
\hline triad:group & covariance & $\begin{array}{l}\text { Normative Consensus, Target Extraversion X Positivity } \\
\text { Bias }\end{array}$ & 0.00 \\
\hline triad:group & covariance & Distinctive Consensus, Target Extraversion & 0.00 \\
\hline
\end{tabular}




\begin{tabular}{|c|c|c|c|}
\hline source & type & term & Est. \\
\hline triad:group & covariance & $\begin{array}{l}\text { Distinctive Consensus, Normative Consensus X Target } \\
\text { Extraversion }\end{array}$ & -0.02 \\
\hline triad:group & covariance & $\begin{array}{l}\text { Distinctive Consensus, Distinctive Consensus X Target } \\
\text { Extraversion }\end{array}$ & 0.00 \\
\hline triad:group & covariance & $\begin{array}{l}\text { Distinctive Consensus, Target Extraversion X Positivity } \\
\text { Bias }\end{array}$ & 0.01 \\
\hline triad:group & covariance & $\begin{array}{l}\text { Target Extraversion, Normative Consensus X Target } \\
\text { Extraversion }\end{array}$ & 0.00 \\
\hline triad:group & covariance & $\begin{array}{l}\text { Target Extraversion, Distinctive Consensus X Target } \\
\text { Extraversion }\end{array}$ & 0.00 \\
\hline triad:group & covariance & $\begin{array}{l}\text { Target Extraversion, Target Extraversion X Positivity } \\
\text { Bias }\end{array}$ & -0.01 \\
\hline triad:group & covariance & $\begin{array}{l}\text { Normative Consensus X Target Extraversion, } \\
\text { Distinctive Consensus X Target Extraversion }\end{array}$ & 0.01 \\
\hline triad:group & covariance & $\begin{array}{l}\text { Normative Consensus X Target Extraversion, Target } \\
\text { Extraversion X Positivity Bias }\end{array}$ & -0.18 \\
\hline triad:group & covariance & $\begin{array}{l}\text { Distinctive Consensus X Target Extraversion, Target } \\
\text { Extraversion X Positivity Bias }\end{array}$ & -0.01 \\
\hline group & variance & Intercept & 0.00 \\
\hline group & variance & Normative Consensus & 0.03 \\
\hline group & variance & Distinctive Consensus & 0.00 \\
\hline group & variance & Target Extraversion & 0.00 \\
\hline group & variance & Normative Consensus X Target Extraversion & 0.01 \\
\hline group & variance & Distinctive Consensus X Target Extraversion & 0.00 \\
\hline group & variance & Target Extraversion X Positivity Bias & 0.00 \\
\hline group & covariance & Intercept, Normative Consensus & 0.00 \\
\hline group & covariance & Intercept, Distinctive Consensus & 0.00 \\
\hline group & covariance & Intercept, Target Extraversion & 0.00 \\
\hline
\end{tabular}




\begin{tabular}{|c|c|c|c|}
\hline source & type & term & Est. \\
\hline group & covariance & Intercept, Normative Consensus X Target Extraversion & 0.00 \\
\hline group & covariance & Intercept, Distinctive Consensus X Target Extraversion & 0.00 \\
\hline group & covariance & Intercept, Target Extraversion X Positivity Bias & 0.00 \\
\hline group & covariance & Normative Consensus, Distinctive Consensus & 0.01 \\
\hline group & covariance & Normative Consensus, Target Extraversion & 0.01 \\
\hline group & covariance & $\begin{array}{l}\text { Normative Consensus, Normative Consensus X Target } \\
\text { Extraversion }\end{array}$ & 0.00 \\
\hline group & covariance & $\begin{array}{l}\text { Normative Consensus, Distinctive Consensus X Target } \\
\text { Extraversion }\end{array}$ & 0.00 \\
\hline group & covariance & $\begin{array}{l}\text { Normative Consensus, Target Extraversion X Positivity } \\
\text { Bias }\end{array}$ & -0.01 \\
\hline group & covariance & Distinctive Consensus, Target Extraversion & 0.00 \\
\hline group & covariance & $\begin{array}{l}\text { Distinctive Consensus, Normative Consensus X Target } \\
\text { Extraversion }\end{array}$ & 0.00 \\
\hline group & covariance & $\begin{array}{l}\text { Distinctive Consensus, Distinctive Consensus X Target } \\
\text { Extraversion }\end{array}$ & 0.00 \\
\hline group & covariance & $\begin{array}{l}\text { Distinctive Consensus, Target Extraversion X Positivity } \\
\text { Bias }\end{array}$ & 0.00 \\
\hline group & covariance & $\begin{array}{l}\text { Target Extraversion, Normative Consensus X Target } \\
\text { Extraversion }\end{array}$ & 0.00 \\
\hline group & covariance & $\begin{array}{l}\text { Target Extraversion, Distinctive Consensus X Target } \\
\text { Extraversion }\end{array}$ & 0.00 \\
\hline group & covariance & $\begin{array}{l}\text { Target Extraversion, Target Extraversion X Positivity } \\
\text { Bias }\end{array}$ & 0.00 \\
\hline group & covariance & $\begin{array}{l}\text { Normative Consensus X Target Extraversion, } \\
\text { Distinctive Consensus X Target Extraversion }\end{array}$ & 0.00 \\
\hline group & covariance & $\begin{array}{l}\text { Normative Consensus X Target Extraversion, Target } \\
\text { Extraversion X Positivity Bias }\end{array}$ & 0.00 \\
\hline
\end{tabular}


NETWORK APPROACH TO REPUTATIONS: SUPPLEMENT

\begin{tabular}{llll}
\hline source & type & term & Est. \\
\hline group & covariance & $\begin{array}{l}\text { Distinctive Consensus X Target Extraversion, Target } \\
\text { Extraversion X Positivity Bias }\end{array}$ & 0.00 \\
Residual & & & 0.69 \\
\hline
\end{tabular}


NETWORK APPROACH TO REPUTATIONS: SUPPLEMENT

Table S22

Random Effects for P1 Extraversion Moderating Hearsay Consensus (SAM)

\begin{tabular}{|c|c|c|c|}
\hline source & type & term & Est. \\
\hline triad:group & variance & Intercept & 0.00 \\
\hline triad:group & variance & Normative Consensus & 0.12 \\
\hline triad:group & variance & Distinctive Consensus & 0.01 \\
\hline triad:group & variance & P1 Extraversion & 0.00 \\
\hline triad:group & variance & Normative Consensus X P1 Extraversion & 0.10 \\
\hline triad:group & variance & Distinctive Consensus X P1 Extraversion & 0.01 \\
\hline triad:group & variance & P1 Extraversion X Positivity Bias & 0.27 \\
\hline triad:group & covariance & Intercept, Normative Consensus & -0.01 \\
\hline triad:group & covariance & Intercept, Distinctive Consensus & 0.00 \\
\hline triad:group & covariance & Intercept, P1 Extraversion & 0.00 \\
\hline triad:group & covariance & Intercept, Normative Consensus X P1 Extraversion & 0.00 \\
\hline triad:group & covariance & Intercept, Distinctive Consensus X P1 Extraversion & 0.00 \\
\hline triad:group & covariance & Intercept, P1 Extraversion X Positivity Bias & 0.00 \\
\hline triad:group & covariance & Normative Consensus, Distinctive Consensus & -0.01 \\
\hline triad:group & covariance & Normative Consensus, P1 Extraversion & 0.01 \\
\hline triad:group & covariance & $\begin{array}{l}\text { Normative Consensus, Normative Consensus X P1 } \\
\text { Extraversion }\end{array}$ & 0.00 \\
\hline triad:group & covariance & $\begin{array}{l}\text { Normative Consensus, Distinctive Consensus X P1 } \\
\text { Extraversion }\end{array}$ & -0.01 \\
\hline triad:group & covariance & Normative Consensus, P1 Extraversion X Positivity Bias & 0.00 \\
\hline triad:group & covariance & Distinctive Consensus, P1 Extraversion & 0.00 \\
\hline triad:group & covariance & $\begin{array}{l}\text { Distinctive Consensus, Normative Consensus X P1 } \\
\text { Extraversion }\end{array}$ & -0.01 \\
\hline
\end{tabular}




\begin{tabular}{|c|c|c|c|}
\hline source & type & term & Est. \\
\hline triad:group & covariance & $\begin{array}{l}\text { Distinctive Consensus, Distinctive Consensus X P1 } \\
\text { Extraversion }\end{array}$ & -0.01 \\
\hline triad:group & covariance & Distinctive Consensus, P1 Extraversion X Positivity Bias & 0.01 \\
\hline triad:group & covariance & P1 Extraversion, Normative Consensus X P1 Extraversion & 0.01 \\
\hline triad:group & covariance & P1 Extraversion, Distinctive Consensus X P1 Extraversion & -0.01 \\
\hline triad:group & covariance & P1 Extraversion, P1 Extraversion X Positivity Bias & -0.02 \\
\hline triad:group & covariance & $\begin{array}{l}\text { Normative Consensus X P1 Extraversion, Distinctive } \\
\text { Consensus X P1 Extraversion }\end{array}$ & 0.01 \\
\hline triad:group & covariance & $\begin{array}{l}\text { Normative Consensus X P1 Extraversion, P1 Extraversion } \\
\text { X Positivity Bias }\end{array}$ & -0.15 \\
\hline triad:group & covariance & $\begin{array}{l}\text { Distinctive Consensus X P1 Extraversion, P1 Extraversion } \\
\text { X Positivity Bias }\end{array}$ & 0.00 \\
\hline group & variance & Intercept & 0.00 \\
\hline group & variance & Normative Consensus & 0.03 \\
\hline group & variance & Distinctive Consensus & 0.00 \\
\hline group & variance & P1 Extraversion & 0.00 \\
\hline group & variance & Normative Consensus X P1 Extraversion & 0.03 \\
\hline group & variance & Distinctive Consensus X P1 Extraversion & 0.00 \\
\hline group & variance & P1 Extraversion X Positivity Bias & 0.01 \\
\hline group & covariance & Intercept, Normative Consensus & 0.00 \\
\hline group & covariance & Intercept, Distinctive Consensus & 0.00 \\
\hline group & covariance & Intercept, P1 Extraversion & 0.00 \\
\hline group & covariance & Intercept, Normative Consensus X P1 Extraversion & 0.01 \\
\hline group & covariance & Intercept, Distinctive Consensus X P1 Extraversion & 0.00 \\
\hline group & covariance & Intercept, P1 Extraversion X Positivity Bias & 0.00 \\
\hline
\end{tabular}


NETWORK APPROACH TO REPUTATIONS: SUPPLEMENT

\begin{tabular}{|c|c|c|c|}
\hline source & type & term & Est. \\
\hline group & covariance & Normative Consensus, Distinctive Consensus & 0.01 \\
\hline group & covariance & Normative Consensus, P1 Extraversion & 0.00 \\
\hline group & covariance & $\begin{array}{l}\text { Normative Consensus, Normative Consensus X P1 } \\
\text { Extraversion }\end{array}$ & -0.02 \\
\hline group & covariance & $\begin{array}{l}\text { Normative Consensus, Distinctive Consensus X P1 } \\
\text { Extraversion }\end{array}$ & 0.00 \\
\hline group & covariance & Normative Consensus, P1 Extraversion X Positivity Bias & 0.01 \\
\hline group & covariance & Distinctive Consensus, P1 Extraversion & 0.00 \\
\hline group & covariance & $\begin{array}{l}\text { Distinctive Consensus, Normative Consensus X P1 } \\
\text { Extraversion }\end{array}$ & 0.00 \\
\hline group & covariance & $\begin{array}{l}\text { Distinctive Consensus, Distinctive Consensus X P1 } \\
\text { Extraversion }\end{array}$ & 0.00 \\
\hline group & covariance & Distinctive Consensus, P1 Extraversion X Positivity Bias & 0.00 \\
\hline group & covariance & P1 Extraversion, Normative Consensus X P1 Extraversion & -0.01 \\
\hline group & covariance & P1 Extraversion, Distinctive Consensus X P1 Extraversion & 0.00 \\
\hline group & covariance & P1 Extraversion, P1 Extraversion X Positivity Bias & 0.00 \\
\hline group & covariance & $\begin{array}{l}\text { Normative Consensus X P1 Extraversion, Distinctive } \\
\text { Consensus X P1 Extraversion }\end{array}$ & 0.00 \\
\hline group & covariance & $\begin{array}{l}\text { Normative Consensus X P1 Extraversion, P1 Extraversion } \\
\text { X Positivity Bias }\end{array}$ & -0.02 \\
\hline group & covariance & $\begin{array}{l}\text { Distinctive Consensus X P1 Extraversion, P1 Extraversion } \\
\text { X Positivity Bias }\end{array}$ & 0.00 \\
\hline Residual & & & 0.69 \\
\hline
\end{tabular}


NETWORK APPROACH TO REPUTATIONS: SUPPLEMENT

Table S23

Random Effects for P2 Extraversion Moderating Hearsay Consensus (SAM)

\begin{tabular}{|c|c|c|c|}
\hline source & type & term & Est. \\
\hline triad:group & variance & Intercept & 0.00 \\
\hline triad:group & variance & Normative Consensus & 0.11 \\
\hline triad:group & variance & Distinctive Consensus & 0.01 \\
\hline triad:group & variance & P2 Extraversion & 0.00 \\
\hline triad:group & variance & Normative Consensus X P2 Extraversion & 0.00 \\
\hline triad:group & variance & Distinctive Consensus X P2 Extraversion & 0.00 \\
\hline triad:group & variance & P2 Extraversion X Positivity Bias & 0.03 \\
\hline triad:group & covariance & Intercept, Normative Consensus & -0.01 \\
\hline triad:group & covariance & Intercept, Distinctive Consensus & 0.00 \\
\hline triad:group & covariance & Intercept, P2 Extraversion & 0.00 \\
\hline triad:group & covariance & Intercept, Normative Consensus X P2 Extraversion & 0.00 \\
\hline triad:group & covariance & Intercept, Distinctive Consensus X P2 Extraversion & 0.00 \\
\hline triad:group & covariance & Intercept, P2 Extraversion X Positivity Bias & 0.00 \\
\hline triad:group & covariance & Normative Consensus, Distinctive Consensus & -0.01 \\
\hline triad:group & covariance & Normative Consensus, P2 Extraversion & 0.00 \\
\hline triad:group & covariance & $\begin{array}{l}\text { Normative Consensus, Normative Consensus X P2 } \\
\text { Extraversion }\end{array}$ & -0.01 \\
\hline triad:group & covariance & $\begin{array}{l}\text { Normative Consensus, Distinctive Consensus X P2 } \\
\text { Extraversion }\end{array}$ & -0.01 \\
\hline triad:group & covariance & Normative Consensus, P2 Extraversion X Positivity Bias & 0.01 \\
\hline triad:group & covariance & Distinctive Consensus, P2 Extraversion & 0.00 \\
\hline triad:group & covariance & $\begin{array}{l}\text { Distinctive Consensus, Normative Consensus X P2 } \\
\text { Extraversion }\end{array}$ & 0.00 \\
\hline
\end{tabular}




\begin{tabular}{|c|c|c|c|}
\hline source & type & term & Est. \\
\hline triad:group & covariance & $\begin{array}{l}\text { Distinctive Consensus, Distinctive Consensus X P2 } \\
\text { Extraversion }\end{array}$ & 0.00 \\
\hline triad:group & covariance & Distinctive Consensus, P2 Extraversion X Positivity Bias & -0.02 \\
\hline triad:group & covariance & P2 Extraversion, Normative Consensus X P2 Extraversion & 0.00 \\
\hline triad:group & covariance & P2 Extraversion, Distinctive Consensus X P2 Extraversion & 0.00 \\
\hline triad:group & covariance & P2 Extraversion, P2 Extraversion X Positivity Bias & -0.01 \\
\hline triad:group & covariance & $\begin{array}{l}\text { Normative Consensus X P2 Extraversion, Distinctive } \\
\text { Consensus X P2 Extraversion }\end{array}$ & 0.00 \\
\hline triad:group & covariance & $\begin{array}{l}\text { Normative Consensus X P2 Extraversion, P2 Extraversion } \\
\text { X Positivity Bias }\end{array}$ & 0.00 \\
\hline triad:group & covariance & $\begin{array}{l}\text { Distinctive Consensus X P2 Extraversion, P2 Extraversion } \\
\text { X Positivity Bias }\end{array}$ & 0.01 \\
\hline group & variance & Intercept & 0.00 \\
\hline group & variance & Normative Consensus & 0.03 \\
\hline group & variance & Distinctive Consensus & 0.00 \\
\hline group & variance & P2 Extraversion & 0.00 \\
\hline group & variance & Normative Consensus X P2 Extraversion & 0.01 \\
\hline group & variance & Distinctive Consensus X P2 Extraversion & 0.01 \\
\hline group & variance & P2 Extraversion X Positivity Bias & 0.03 \\
\hline group & covariance & Intercept, Normative Consensus & 0.00 \\
\hline group & covariance & Intercept, Distinctive Consensus & 0.00 \\
\hline group & covariance & Intercept, P2 Extraversion & 0.00 \\
\hline group & covariance & Intercept, Normative Consensus X P2 Extraversion & 0.00 \\
\hline group & covariance & Intercept, Distinctive Consensus X P2 Extraversion & 0.00 \\
\hline group & covariance & Intercept, P2 Extraversion X Positivity Bias & 0.00 \\
\hline
\end{tabular}


NETWORK APPROACH TO REPUTATIONS: SUPPLEMENT

\begin{tabular}{|c|c|c|c|}
\hline source & type & term & Est. \\
\hline group & covariance & Normative Consensus, Distinctive Consensus & 0.01 \\
\hline group & covariance & Normative Consensus, P2 Extraversion & 0.00 \\
\hline group & covariance & $\begin{array}{l}\text { Normative Consensus, Normative Consensus X P2 } \\
\text { Extraversion }\end{array}$ & 0.00 \\
\hline group & covariance & $\begin{array}{l}\text { Normative Consensus, Distinctive Consensus X P2 } \\
\text { Extraversion }\end{array}$ & 0.01 \\
\hline group & covariance & Normative Consensus, P2 Extraversion X Positivity Bias & 0.00 \\
\hline group & covariance & Distinctive Consensus, P2 Extraversion & 0.00 \\
\hline group & covariance & $\begin{array}{l}\text { Distinctive Consensus, Normative Consensus X P2 } \\
\text { Extraversion }\end{array}$ & 0.00 \\
\hline group & covariance & $\begin{array}{l}\text { Distinctive Consensus, Distinctive Consensus X P2 } \\
\text { Extraversion }\end{array}$ & 0.00 \\
\hline group & covariance & Distinctive Consensus, P2 Extraversion X Positivity Bias & -0.01 \\
\hline group & covariance & P2 Extraversion, Normative Consensus X P2 Extraversion & 0.00 \\
\hline group & covariance & P2 Extraversion, Distinctive Consensus X P2 Extraversion & 0.00 \\
\hline group & covariance & P2 Extraversion, P2 Extraversion X Positivity Bias & 0.00 \\
\hline group & covariance & $\begin{array}{l}\text { Normative Consensus X P2 Extraversion, Distinctive } \\
\text { Consensus X P2 Extraversion }\end{array}$ & 0.00 \\
\hline group & covariance & $\begin{array}{l}\text { Normative Consensus X P2 Extraversion, P2 Extraversion } \\
\text { X Positivity Bias }\end{array}$ & -0.01 \\
\hline group & covariance & $\begin{array}{l}\text { Distinctive Consensus X P2 Extraversion, P2 Extraversion } \\
\text { X Positivity Bias }\end{array}$ & 0.01 \\
\hline Residual & & & 0.69 \\
\hline
\end{tabular}


NETWORK APPROACH TO REPUTATIONS: SUPPLEMENT

Table S24

Random Effects for Target Extraversion Moderating Hearsay Accuracy (SAM)

\begin{tabular}{|c|c|c|c|}
\hline source & type & term & Est. \\
\hline triad:group & variance & Intercept & 0.00 \\
\hline triad:group & variance & Normative Accuracy & 0.05 \\
\hline triad:group & variance & Distinctive Accuracy & 0.01 \\
\hline triad:group & variance & Target Extraversion & 0.00 \\
\hline triad:group & variance & Normative Accuracy X Target Extraversion & 0.02 \\
\hline triad:group & variance & Distinctive Accuracy X Target Extraversion & 0.01 \\
\hline triad:group & variance & Target Extraversion X Positivity Bias & 0.36 \\
\hline triad:group & covariance & Intercept, Normative Accuracy & -0.01 \\
\hline triad:group & covariance & Intercept, Distinctive Accuracy & 0.00 \\
\hline triad:group & covariance & Intercept, Target Extraversion & 0.00 \\
\hline triad:group & covariance & Intercept, Normative Accuracy X Target Extraversion & 0.00 \\
\hline triad:group & covariance & Intercept, Distinctive Accuracy X Target Extraversion & 0.00 \\
\hline triad:group & covariance & Intercept, Target Extraversion X Positivity Bias & 0.00 \\
\hline triad:group & covariance & Normative Accuracy, Distinctive Accuracy & 0.01 \\
\hline triad:group & covariance & Normative Accuracy, Target Extraversion & 0.00 \\
\hline triad:group & covariance & $\begin{array}{l}\text { Normative Accuracy, Normative Accuracy X Target } \\
\text { Extraversion }\end{array}$ & 0.00 \\
\hline triad:group & covariance & $\begin{array}{l}\text { Normative Accuracy, Distinctive Accuracy X Target } \\
\text { Extraversion }\end{array}$ & -0.01 \\
\hline triad:group & covariance & $\begin{array}{l}\text { Normative Accuracy, Target Extraversion X Positivity } \\
\text { Bias }\end{array}$ & -0.02 \\
\hline triad:group & covariance & Distinctive Accuracy, Target Extraversion & 0.00 \\
\hline triad:group & covariance & $\begin{array}{l}\text { Distinctive Accuracy, Normative Accuracy X Target } \\
\text { Extraversion }\end{array}$ & 0.00 \\
\hline
\end{tabular}




\begin{tabular}{|c|c|c|c|}
\hline source & type & term & Est. \\
\hline triad:group & covariance & $\begin{array}{l}\text { Distinctive Accuracy, Distinctive Accuracy X Target } \\
\text { Extraversion }\end{array}$ & 0.00 \\
\hline triad:group & covariance & $\begin{array}{l}\text { Distinctive Accuracy, Target Extraversion X Positivity } \\
\text { Bias }\end{array}$ & 0.01 \\
\hline triad:group & covariance & $\begin{array}{l}\text { Target Extraversion, Normative Accuracy X Target } \\
\text { Extraversion }\end{array}$ & 0.01 \\
\hline triad:group & covariance & $\begin{array}{l}\text { Target Extraversion, Distinctive Accuracy X Target } \\
\text { Extraversion }\end{array}$ & 0.00 \\
\hline triad:group & covariance & Target Extraversion, Target Extraversion X Positivity Bias & -0.04 \\
\hline triad:group & covariance & $\begin{array}{l}\text { Normative Accuracy X Target Extraversion, Distinctive } \\
\text { Accuracy X Target Extraversion }\end{array}$ & 0.00 \\
\hline triad:group & covariance & $\begin{array}{l}\text { Normative Accuracy X Target Extraversion, Target } \\
\text { Extraversion X Positivity Bias }\end{array}$ & -0.09 \\
\hline triad:group & covariance & $\begin{array}{l}\text { Distinctive Accuracy X Target Extraversion, Target } \\
\text { Extraversion X Positivity Bias }\end{array}$ & 0.00 \\
\hline group & variance & Intercept & 0.00 \\
\hline group & variance & Normative Accuracy & 0.01 \\
\hline group & variance & Distinctive Accuracy & 0.00 \\
\hline group & variance & Target Extraversion & 0.00 \\
\hline group & variance & Normative Accuracy X Target Extraversion & 0.00 \\
\hline group & variance & Distinctive Accuracy X Target Extraversion & 0.00 \\
\hline group & variance & Target Extraversion X Positivity Bias & 0.01 \\
\hline group & covariance & Intercept, Normative Accuracy & 0.00 \\
\hline group & covariance & Intercept, Distinctive Accuracy & 0.00 \\
\hline group & covariance & Intercept, Target Extraversion & 0.00 \\
\hline group & covariance & Intercept, Normative Accuracy X Target Extraversion & 0.00 \\
\hline group & covariance & Intercept, Distinctive Accuracy X Target Extraversion & 0.00 \\
\hline
\end{tabular}


NETWORK APPROACH TO REPUTATIONS: SUPPLEMENT

\begin{tabular}{|c|c|c|c|}
\hline source & type & term & Est \\
\hline group & covariance & Intercept, Target Extraversion X Positivity Bias & 0.00 \\
\hline group & covariance & Normative Accuracy, Distinctive Accuracy & 0.00 \\
\hline group & covariance & Normative Accuracy, Target Extraversion & 0.00 \\
\hline group & covariance & $\begin{array}{l}\text { Normative Accuracy, Normative Accuracy X Target } \\
\text { Extraversion }\end{array}$ & 0.00 \\
\hline group & covariance & $\begin{array}{l}\text { Normative Accuracy, Distinctive Accuracy X Target } \\
\text { Extraversion }\end{array}$ & 0.00 \\
\hline group & covariance & $\begin{array}{l}\text { Normative Accuracy, Target Extraversion X Positivity } \\
\text { Bias }\end{array}$ & 0.01 \\
\hline group & covariance & Distinctive Accuracy, Target Extraversion & 0.00 \\
\hline group & covariance & $\begin{array}{l}\text { Distinctive Accuracy, Normative Accuracy X Target } \\
\text { Extraversion }\end{array}$ & 0.00 \\
\hline group & covariance & $\begin{array}{l}\text { Distinctive Accuracy, Distinctive Accuracy X Target } \\
\text { Extraversion }\end{array}$ & 0.00 \\
\hline group & covariance & $\begin{array}{l}\text { Distinctive Accuracy, Target Extraversion X Positivity } \\
\text { Bias }\end{array}$ & 0.00 \\
\hline group & covariance & $\begin{array}{l}\text { Target Extraversion, Normative Accuracy X Target } \\
\text { Extraversion }\end{array}$ & 0.00 \\
\hline group & covariance & $\begin{array}{l}\text { Target Extraversion, Distinctive Accuracy X Target } \\
\text { Extraversion }\end{array}$ & 0.00 \\
\hline group & covariance & Target Extraversion, Target Extraversion X Positivity Bias & 0.00 \\
\hline group & covariance & $\begin{array}{l}\text { Normative Accuracy X Target Extraversion, Distinctive } \\
\text { Accuracy X Target Extraversion }\end{array}$ & 0.00 \\
\hline group & covariance & $\begin{array}{l}\text { Normative Accuracy X Target Extraversion, Target } \\
\text { Extraversion X Positivity Bias }\end{array}$ & 0.00 \\
\hline group & covariance & $\begin{array}{l}\text { Distinctive Accuracy X Target Extraversion, Target } \\
\text { Extraversion X Positivity Bias }\end{array}$ & 0.00 \\
\hline Residual & variance & Observation & 0.76 \\
\hline
\end{tabular}


NETWORK APPROACH TO REPUTATIONS: SUPPLEMENT

Table S25

Random Effects for P1 Extraversion Moderating Hearsay Accuracy (SAM)

\begin{tabular}{|c|c|c|c|}
\hline source & type & term & Est. \\
\hline triad:group & variance & Intercept & 0.00 \\
\hline triad:group & variance & Normative Accuracy & 0.05 \\
\hline triad:group & variance & Distinctive Accuracy & 0.01 \\
\hline triad:group & variance & P1 Extraversion & 0.01 \\
\hline triad:group & variance & Normative Accuracy X P1 Extraversion & 0.04 \\
\hline triad:group & variance & Distinctive Accuracy X P1 Extraversion & 0.01 \\
\hline triad:group & variance & P1 Extraversion X Positivity Bias & 0.41 \\
\hline triad:group & covariance & Intercept, Normative Accuracy & 0.00 \\
\hline triad:group & covariance & Intercept, Distinctive Accuracy & 0.00 \\
\hline triad:group & covariance & Intercept, P1 Extraversion & 0.00 \\
\hline triad:group & covariance & Intercept, Normative Accuracy X P1 Extraversion & 0.00 \\
\hline triad:group & covariance & Intercept, Distinctive Accuracy X P1 Extraversion & 0.00 \\
\hline triad:group & covariance & Intercept, P1 Extraversion X Positivity Bias & 0.00 \\
\hline triad:group & covariance & Normative Accuracy, Distinctive Accuracy & 0.01 \\
\hline triad:group & covariance & Normative Accuracy, P1 Extraversion & 0.00 \\
\hline triad:group & covariance & $\begin{array}{l}\text { Normative Accuracy, Normative Accuracy X P1 } \\
\text { Extraversion }\end{array}$ & 0.00 \\
\hline triad:group & covariance & $\begin{array}{l}\text { Normative Accuracy, Distinctive Accuracy X P1 } \\
\text { Extraversion }\end{array}$ & 0.00 \\
\hline triad:group & covariance & Normative Accuracy, P1 Extraversion X Positivity Bias & -0.01 \\
\hline triad:group & covariance & Distinctive Accuracy, P1 Extraversion & 0.00 \\
\hline triad:group & covariance & $\begin{array}{l}\text { Distinctive Accuracy, Normative Accuracy X P1 } \\
\text { Extraversion }\end{array}$ & -0.01 \\
\hline
\end{tabular}




\begin{tabular}{|c|c|c|c|}
\hline source & type & term & Est. \\
\hline triad:group & covariance & $\begin{array}{l}\text { Distinctive Accuracy, Distinctive Accuracy X P1 } \\
\text { Extraversion }\end{array}$ & 0.00 \\
\hline triad:group & covariance & Distinctive Accuracy, P1 Extraversion X Positivity Bias & 0.01 \\
\hline triad:group & covariance & P1 Extraversion, Normative Accuracy X P1 Extraversion & 0.01 \\
\hline triad:group & covariance & P1 Extraversion, Distinctive Accuracy X P1 Extraversion & 0.00 \\
\hline triad:group & covariance & P1 Extraversion, P1 Extraversion X Positivity Bias & -0.05 \\
\hline triad:group & covariance & $\begin{array}{l}\text { Normative Accuracy X P1 Extraversion, Distinctive } \\
\text { Accuracy X P1 Extraversion }\end{array}$ & 0.01 \\
\hline triad:group & covariance & $\begin{array}{l}\text { Normative Accuracy X P1 Extraversion, P1 Extraversion } \\
\text { X Positivity Bias }\end{array}$ & -0.11 \\
\hline triad:group & covariance & $\begin{array}{l}\text { Distinctive Accuracy X P1 Extraversion, P1 Extraversion } \\
\text { X Positivity Bias }\end{array}$ & -0.01 \\
\hline group & variance & Intercept & 0.00 \\
\hline group & variance & Normative Accuracy & 0.02 \\
\hline group & variance & Distinctive Accuracy & 0.00 \\
\hline group & variance & P1 Extraversion & 0.00 \\
\hline group & variance & Normative Accuracy X P1 Extraversion & 0.01 \\
\hline group & variance & Distinctive Accuracy X P1 Extraversion & 0.00 \\
\hline group & variance & P1 Extraversion X Positivity Bias & 0.02 \\
\hline group & covariance & Intercept, Normative Accuracy & 0.00 \\
\hline group & covariance & Intercept, Distinctive Accuracy & 0.00 \\
\hline group & covariance & Intercept, P1 Extraversion & 0.00 \\
\hline group & covariance & Intercept, Normative Accuracy X P1 Extraversion & 0.00 \\
\hline group & covariance & Intercept, Distinctive Accuracy X P1 Extraversion & 0.00 \\
\hline group & covariance & Intercept, P1 Extraversion X Positivity Bias & 0.00 \\
\hline
\end{tabular}


NETWORK APPROACH TO REPUTATIONS: SUPPLEMENT

\begin{tabular}{|c|c|c|c|}
\hline source & type & term & Est. \\
\hline group & covariance & Normative Accuracy, Distinctive Accuracy & 0.00 \\
\hline group & covariance & Normative Accuracy, P1 Extraversion & 0.00 \\
\hline group & covariance & $\begin{array}{l}\text { Normative Accuracy, Normative Accuracy X P1 } \\
\text { Extraversion }\end{array}$ & -0.01 \\
\hline group & covariance & $\begin{array}{l}\text { Normative Accuracy, Distinctive Accuracy X P1 } \\
\text { Extraversion }\end{array}$ & 0.00 \\
\hline group & covariance & Normative Accuracy, P1 Extraversion X Positivity Bias & 0.02 \\
\hline group & covariance & Distinctive Accuracy, P1 Extraversion & 0.00 \\
\hline group & covariance & $\begin{array}{l}\text { Distinctive Accuracy, Normative Accuracy X P1 } \\
\text { Extraversion }\end{array}$ & 0.00 \\
\hline group & covariance & $\begin{array}{l}\text { Distinctive Accuracy, Distinctive Accuracy X P1 } \\
\text { Extraversion }\end{array}$ & 0.00 \\
\hline group & covariance & Distinctive Accuracy, P1 Extraversion X Positivity Bias & 0.00 \\
\hline group & covariance & P1 Extraversion, Normative Accuracy X P1 Extraversion & 0.00 \\
\hline group & covariance & P1 Extraversion, Distinctive Accuracy X P1 Extraversion & 0.00 \\
\hline group & covariance & P1 Extraversion, P1 Extraversion X Positivity Bias & 0.00 \\
\hline group & covariance & $\begin{array}{l}\text { Normative Accuracy X P1 Extraversion, Distinctive } \\
\text { Accuracy X P1 Extraversion }\end{array}$ & 0.00 \\
\hline group & covariance & $\begin{array}{l}\text { Normative Accuracy X P1 Extraversion, P1 Extraversion } \\
\text { X Positivity Bias }\end{array}$ & -0.01 \\
\hline group & covariance & $\begin{array}{l}\text { Distinctive Accuracy X P1 Extraversion, P1 Extraversion } \\
\text { X Positivity Bias }\end{array}$ & 0.00 \\
\hline Residual & & & 0.76 \\
\hline
\end{tabular}


NETWORK APPROACH TO REPUTATIONS: SUPPLEMENT

Table S26

Random Effects for P2 Extraversion Moderating Hearsay Accuracy (SAM)

\begin{tabular}{|c|c|c|c|}
\hline source & type & term & Est. \\
\hline triad:group & variance & Intercept & 0.00 \\
\hline triad:group & variance & Normative Accuracy & 0.03 \\
\hline triad:group & variance & Distinctive Accuracy & 0.00 \\
\hline triad:group & variance & P2 Extraversion & 0.01 \\
\hline triad:group & variance & Normative Accuracy X P2 Extraversion & 0.01 \\
\hline triad:group & variance & Distinctive Accuracy X P2 Extraversion & 0.01 \\
\hline triad:group & variance & P2 Extraversion X Positivity Bias & 0.13 \\
\hline triad:group & covariance & Intercept, Normative Accuracy & 0.00 \\
\hline triad:group & covariance & Intercept, Distinctive Accuracy & 0.00 \\
\hline triad:group & covariance & Intercept, P2 Extraversion & 0.00 \\
\hline triad:group & covariance & Intercept, Normative Accuracy X P2 Extraversion & 0.00 \\
\hline triad:group & covariance & Intercept, Distinctive Accuracy X P2 Extraversion & 0.00 \\
\hline triad:group & covariance & Intercept, P2 Extraversion X Positivity Bias & 0.00 \\
\hline triad:group & covariance & Normative Accuracy, Distinctive Accuracy & 0.00 \\
\hline triad:group & covariance & Normative Accuracy, P2 Extraversion & 0.00 \\
\hline triad:group & covariance & $\begin{array}{l}\text { Normative Accuracy, Normative Accuracy X P2 } \\
\text { Extraversion }\end{array}$ & 0.00 \\
\hline triad:group & covariance & $\begin{array}{l}\text { Normative Accuracy, Distinctive Accuracy X P2 } \\
\text { Extraversion }\end{array}$ & 0.00 \\
\hline triad:group & covariance & Normative Accuracy, P2 Extraversion X Positivity Bias & 0.01 \\
\hline triad:group & covariance & Distinctive Accuracy, P2 Extraversion & 0.00 \\
\hline triad:group & covariance & $\begin{array}{l}\text { Distinctive Accuracy, Normative Accuracy X P2 } \\
\text { Extraversion }\end{array}$ & 0.00 \\
\hline
\end{tabular}




\begin{tabular}{|c|c|c|c|}
\hline source & type & term & Est. \\
\hline triad:group & covariance & $\begin{array}{l}\text { Distinctive Accuracy, Distinctive Accuracy X P2 } \\
\text { Extraversion }\end{array}$ & 0.00 \\
\hline triad:group & covariance & Distinctive Accuracy, P2 Extraversion X Positivity Bias & 0.00 \\
\hline triad:group & covariance & P2 Extraversion, Normative Accuracy X P2 Extraversion & 0.00 \\
\hline triad:group & covariance & P2 Extraversion, Distinctive Accuracy X P2 Extraversion & -0.01 \\
\hline triad:group & covariance & P2 Extraversion, P2 Extraversion X Positivity Bias & -0.02 \\
\hline triad:group & covariance & $\begin{array}{l}\text { Normative Accuracy X P2 Extraversion, Distinctive } \\
\text { Accuracy X P2 Extraversion }\end{array}$ & 0.01 \\
\hline triad:group & covariance & $\begin{array}{l}\text { Normative Accuracy X P2 Extraversion, P2 Extraversion } \\
\text { X Positivity Bias }\end{array}$ & -0.01 \\
\hline triad:group & covariance & $\begin{array}{l}\text { Distinctive Accuracy X P2 Extraversion, P2 Extraversion } \\
\text { X Positivity Bias }\end{array}$ & 0.01 \\
\hline group & variance & Intercept & 0.00 \\
\hline group & variance & Normative Accuracy & 0.03 \\
\hline group & variance & Distinctive Accuracy & 0.00 \\
\hline group & variance & P2 Extraversion & 0.00 \\
\hline group & variance & Normative Accuracy X P2 Extraversion & 0.00 \\
\hline group & variance & Distinctive Accuracy X P2 Extraversion & 0.00 \\
\hline group & variance & P2 Extraversion X Positivity Bias & 0.04 \\
\hline group & covariance & Intercept, Normative Accuracy & 0.00 \\
\hline group & covariance & Intercept, Distinctive Accuracy & 0.00 \\
\hline group & covariance & Intercept, P2 Extraversion & 0.00 \\
\hline group & covariance & Intercept, Normative Accuracy X P2 Extraversion & 0.00 \\
\hline group & covariance & Intercept, Distinctive Accuracy X P2 Extraversion & 0.00 \\
\hline group & covariance & Intercept, P2 Extraversion X Positivity Bias & 0.00 \\
\hline
\end{tabular}


NETWORK APPROACH TO REPUTATIONS: SUPPLEMENT

\begin{tabular}{|c|c|c|c|}
\hline source & type & term & Est. \\
\hline group & covariance & Normative Accuracy, Distinctive Accuracy & 0.00 \\
\hline group & covariance & Normative Accuracy, P2 Extraversion & 0.00 \\
\hline group & covariance & $\begin{array}{l}\text { Normative Accuracy, Normative Accuracy X P2 } \\
\text { Extraversion }\end{array}$ & -0.01 \\
\hline group & covariance & $\begin{array}{l}\text { Normative Accuracy, Distinctive Accuracy X P2 } \\
\text { Extraversion }\end{array}$ & 0.00 \\
\hline group & covariance & Normative Accuracy, P2 Extraversion X Positivity Bias & 0.01 \\
\hline group & covariance & Distinctive Accuracy, P2 Extraversion & 0.00 \\
\hline group & covariance & $\begin{array}{l}\text { Distinctive Accuracy, Normative Accuracy X P2 } \\
\text { Extraversion }\end{array}$ & 0.00 \\
\hline group & covariance & $\begin{array}{l}\text { Distinctive Accuracy, Distinctive Accuracy X P2 } \\
\text { Extraversion }\end{array}$ & 0.00 \\
\hline group & covariance & Distinctive Accuracy, P2 Extraversion X Positivity Bias & 0.00 \\
\hline group & covariance & P2 Extraversion, Normative Accuracy X P2 Extraversion & 0.00 \\
\hline group & covariance & P2 Extraversion, Distinctive Accuracy X P2 Extraversion & 0.00 \\
\hline group & covariance & P2 Extraversion, P2 Extraversion X Positivity Bias & 0.00 \\
\hline group & covariance & $\begin{array}{l}\text { Normative Accuracy X P2 Extraversion, Distinctive } \\
\text { Accuracy X P2 Extraversion }\end{array}$ & 0.00 \\
\hline group & covariance & $\begin{array}{l}\text { Normative Accuracy X P2 Extraversion, P2 Extraversion } \\
\text { X Positivity Bias }\end{array}$ & 0.00 \\
\hline group & covariance & $\begin{array}{l}\text { Distinctive Accuracy X P2 Extraversion, P2 Extraversion } \\
\text { X Positivity Bias }\end{array}$ & 0.00 \\
\hline Residual & & & 0.76 \\
\hline
\end{tabular}


NETWORK APPROACH TO REPUTATIONS: SUPPLEMENT

Table S27

Random Effects for P2 Distress Moderating Hearsay Accuracy (SAM)

\begin{tabular}{|c|c|c|c|}
\hline source & type & term & Est. \\
\hline triad:group & variance & Intercept & 0.00 \\
\hline triad:group & variance & Normative Accuracy & 0.06 \\
\hline triad:group & variance & Distinctive Accuracy & 0.01 \\
\hline triad:group & variance & P2 Distress & 0.00 \\
\hline triad:group & variance & Normative Accuracy X P2 Distress & 0.01 \\
\hline triad:group & variance & Distinctive Accuracy X P2 Distress & 0.00 \\
\hline triad:group & variance & P2 Distress X Positivity Bias & 0.29 \\
\hline triad:group & covariance & Intercept, Normative Accuracy & -0.01 \\
\hline triad:group & covariance & Intercept, Distinctive Accuracy & 0.00 \\
\hline triad:group & covariance & Intercept, P2 Distress & 0.00 \\
\hline triad:group & covariance & Intercept, Normative Accuracy X P2 Distress & 0.00 \\
\hline triad:group & covariance & Intercept, Distinctive Accuracy X P2 Distress & 0.00 \\
\hline triad:group & covariance & Intercept, P2 Distress X Positivity Bias & 0.01 \\
\hline triad:group & covariance & Normative Accuracy, Distinctive Accuracy & 0.01 \\
\hline triad:group & covariance & Normative Accuracy, P2 Distress & 0.00 \\
\hline triad:group & covariance & Normative Accuracy, Normative Accuracy X P2 Distress & 0.00 \\
\hline triad:group & covariance & Normative Accuracy, Distinctive Accuracy X P2 Distress & 0.00 \\
\hline triad:group & covariance & Normative Accuracy, P2 Distress X Positivity Bias & -0.02 \\
\hline triad:group & covariance & Distinctive Accuracy, P2 Distress & 0.00 \\
\hline triad:group & covariance & Distinctive Accuracy, Normative Accuracy X P2 Distress & 0.00 \\
\hline triad:group & covariance & Distinctive Accuracy, Distinctive Accuracy X P2 Distress & 0.00 \\
\hline triad:group & covariance & Distinctive Accuracy, P2 Distress X Positivity Bias & -0.01 \\
\hline
\end{tabular}




\begin{tabular}{|c|c|c|c|}
\hline source & type & term & Est. \\
\hline triad:group & covariance & P2 Distress, Normative Accuracy X P2 Distress & 0.01 \\
\hline triad:group & covariance & P2 Distress, Distinctive Accuracy X P2 Distress & 0.00 \\
\hline triad:group & covariance & P2 Distress, P2 Distress X Positivity Bias & -0.03 \\
\hline triad:group & covariance & $\begin{array}{l}\text { Normative Accuracy X P2 Distress, Distinctive Accuracy } \\
\text { X P2 Distress }\end{array}$ & 0.00 \\
\hline triad:group & covariance & $\begin{array}{l}\text { Normative Accuracy X P2 Distress, P2 Distress X } \\
\text { Positivity Bias }\end{array}$ & -0.06 \\
\hline triad:group & covariance & $\begin{array}{l}\text { Distinctive Accuracy X P2 Distress, P2 Distress X } \\
\text { Positivity Bias }\end{array}$ & -0.01 \\
\hline group & variance & Intercept & 0.00 \\
\hline group & variance & Normative Accuracy & 0.01 \\
\hline group & variance & Distinctive Accuracy & 0.00 \\
\hline group & variance & P2 Distress & 0.00 \\
\hline group & variance & Normative Accuracy X P2 Distress & 0.01 \\
\hline group & variance & Distinctive Accuracy X P2 Distress & 0.00 \\
\hline group & variance & P2 Distress X Positivity Bias & 0.13 \\
\hline group & covariance & Intercept, Normative Accuracy & 0.00 \\
\hline group & covariance & Intercept, Distinctive Accuracy & 0.00 \\
\hline group & covariance & Intercept, P2 Distress & 0.00 \\
\hline group & covariance & Intercept, Normative Accuracy X P2 Distress & 0.00 \\
\hline group & covariance & Intercept, Distinctive Accuracy X P2 Distress & 0.00 \\
\hline group & covariance & Intercept, P2 Distress X Positivity Bias & 0.01 \\
\hline group & covariance & Normative Accuracy, Distinctive Accuracy & 0.00 \\
\hline group & covariance & Normative Accuracy, P2 Distress & 0.00 \\
\hline group & covariance & Normative Accuracy, Normative Accuracy X P2 Distress & -0.01 \\
\hline
\end{tabular}


NETWORK APPROACH TO REPUTATIONS: SUPPLEMENT

\begin{tabular}{|c|c|c|c|}
\hline source & type & term & Est. \\
\hline group & covariance & Normative Accuracy, Distinctive Accuracy X P2 Distress & 0.00 \\
\hline group & covariance & Normative Accuracy, P2 Distress X Positivity Bias & 0.02 \\
\hline group & covariance & Distinctive Accuracy, P2 Distress & 0.00 \\
\hline group & covariance & Distinctive Accuracy, Normative Accuracy X P2 Distress & 0.00 \\
\hline group & covariance & Distinctive Accuracy, Distinctive Accuracy X P2 Distress & 0.00 \\
\hline group & covariance & Distinctive Accuracy, P2 Distress X Positivity Bias & 0.00 \\
\hline group & covariance & P2 Distress, Normative Accuracy X P2 Distress & 0.00 \\
\hline group & covariance & P2 Distress, Distinctive Accuracy X P2 Distress & 0.00 \\
\hline group & covariance & P2 Distress, P2 Distress X Positivity Bias & -0.01 \\
\hline group & covariance & $\begin{array}{l}\text { Normative Accuracy X P2 Distress, Distinctive Accuracy } \\
\text { X P2 Distress }\end{array}$ & 0.00 \\
\hline group & covariance & $\begin{array}{l}\text { Normative Accuracy X P2 Distress, P2 Distress X } \\
\text { Positivity Bias }\end{array}$ & -0.04 \\
\hline group & covariance & $\begin{array}{l}\text { Distinctive Accuracy X P2 Distress, P2 Distress X } \\
\text { Positivity Bias }\end{array}$ & -0.01 \\
\hline Residual & & & 0.76 \\
\hline
\end{tabular}


NETWORK APPROACH TO REPUTATIONS: SUPPLEMENT

Table S28

Random Effects for P2 Empathic Concern Moderating Hearsay Accuracy (SAM)

\begin{tabular}{|c|c|c|c|}
\hline source & type & term & Est. \\
\hline triad:group & variance & Intercept & 0.00 \\
\hline triad:group & variance & Normative Accuracy & 0.04 \\
\hline triad:group & variance & Distinctive Accuracy & 0.01 \\
\hline triad:group & variance & P2 Empathic Concern & 0.01 \\
\hline triad:group & variance & Normative Accuracy X P2 Empathic Concern & 0.04 \\
\hline triad:group & variance & Distinctive Accuracy X P2 Empathic Concern & 0.00 \\
\hline triad:group & variance & P2 Empathic Concern X Positivity Bias & 0.83 \\
\hline triad:group & covariance & Intercept, Normative Accuracy & 0.00 \\
\hline triad:group & covariance & Intercept, Distinctive Accuracy & 0.00 \\
\hline triad:group & covariance & Intercept, P2 Empathic Concern & 0.00 \\
\hline triad:group & covariance & Intercept, Normative Accuracy X P2 Empathic Concern & 0.00 \\
\hline triad:group & covariance & Intercept, Distinctive Accuracy X P2 Empathic Concern & 0.00 \\
\hline triad:group & covariance & Intercept, P2 Empathic Concern X Positivity Bias & 0.00 \\
\hline triad:group & covariance & Normative Accuracy, Distinctive Accuracy & 0.01 \\
\hline triad:group & covariance & Normative Accuracy, P2 Empathic Concern & 0.00 \\
\hline triad:group & covariance & $\begin{array}{l}\text { Normative Accuracy, Normative Accuracy X P2 Empathic } \\
\text { Concern }\end{array}$ & 0.01 \\
\hline triad:group & covariance & $\begin{array}{l}\text { Normative Accuracy, Distinctive Accuracy X P2 Empathic } \\
\text { Concern }\end{array}$ & 0.00 \\
\hline triad:group & covariance & $\begin{array}{l}\text { Normative Accuracy, P2 Empathic Concern X Positivity } \\
\text { Bias }\end{array}$ & -0.02 \\
\hline triad:group & covariance & Distinctive Accuracy, P2 Empathic Concern & 0.00 \\
\hline triad:group & covariance & $\begin{array}{l}\text { Distinctive Accuracy, Normative Accuracy X P2 Empathic } \\
\text { Concern }\end{array}$ & 0.01 \\
\hline
\end{tabular}




\begin{tabular}{|c|c|c|c|}
\hline source & type & term & Est. \\
\hline triad:group & covariance & $\begin{array}{l}\text { Distinctive Accuracy, Distinctive Accuracy X P2 Empathic } \\
\text { Concern }\end{array}$ & 0.01 \\
\hline triad:group & covariance & $\begin{array}{l}\text { Distinctive Accuracy, P2 Empathic Concern X Positivity } \\
\text { Bias }\end{array}$ & -0.01 \\
\hline triad:group & covariance & $\begin{array}{l}\text { P2 Empathic Concern, Normative Accuracy X P2 } \\
\text { Empathic Concern }\end{array}$ & 0.01 \\
\hline triad:group & covariance & $\begin{array}{l}\text { P2 Empathic Concern, Distinctive Accuracy X P2 } \\
\text { Empathic Concern }\end{array}$ & 0.00 \\
\hline triad:group & covariance & $\begin{array}{l}\text { P2 Empathic Concern, P2 Empathic Concern X Positivity } \\
\text { Bias }\end{array}$ & -0.08 \\
\hline triad:group & covariance & $\begin{array}{l}\text { Normative Accuracy X P2 Empathic Concern, Distinctive } \\
\text { Accuracy X P2 Empathic Concern }\end{array}$ & 0.01 \\
\hline triad:group & covariance & $\begin{array}{l}\text { Normative Accuracy X P2 Empathic Concern, P2 } \\
\text { Empathic Concern X Positivity Bias }\end{array}$ & -0.16 \\
\hline triad:group & covariance & $\begin{array}{l}\text { Distinctive Accuracy X P2 Empathic Concern, P2 } \\
\text { Empathic Concern X Positivity Bias }\end{array}$ & -0.01 \\
\hline group & variance & Intercept & 0.00 \\
\hline group & variance & Normative Accuracy & 0.00 \\
\hline group & variance & Distinctive Accuracy & 0.00 \\
\hline group & variance & P2 Empathic Concern & 0.00 \\
\hline group & variance & Normative Accuracy X P2 Empathic Concern & 0.01 \\
\hline group & variance & Distinctive Accuracy X P2 Empathic Concern & 0.00 \\
\hline group & variance & P2 Empathic Concern X Positivity Bias & 0.04 \\
\hline group & covariance & Intercept, Normative Accuracy & 0.00 \\
\hline group & covariance & Intercept, Distinctive Accuracy & 0.00 \\
\hline group & covariance & Intercept, P2 Empathic Concern & 0.00 \\
\hline group & covariance & Intercept, Normative Accuracy X P2 Empathic Concern & 0.01 \\
\hline
\end{tabular}




\begin{tabular}{|c|c|c|c|}
\hline source & type & term & Est. \\
\hline group & covariance & Intercept, Distinctive Accuracy X P2 Empathic Concern & 0.00 \\
\hline group & covariance & Intercept, P2 Empathic Concern X Positivity Bias & 0.00 \\
\hline group & covariance & Normative Accuracy, Distinctive Accuracy & 0.00 \\
\hline group & covariance & Normative Accuracy, P2 Empathic Concern & 0.00 \\
\hline group & covariance & $\begin{array}{l}\text { Normative Accuracy, Normative Accuracy X P2 Empathic } \\
\text { Concern }\end{array}$ & 0.00 \\
\hline group & covariance & $\begin{array}{l}\text { Normative Accuracy, Distinctive Accuracy X P2 Empathic } \\
\text { Concern }\end{array}$ & 0.00 \\
\hline group & covariance & $\begin{array}{l}\text { Normative Accuracy, P2 Empathic Concern X Positivity } \\
\text { Bias }\end{array}$ & 0.00 \\
\hline group & covariance & Distinctive Accuracy, P2 Empathic Concern & 0.00 \\
\hline group & covariance & $\begin{array}{l}\text { Distinctive Accuracy, Normative Accuracy X P2 Empathic } \\
\text { Concern }\end{array}$ & 0.00 \\
\hline group & covariance & $\begin{array}{l}\text { Distinctive Accuracy, Distinctive Accuracy X P2 Empathic } \\
\text { Concern }\end{array}$ & 0.00 \\
\hline group & covariance & $\begin{array}{l}\text { Distinctive Accuracy, P2 Empathic Concern X Positivity } \\
\text { Bias }\end{array}$ & -0.01 \\
\hline group & covariance & $\begin{array}{l}\text { P2 Empathic Concern, Normative Accuracy X P2 } \\
\text { Empathic Concern }\end{array}$ & 0.00 \\
\hline group & covariance & $\begin{array}{l}\text { P2 Empathic Concern, Distinctive Accuracy X P2 } \\
\text { Empathic Concern }\end{array}$ & 0.00 \\
\hline group & covariance & $\begin{array}{l}\text { P2 Empathic Concern, P2 Empathic Concern X Positivity } \\
\text { Bias }\end{array}$ & 0.00 \\
\hline group & covariance & $\begin{array}{l}\text { Normative Accuracy X P2 Empathic Concern, Distinctive } \\
\text { Accuracy X P2 Empathic Concern }\end{array}$ & 0.00 \\
\hline group & covariance & $\begin{array}{l}\text { Normative Accuracy X P2 Empathic Concern, P2 } \\
\text { Empathic Concern X Positivity Bias }\end{array}$ & 0.00 \\
\hline group & covariance & $\begin{array}{l}\text { Distinctive Accuracy X P2 Empathic Concern, P2 } \\
\text { Empathic Concern X Positivity Bias }\end{array}$ & 0.00 \\
\hline
\end{tabular}


NETWORK APPROACH TO REPUTATIONS: SUPPLEMENT

\begin{tabular}{llrr}
\hline source & type & term & Est. \\
\hline Residual & & 0.75 \\
\hline
\end{tabular}


NETWORK APPROACH TO REPUTATIONS: SUPPLEMENT

Table S29

Random Effects for P2 Distress Moderating Hearsay Consensus (SAM)

\begin{tabular}{|c|c|c|c|}
\hline source & type & term & Est. \\
\hline triad:group & variance & Intercept & 0.00 \\
\hline triad:group & variance & Normative Consensus & 0.14 \\
\hline triad:group & variance & Distinctive Consensus & 0.02 \\
\hline triad:group & variance & P2 Distress & 0.00 \\
\hline triad:group & variance & Normative Consensus X P2 Distress & 0.09 \\
\hline triad:group & variance & Distinctive Consensus X P2 Distress & 0.01 \\
\hline triad:group & variance & P2 Distress X Positivity Bias & 0.18 \\
\hline triad:group & covariance & Intercept, Normative Consensus & -0.02 \\
\hline triad:group & covariance & Intercept, Distinctive Consensus & 0.00 \\
\hline triad:group & covariance & Intercept, P2 Distress & 0.00 \\
\hline triad:group & covariance & Intercept, Normative Consensus X P2 Distress & 0.00 \\
\hline triad:group & covariance & Intercept, Distinctive Consensus X P2 Distress & 0.00 \\
\hline triad:group & covariance & Intercept, P2 Distress X Positivity Bias & 0.00 \\
\hline triad:group & covariance & Normative Consensus, Distinctive Consensus & 0.00 \\
\hline triad:group & covariance & Normative Consensus, P2 Distress & 0.00 \\
\hline triad:group & covariance & $\begin{array}{l}\text { Normative Consensus, Normative Consensus X P2 } \\
\text { Distress }\end{array}$ & -0.04 \\
\hline triad:group & covariance & $\begin{array}{l}\text { Normative Consensus, Distinctive Consensus X P2 } \\
\text { Distress }\end{array}$ & 0.00 \\
\hline triad:group & covariance & Normative Consensus, P2 Distress X Positivity Bias & 0.04 \\
\hline triad:group & covariance & Distinctive Consensus, P2 Distress & 0.01 \\
\hline triad:group & covariance & $\begin{array}{l}\text { Distinctive Consensus, Normative Consensus X P2 } \\
\text { Distress }\end{array}$ & 0.01 \\
\hline
\end{tabular}


NETWORK APPROACH TO REPUTATIONS: SUPPLEMENT

\begin{tabular}{|c|c|c|c|}
\hline source & type & term & Est. \\
\hline triad:group & covariance & $\begin{array}{l}\text { Distinctive Consensus, Distinctive Consensus X P2 } \\
\text { Distress }\end{array}$ & 0.01 \\
\hline triad:group & covariance & Distinctive Consensus, P2 Distress X Positivity Bias & -0.02 \\
\hline triad:group & covariance & P2 Distress, Normative Consensus X P2 Distress & 0.00 \\
\hline triad:group & covariance & P2 Distress, Distinctive Consensus X P2 Distress & 0.00 \\
\hline triad:group & covariance & P2 Distress, P2 Distress X Positivity Bias & -0.01 \\
\hline triad:group & covariance & $\begin{array}{l}\text { Normative Consensus X P2 Distress, Distinctive } \\
\text { Consensus X P2 Distress }\end{array}$ & 0.00 \\
\hline triad:group & covariance & $\begin{array}{l}\text { Normative Consensus X P2 Distress, P2 Distress X } \\
\text { Positivity Bias }\end{array}$ & -0.12 \\
\hline triad:group & covariance & $\begin{array}{l}\text { Distinctive Consensus X P2 Distress, P2 Distress X } \\
\text { Positivity Bias }\end{array}$ & -0.01 \\
\hline group & variance & Intercept & 0.00 \\
\hline group & variance & Normative Consensus & 0.02 \\
\hline group & variance & Distinctive Consensus & 0.01 \\
\hline group & variance & P2 Distress & 0.00 \\
\hline group & variance & Normative Consensus X P2 Distress & 0.08 \\
\hline group & variance & Distinctive Consensus X P2 Distress & 0.00 \\
\hline group & variance & P2 Distress X Positivity Bias & 0.09 \\
\hline group & covariance & Intercept, Normative Consensus & 0.00 \\
\hline group & covariance & Intercept, Distinctive Consensus & 0.00 \\
\hline group & covariance & Intercept, P2 Distress & 0.00 \\
\hline group & covariance & Intercept, Normative Consensus X P2 Distress & 0.00 \\
\hline group & covariance & Intercept, Distinctive Consensus X P2 Distress & 0.00 \\
\hline group & covariance & Intercept, P2 Distress X Positivity Bias & 0.01 \\
\hline
\end{tabular}


NETWORK APPROACH TO REPUTATIONS: SUPPLEMENT

\begin{tabular}{|c|c|c|c|}
\hline source & type & term & Est. \\
\hline group & covariance & Normative Consensus, Distinctive Consensus & 0.00 \\
\hline group & covariance & Normative Consensus, P2 Distress & 0.00 \\
\hline group & covariance & $\begin{array}{l}\text { Normative Consensus, Normative Consensus X P2 } \\
\text { Distress }\end{array}$ & 0.03 \\
\hline group & covariance & $\begin{array}{l}\text { Normative Consensus, Distinctive Consensus X P2 } \\
\text { Distress }\end{array}$ & 0.00 \\
\hline group & covariance & Normative Consensus, P2 Distress X Positivity Bias & -0.02 \\
\hline group & covariance & Distinctive Consensus, P2 Distress & 0.00 \\
\hline group & covariance & $\begin{array}{l}\text { Distinctive Consensus, Normative Consensus X P2 } \\
\text { Distress }\end{array}$ & 0.01 \\
\hline group & covariance & $\begin{array}{l}\text { Distinctive Consensus, Distinctive Consensus X P2 } \\
\text { Distress }\end{array}$ & 0.00 \\
\hline group & covariance & Distinctive Consensus, P2 Distress X Positivity Bias & -0.01 \\
\hline group & covariance & P2 Distress, Normative Consensus X P2 Distress & 0.00 \\
\hline group & covariance & P2 Distress, Distinctive Consensus X P2 Distress & 0.00 \\
\hline group & covariance & P2 Distress, P2 Distress X Positivity Bias & 0.00 \\
\hline group & covariance & $\begin{array}{l}\text { Normative Consensus X P2 Distress, Distinctive } \\
\text { Consensus X P2 Distress }\end{array}$ & 0.01 \\
\hline group & covariance & $\begin{array}{l}\text { Normative Consensus X P2 Distress, P2 Distress X } \\
\text { Positivity Bias }\end{array}$ & -0.08 \\
\hline group & covariance & $\begin{array}{l}\text { Distinctive Consensus X P2 Distress, P2 Distress X } \\
\text { Positivity Bias }\end{array}$ & 0.00 \\
\hline Residual & & & 0.69 \\
\hline
\end{tabular}


NETWORK APPROACH TO REPUTATIONS: SUPPLEMENT

Table S30

Random Effects for P2 Empathic Concern Moderating Hearsay Consensus (SAM)

\begin{tabular}{|c|c|c|c|}
\hline source & type & term & Est. \\
\hline triad:group & variance & Intercept & 0.00 \\
\hline triad:group & variance & Normative Consensus & 0.12 \\
\hline triad:group & variance & Distinctive Consensus & 0.02 \\
\hline triad:group & variance & P2 Empathic Concern & 0.00 \\
\hline triad:group & variance & Normative Consensus X P2 Empathic Concern & 0.26 \\
\hline triad:group & variance & Distinctive Consensus X P2 Empathic Concern & 0.00 \\
\hline triad:group & variance & P2 Empathic Concern X Positivity Bias & 0.45 \\
\hline triad:group & covariance & Intercept, Normative Consensus & -0.01 \\
\hline triad:group & covariance & Intercept, Distinctive Consensus & 0.00 \\
\hline triad:group & covariance & Intercept, P2 Empathic Concern & 0.00 \\
\hline triad:group & covariance & Intercept, Normative Consensus X P2 Empathic Concern & 0.01 \\
\hline triad:group & covariance & Intercept, Distinctive Consensus X P2 Empathic Concern & 0.00 \\
\hline triad:group & covariance & Intercept, P2 Empathic Concern X Positivity Bias & -0.01 \\
\hline triad:group & covariance & Normative Consensus, Distinctive Consensus & 0.00 \\
\hline triad:group & covariance & Normative Consensus, P2 Empathic Concern & -0.01 \\
\hline triad:group & covariance & $\begin{array}{l}\text { Normative Consensus, Normative Consensus X P2 } \\
\text { Empathic Concern }\end{array}$ & -0.02 \\
\hline triad:group & covariance & $\begin{array}{l}\text { Normative Consensus, Distinctive Consensus X P2 } \\
\text { Empathic Concern }\end{array}$ & 0.00 \\
\hline triad:group & covariance & $\begin{array}{l}\text { Normative Consensus, P2 Empathic Concern X Positivity } \\
\text { Bias }\end{array}$ & 0.05 \\
\hline triad:group & covariance & Distinctive Consensus, P2 Empathic Concern & 0.00 \\
\hline triad:group & covariance & $\begin{array}{l}\text { Distinctive Consensus, Normative Consensus X P2 } \\
\text { Empathic Concern }\end{array}$ & 0.02 \\
\hline
\end{tabular}




\begin{tabular}{|c|c|c|c|}
\hline source & type & term & Est. \\
\hline triad:group & covariance & $\begin{array}{l}\text { Distinctive Consensus, Distinctive Consensus X P2 } \\
\text { Empathic Concern }\end{array}$ & 0.00 \\
\hline triad:group & covariance & $\begin{array}{l}\text { Distinctive Consensus, P2 Empathic Concern X Positivity } \\
\text { Bias }\end{array}$ & -0.04 \\
\hline triad:group & covariance & $\begin{array}{l}\text { P2 Empathic Concern, Normative Consensus X P2 } \\
\text { Empathic Concern }\end{array}$ & -0.01 \\
\hline triad:group & covariance & $\begin{array}{l}\text { P2 Empathic Concern, Distinctive Consensus X P2 } \\
\text { Empathic Concern }\end{array}$ & 0.00 \\
\hline triad:group & covariance & $\begin{array}{l}\text { P2 Empathic Concern, P2 Empathic Concern X Positivity } \\
\text { Bias }\end{array}$ & 0.01 \\
\hline triad:group & covariance & $\begin{array}{l}\text { Normative Consensus X P2 Empathic Concern, } \\
\text { Distinctive Consensus X P2 Empathic Concern }\end{array}$ & 0.01 \\
\hline triad:group & covariance & $\begin{array}{l}\text { Normative Consensus X P2 Empathic Concern, P2 } \\
\text { Empathic Concern X Positivity Bias }\end{array}$ & -0.34 \\
\hline triad:group & covariance & $\begin{array}{l}\text { Distinctive Consensus X P2 Empathic Concern, P2 } \\
\text { Empathic Concern X Positivity Bias }\end{array}$ & 0.00 \\
\hline group & variance & Intercept & 0.00 \\
\hline group & variance & Normative Consensus & 0.01 \\
\hline group & variance & Distinctive Consensus & 0.01 \\
\hline group & variance & P2 Empathic Concern & 0.00 \\
\hline group & variance & Normative Consensus X P2 Empathic Concern & 0.03 \\
\hline group & variance & Distinctive Consensus X P2 Empathic Concern & 0.00 \\
\hline group & variance & P2 Empathic Concern X Positivity Bias & 0.01 \\
\hline group & covariance & Intercept, Normative Consensus & 0.00 \\
\hline group & covariance & Intercept, Distinctive Consensus & 0.00 \\
\hline group & covariance & Intercept, P2 Empathic Concern & 0.00 \\
\hline group & covariance & Intercept, Normative Consensus X P2 Empathic Concern & 0.00 \\
\hline
\end{tabular}




\begin{tabular}{|c|c|c|c|}
\hline source & type & term & Est. \\
\hline group & covariance & Intercept, Distinctive Consensus X P2 Empathic Concern & 0.00 \\
\hline group & covariance & Intercept, P2 Empathic Concern X Positivity Bias & 0.00 \\
\hline group & covariance & Normative Consensus, Distinctive Consensus & 0.00 \\
\hline group & covariance & Normative Consensus, P2 Empathic Concern & 0.00 \\
\hline group & covariance & $\begin{array}{l}\text { Normative Consensus, Normative Consensus X P2 } \\
\text { Empathic Concern }\end{array}$ & 0.00 \\
\hline group & covariance & $\begin{array}{l}\text { Normative Consensus, Distinctive Consensus X P2 } \\
\text { Empathic Concern }\end{array}$ & 0.00 \\
\hline group & covariance & $\begin{array}{l}\text { Normative Consensus, P2 Empathic Concern X Positivity } \\
\text { Bias }\end{array}$ & -0.01 \\
\hline group & covariance & Distinctive Consensus, P2 Empathic Concern & 0.00 \\
\hline group & covariance & $\begin{array}{l}\text { Distinctive Consensus, Normative Consensus X P2 } \\
\text { Empathic Concern }\end{array}$ & 0.01 \\
\hline group & covariance & $\begin{array}{l}\text { Distinctive Consensus, Distinctive Consensus X P2 } \\
\text { Empathic Concern }\end{array}$ & 0.00 \\
\hline group & covariance & $\begin{array}{l}\text { Distinctive Consensus, P2 Empathic Concern X Positivity } \\
\text { Bias }\end{array}$ & 0.00 \\
\hline group & covariance & $\begin{array}{l}\text { P2 Empathic Concern, Normative Consensus X P2 } \\
\text { Empathic Concern }\end{array}$ & 0.00 \\
\hline group & covariance & $\begin{array}{l}\text { P2 Empathic Concern, Distinctive Consensus X P2 } \\
\text { Empathic Concern }\end{array}$ & 0.00 \\
\hline group & covariance & $\begin{array}{l}\text { P2 Empathic Concern, P2 Empathic Concern X Positivity } \\
\text { Bias }\end{array}$ & 0.00 \\
\hline group & covariance & $\begin{array}{l}\text { Normative Consensus X P2 Empathic Concern, } \\
\text { Distinctive Consensus X P2 Empathic Concern }\end{array}$ & 0.00 \\
\hline group & covariance & $\begin{array}{l}\text { Normative Consensus X P2 Empathic Concern, P2 } \\
\text { Empathic Concern X Positivity Bias }\end{array}$ & 0.00 \\
\hline group & covariance & $\begin{array}{l}\text { Distinctive Consensus X P2 Empathic Concern, P2 } \\
\text { Empathic Concern X Positivity Bias }\end{array}$ & 0.00 \\
\hline
\end{tabular}


NETWORK APPROACH TO REPUTATIONS: SUPPLEMENT

\begin{tabular}{llrr}
\hline source & type & term & Est. \\
\hline Residual & & 0.69 \\
\hline
\end{tabular}


NETWORK APPROACH TO REPUTATIONS: SUPPLEMENT

Table S31

Random Effects for P2 Fantasy Moderating Hearsay Consensus (SAM)

\begin{tabular}{|c|c|c|c|}
\hline source & type & term & Est. \\
\hline triad:group & variance & Intercept & 0.00 \\
\hline triad:group & variance & Normative Consensus & 0.12 \\
\hline triad:group & variance & Distinctive Consensus & 0.02 \\
\hline triad:group & variance & P2 Fantasy & 0.00 \\
\hline triad:group & variance & Normative Consensus X P2 Fantasy & 0.11 \\
\hline triad:group & variance & Distinctive Consensus X P2 Fantasy & 0.00 \\
\hline triad:group & variance & P2 Fantasy X Positivity Bias & 0.20 \\
\hline triad:group & covariance & Intercept, Normative Consensus & -0.02 \\
\hline triad:group & covariance & Intercept, Distinctive Consensus & 0.00 \\
\hline triad:group & covariance & Intercept, P2 Fantasy & 0.00 \\
\hline triad:group & covariance & Intercept, Normative Consensus X P2 Fantasy & 0.00 \\
\hline triad:group & covariance & Intercept, Distinctive Consensus X P2 Fantasy & 0.00 \\
\hline triad:group & covariance & Intercept, P2 Fantasy X Positivity Bias & 0.00 \\
\hline triad:group & covariance & Normative Consensus, Distinctive Consensus & 0.00 \\
\hline triad:group & covariance & Normative Consensus, P2 Fantasy & 0.00 \\
\hline triad:group & covariance & Normative Consensus, Normative Consensus X P2 Fantasy & -0.01 \\
\hline triad:group & covariance & $\begin{array}{l}\text { Normative Consensus, Distinctive Consensus X P2 } \\
\text { Fantasy }\end{array}$ & -0.02 \\
\hline triad:group & covariance & Normative Consensus, P2 Fantasy X Positivity Bias & 0.00 \\
\hline triad:group & covariance & Distinctive Consensus, P2 Fantasy & 0.00 \\
\hline triad:group & covariance & $\begin{array}{l}\text { Distinctive Consensus, Normative Consensus X P2 } \\
\text { Fantasy }\end{array}$ & 0.01 \\
\hline
\end{tabular}


NETWORK APPROACH TO REPUTATIONS: SUPPLEMENT

\begin{tabular}{|c|c|c|c|}
\hline source & type & term & Est. \\
\hline triad:group & covariance & $\begin{array}{l}\text { Distinctive Consensus, Distinctive Consensus X P2 } \\
\text { Fantasy }\end{array}$ & 0.00 \\
\hline triad:group & covariance & Distinctive Consensus, P2 Fantasy X Positivity Bias & -0.02 \\
\hline triad:group & covariance & P2 Fantasy, Normative Consensus X P2 Fantasy & 0.00 \\
\hline triad:group & covariance & P2 Fantasy, Distinctive Consensus X P2 Fantasy & 0.00 \\
\hline triad:group & covariance & P2 Fantasy, P2 Fantasy X Positivity Bias & 0.00 \\
\hline triad:group & covariance & $\begin{array}{l}\text { Normative Consensus X P2 Fantasy, Distinctive } \\
\text { Consensus X P2 Fantasy }\end{array}$ & 0.01 \\
\hline triad:group & covariance & $\begin{array}{l}\text { Normative Consensus X P2 Fantasy, P2 Fantasy X } \\
\text { Positivity Bias }\end{array}$ & -0.14 \\
\hline triad:group & covariance & $\begin{array}{l}\text { Distinctive Consensus X P2 Fantasy, P2 Fantasy X } \\
\text { Positivity Bias }\end{array}$ & -0.01 \\
\hline group & variance & Intercept & 0.00 \\
\hline group & variance & Normative Consensus & 0.02 \\
\hline group & variance & Distinctive Consensus & 0.01 \\
\hline group & variance & P2 Fantasy & 0.00 \\
\hline group & variance & Normative Consensus X P2 Fantasy & 0.00 \\
\hline group & variance & Distinctive Consensus X P2 Fantasy & 0.00 \\
\hline group & variance & P2 Fantasy X Positivity Bias & 0.01 \\
\hline group & covariance & Intercept, Normative Consensus & 0.00 \\
\hline group & covariance & Intercept, Distinctive Consensus & 0.00 \\
\hline group & covariance & Intercept, P2 Fantasy & 0.00 \\
\hline group & covariance & Intercept, Normative Consensus X P2 Fantasy & 0.00 \\
\hline group & covariance & Intercept, Distinctive Consensus X P2 Fantasy & 0.00 \\
\hline group & covariance & Intercept, P2 Fantasy X Positivity Bias & 0.00 \\
\hline
\end{tabular}


NETWORK APPROACH TO REPUTATIONS: SUPPLEMENT

\begin{tabular}{|c|c|c|c|}
\hline source & type & term & Est. \\
\hline group & covariance & Normative Consensus, Distinctive Consensus & 0.01 \\
\hline group & covariance & Normative Consensus, P2 Fantasy & 0.00 \\
\hline group & covariance & Normative Consensus, Normative Consensus X P2 Fantasy & 0.00 \\
\hline group & covariance & $\begin{array}{l}\text { Normative Consensus, Distinctive Consensus X P2 } \\
\text { Fantasy }\end{array}$ & 0.00 \\
\hline group & covariance & Normative Consensus, P2 Fantasy X Positivity Bias & 0.01 \\
\hline group & covariance & Distinctive Consensus, P2 Fantasy & 0.00 \\
\hline group & covariance & $\begin{array}{l}\text { Distinctive Consensus, Normative Consensus X P2 } \\
\text { Fantasy }\end{array}$ & 0.00 \\
\hline group & covariance & $\begin{array}{l}\text { Distinctive Consensus, Distinctive Consensus X P2 } \\
\text { Fantasy }\end{array}$ & 0.00 \\
\hline group & covariance & Distinctive Consensus, P2 Fantasy X Positivity Bias & 0.01 \\
\hline group & covariance & P2 Fantasy, Normative Consensus X P2 Fantasy & 0.00 \\
\hline group & covariance & P2 Fantasy, Distinctive Consensus X P2 Fantasy & 0.00 \\
\hline group & covariance & P2 Fantasy, P2 Fantasy X Positivity Bias & 0.00 \\
\hline group & covariance & $\begin{array}{l}\text { Normative Consensus X P2 Fantasy, Distinctive } \\
\text { Consensus X P2 Fantasy }\end{array}$ & 0.00 \\
\hline group & covariance & $\begin{array}{l}\text { Normative Consensus X P2 Fantasy, P2 Fantasy X } \\
\text { Positivity Bias }\end{array}$ & 0.00 \\
\hline group & covariance & $\begin{array}{l}\text { Distinctive Consensus X P2 Fantasy, P2 Fantasy X } \\
\text { Positivity Bias }\end{array}$ & 0.00 \\
\hline Residual & & & 0.69 \\
\hline
\end{tabular}


NETWORK APPROACH TO REPUTATIONS: SUPPLEMENT

Table S32

Random Effects for P2 Perspective Taking Moderating Hearsay Consensus (SAM)

\begin{tabular}{|c|c|c|c|}
\hline source & type & term & Est. \\
\hline triad:group & variance & Intercept & 0.00 \\
\hline triad:group & variance & Normative Consensus & 0.12 \\
\hline triad:group & variance & Distinctive Consensus & 0.02 \\
\hline triad:group & variance & P2 Perspective Taking & 0.00 \\
\hline triad:group & variance & Normative Consensus X P2 Perspective Taking & 0.19 \\
\hline triad:group & variance & Distinctive Consensus X P2 Perspective Taking & 0.01 \\
\hline triad:group & variance & P2 Perspective Taking X Positivity Bias & 0.34 \\
\hline triad:group & covariance & Intercept, Normative Consensus & -0.02 \\
\hline triad:group & covariance & Intercept, Distinctive Consensus & 0.00 \\
\hline triad:group & covariance & Intercept, P2 Perspective Taking & 0.00 \\
\hline triad:group & covariance & Intercept, Normative Consensus X P2 Perspective Taking & 0.00 \\
\hline triad:group & covariance & Intercept, Distinctive Consensus X P2 Perspective Taking & 0.00 \\
\hline triad:group & covariance & Intercept, P2 Perspective Taking X Positivity Bias & 0.00 \\
\hline triad:group & covariance & Normative Consensus, Distinctive Consensus & 0.00 \\
\hline triad:group & covariance & Normative Consensus, P2 Perspective Taking & 0.01 \\
\hline triad:group & covariance & $\begin{array}{l}\text { Normative Consensus, Normative Consensus X P2 } \\
\text { Perspective Taking }\end{array}$ & -0.02 \\
\hline triad:group & covariance & $\begin{array}{l}\text { Normative Consensus, Distinctive Consensus X P2 } \\
\text { Perspective Taking }\end{array}$ & 0.00 \\
\hline triad:group & covariance & $\begin{array}{l}\text { Normative Consensus, P2 Perspective Taking X Positivity } \\
\text { Bias }\end{array}$ & 0.02 \\
\hline triad:group & covariance & Distinctive Consensus, P2 Perspective Taking & 0.00 \\
\hline triad:group & covariance & $\begin{array}{l}\text { Distinctive Consensus, Normative Consensus X P2 } \\
\text { Perspective Taking }\end{array}$ & 0.02 \\
\hline
\end{tabular}




\begin{tabular}{|c|c|c|c|}
\hline source & type & term & Est. \\
\hline triad:group & covariance & $\begin{array}{l}\text { Distinctive Consensus, Distinctive Consensus X P2 } \\
\text { Perspective Taking }\end{array}$ & -0.01 \\
\hline triad:group & covariance & $\begin{array}{l}\text { Distinctive Consensus, P2 Perspective Taking X } \\
\text { Positivity Bias }\end{array}$ & -0.03 \\
\hline triad:group & covariance & $\begin{array}{l}\text { P2 Perspective Taking, Normative Consensus X P2 } \\
\text { Perspective Taking }\end{array}$ & 0.01 \\
\hline triad:group & covariance & $\begin{array}{l}\text { P2 Perspective Taking, Distinctive Consensus X P2 } \\
\text { Perspective Taking }\end{array}$ & 0.00 \\
\hline triad:group & covariance & $\begin{array}{l}\text { P2 Perspective Taking, P2 Perspective Taking X } \\
\text { Positivity Bias }\end{array}$ & -0.02 \\
\hline triad:group & covariance & $\begin{array}{l}\text { Normative Consensus X P2 Perspective Taking, } \\
\text { Distinctive Consensus X P2 Perspective Taking }\end{array}$ & 0.00 \\
\hline triad:group & covariance & $\begin{array}{l}\text { Normative Consensus X P2 Perspective Taking, P2 } \\
\text { Perspective Taking X Positivity Bias }\end{array}$ & -0.25 \\
\hline triad:group & covariance & $\begin{array}{l}\text { Distinctive Consensus X P2 Perspective Taking, P2 } \\
\text { Perspective Taking X Positivity Bias }\end{array}$ & 0.00 \\
\hline group & variance & Intercept & 0.00 \\
\hline group & variance & Normative Consensus & 0.04 \\
\hline group & variance & Distinctive Consensus & 0.00 \\
\hline group & variance & P2 Perspective Taking & 0.00 \\
\hline group & variance & Normative Consensus X P2 Perspective Taking & 0.01 \\
\hline group & variance & Distinctive Consensus X P2 Perspective Taking & 0.00 \\
\hline group & variance & P2 Perspective Taking X Positivity Bias & 0.01 \\
\hline group & covariance & Intercept, Normative Consensus & 0.00 \\
\hline group & covariance & Intercept, Distinctive Consensus & 0.00 \\
\hline group & covariance & Intercept, P2 Perspective Taking & 0.00 \\
\hline group & covariance & Intercept, Normative Consensus X P2 Perspective Taking & 0.00 \\
\hline
\end{tabular}




\begin{tabular}{|c|c|c|c|}
\hline source & type & term & Est. \\
\hline group & covariance & Intercept, Distinctive Consensus X P2 Perspective Taking & 0.00 \\
\hline group & covariance & Intercept, P2 Perspective Taking X Positivity Bias & 0.00 \\
\hline group & covariance & Normative Consensus, Distinctive Consensus & 0.00 \\
\hline group & covariance & Normative Consensus, P2 Perspective Taking & 0.00 \\
\hline group & covariance & $\begin{array}{l}\text { Normative Consensus, Normative Consensus X P2 } \\
\text { Perspective Taking }\end{array}$ & 0.01 \\
\hline group & covariance & $\begin{array}{l}\text { Normative Consensus, Distinctive Consensus X P2 } \\
\text { Perspective Taking }\end{array}$ & 0.00 \\
\hline group & covariance & $\begin{array}{l}\text { Normative Consensus, P2 Perspective Taking X Positivity } \\
\text { Bias }\end{array}$ & -0.01 \\
\hline group & covariance & Distinctive Consensus, P2 Perspective Taking & 0.00 \\
\hline group & covariance & $\begin{array}{l}\text { Distinctive Consensus, Normative Consensus X P2 } \\
\text { Perspective Taking }\end{array}$ & 0.00 \\
\hline group & covariance & $\begin{array}{l}\text { Distinctive Consensus, Distinctive Consensus X P2 } \\
\text { Perspective Taking }\end{array}$ & 0.00 \\
\hline group & covariance & $\begin{array}{l}\text { Distinctive Consensus, P2 Perspective Taking X } \\
\text { Positivity Bias }\end{array}$ & 0.00 \\
\hline group & covariance & $\begin{array}{l}\text { P2 Perspective Taking, Normative Consensus X P2 } \\
\text { Perspective Taking }\end{array}$ & 0.00 \\
\hline group & covariance & $\begin{array}{l}\text { P2 Perspective Taking, Distinctive Consensus X P2 } \\
\text { Perspective Taking }\end{array}$ & 0.00 \\
\hline group & covariance & $\begin{array}{l}\text { P2 Perspective Taking, P2 Perspective Taking X } \\
\text { Positivity Bias }\end{array}$ & 0.00 \\
\hline group & covariance & $\begin{array}{l}\text { Normative Consensus X P2 Perspective Taking, } \\
\text { Distinctive Consensus X P2 Perspective Taking }\end{array}$ & 0.00 \\
\hline group & covariance & $\begin{array}{l}\text { Normative Consensus X P2 Perspective Taking, P2 } \\
\text { Perspective Taking X Positivity Bias }\end{array}$ & -0.01 \\
\hline group & covariance & $\begin{array}{l}\text { Distinctive Consensus X P2 Perspective Taking, P2 } \\
\text { Perspective Taking X Positivity Bias }\end{array}$ & 0.00 \\
\hline
\end{tabular}


NETWORK APPROACH TO REPUTATIONS: SUPPLEMENT

\begin{tabular}{llll}
\hline source & type & term & Est. \\
\hline Residual & variance & Observation & 0.69 \\
\hline
\end{tabular}


NETWORK APPROACH TO REPUTATIONS: SUPPLEMENT

Table S33

Random Effects for P2 Fantasy Moderating Hearsay Accuracy (SAM)

\begin{tabular}{|c|c|c|c|}
\hline source & type & term & Est. \\
\hline triad:group & variance & Intercept & 0.00 \\
\hline triad:group & variance & Normative Accuracy & 0.05 \\
\hline triad:group & variance & Distinctive Accuracy & 0.01 \\
\hline triad:group & variance & P2 Fantasy & 0.00 \\
\hline triad:group & variance & Normative Accuracy X P2 Fantasy & 0.02 \\
\hline triad:group & variance & Distinctive Accuracy X P2 Fantasy & 0.00 \\
\hline triad:group & variance & P2 Fantasy X Positivity Bias & 0.32 \\
\hline triad:group & covariance & Intercept, Normative Accuracy & -0.01 \\
\hline triad:group & covariance & Intercept, Distinctive Accuracy & 0.00 \\
\hline triad:group & covariance & Intercept, P2 Fantasy & 0.00 \\
\hline triad:group & covariance & Intercept, Normative Accuracy X P2 Fantasy & 0.00 \\
\hline triad:group & covariance & Intercept, Distinctive Accuracy X P2 Fantasy & 0.00 \\
\hline triad:group & covariance & Intercept, P2 Fantasy X Positivity Bias & 0.00 \\
\hline triad:group & covariance & Normative Accuracy, Distinctive Accuracy & 0.01 \\
\hline triad:group & covariance & Normative Accuracy, P2 Fantasy & 0.01 \\
\hline triad:group & covariance & Normative Accuracy, Normative Accuracy X P2 Fantasy & 0.01 \\
\hline triad:group & covariance & Normative Accuracy, Distinctive Accuracy X P2 Fantasy & 0.00 \\
\hline triad:group & covariance & Normative Accuracy, P2 Fantasy X Positivity Bias & -0.01 \\
\hline triad:group & covariance & Distinctive Accuracy, P2 Fantasy & 0.00 \\
\hline triad:group & covariance & Distinctive Accuracy, Normative Accuracy X P2 Fantasy & 0.00 \\
\hline triad:group & covariance & Distinctive Accuracy, Distinctive Accuracy X P2 Fantasy & 0.00 \\
\hline triad:group & covariance & Distinctive Accuracy, P2 Fantasy X Positivity Bias & 0.00 \\
\hline
\end{tabular}




\begin{tabular}{|c|c|c|c|}
\hline source & type & term & Est. \\
\hline triad:group & covariance & P2 Fantasy, Normative Accuracy X P2 Fantasy & 0.01 \\
\hline triad:group & covariance & P2 Fantasy, Distinctive Accuracy X P2 Fantasy & 0.00 \\
\hline triad:group & covariance & P2 Fantasy, P2 Fantasy X Positivity Bias & -0.02 \\
\hline triad:group & covariance & $\begin{array}{l}\text { Normative Accuracy X P2 Fantasy, Distinctive Accuracy } \\
\text { X P2 Fantasy }\end{array}$ & 0.00 \\
\hline triad:group & covariance & $\begin{array}{l}\text { Normative Accuracy X P2 Fantasy, P2 Fantasy X } \\
\text { Positivity Bias }\end{array}$ & -0.08 \\
\hline triad:group & covariance & $\begin{array}{l}\text { Distinctive Accuracy X P2 Fantasy, P2 Fantasy X } \\
\text { Positivity Bias }\end{array}$ & -0.01 \\
\hline group & variance & Intercept & 0.00 \\
\hline group & variance & Normative Accuracy & 0.01 \\
\hline group & variance & Distinctive Accuracy & 0.00 \\
\hline group & variance & P2 Fantasy & 0.00 \\
\hline group & variance & Normative Accuracy X P2 Fantasy & 0.00 \\
\hline group & variance & Distinctive Accuracy X P2 Fantasy & 0.00 \\
\hline group & variance & P2 Fantasy X Positivity Bias & 0.04 \\
\hline group & covariance & Intercept, Normative Accuracy & 0.00 \\
\hline group & covariance & Intercept, Distinctive Accuracy & 0.00 \\
\hline group & covariance & Intercept, P2 Fantasy & 0.00 \\
\hline group & covariance & Intercept, Normative Accuracy X P2 Fantasy & 0.00 \\
\hline group & covariance & Intercept, Distinctive Accuracy X P2 Fantasy & 0.00 \\
\hline group & covariance & Intercept, P2 Fantasy X Positivity Bias & 0.00 \\
\hline group & covariance & Normative Accuracy, Distinctive Accuracy & 0.00 \\
\hline group & covariance & Normative Accuracy, P2 Fantasy & 0.00 \\
\hline group & covariance & Normative Accuracy, Normative Accuracy X P2 Fantasy & 0.00 \\
\hline
\end{tabular}


NETWORK APPROACH TO REPUTATIONS: SUPPLEMENT

\begin{tabular}{|c|c|c|c|}
\hline source & type & term & Est. \\
\hline group & covariance & Normative Accuracy, Distinctive Accuracy X P2 Fantasy & 0.00 \\
\hline group & covariance & Normative Accuracy, P2 Fantasy X Positivity Bias & 0.01 \\
\hline group & covariance & Distinctive Accuracy, P2 Fantasy & 0.00 \\
\hline group & covariance & Distinctive Accuracy, Normative Accuracy X P2 Fantasy & 0.00 \\
\hline group & covariance & Distinctive Accuracy, Distinctive Accuracy X P2 Fantasy & 0.00 \\
\hline group & covariance & Distinctive Accuracy, P2 Fantasy X Positivity Bias & 0.00 \\
\hline group & covariance & P2 Fantasy, Normative Accuracy X P2 Fantasy & 0.00 \\
\hline group & covariance & P2 Fantasy, Distinctive Accuracy X P2 Fantasy & 0.00 \\
\hline group & covariance & P2 Fantasy, P2 Fantasy X Positivity Bias & -0.01 \\
\hline group & covariance & $\begin{array}{l}\text { Normative Accuracy X P2 Fantasy, Distinctive Accuracy } \\
\text { X P2 Fantasy }\end{array}$ & 0.00 \\
\hline group & covariance & $\begin{array}{l}\text { Normative Accuracy X P2 Fantasy, P2 Fantasy X } \\
\text { Positivity Bias }\end{array}$ & 0.00 \\
\hline group & covariance & $\begin{array}{l}\text { Distinctive Accuracy X P2 Fantasy, P2 Fantasy X } \\
\text { Positivity Bias }\end{array}$ & 0.00 \\
\hline Residual & & & 0.75 \\
\hline
\end{tabular}


NETWORK APPROACH TO REPUTATIONS: SUPPLEMENT

Table S34

Random Effects for P2 Perspective Taking Moderating Hearsay Accuracy (SAM)

\begin{tabular}{|c|c|c|c|}
\hline source & type & term & Est. \\
\hline triad:group & variance & Intercept & 0.00 \\
\hline triad:group & variance & Normative Accuracy & 0.04 \\
\hline triad:group & variance & Distinctive Accuracy & 0.01 \\
\hline triad:group & variance & P2 Perspective Taking & 0.01 \\
\hline triad:group & variance & Normative Accuracy X P2 Perspective Taking & 0.05 \\
\hline triad:group & variance & Distinctive Accuracy X P2 Perspective Taking & 0.00 \\
\hline triad:group & variance & P2 Perspective Taking X Positivity Bias & 0.63 \\
\hline triad:group & covariance & Intercept, Normative Accuracy & -0.01 \\
\hline triad:group & covariance & Intercept, Distinctive Accuracy & 0.00 \\
\hline triad:group & covariance & Intercept, P2 Perspective Taking & 0.00 \\
\hline triad:group & covariance & Intercept, Normative Accuracy X P2 Perspective Taking & 0.00 \\
\hline triad:group & covariance & Intercept, Distinctive Accuracy X P2 Perspective Taking & 0.00 \\
\hline triad:group & covariance & Intercept, P2 Perspective Taking X Positivity Bias & 0.00 \\
\hline triad:group & covariance & Normative Accuracy, Distinctive Accuracy & 0.01 \\
\hline triad:group & covariance & Normative Accuracy, P2 Perspective Taking & 0.00 \\
\hline triad:group & covariance & $\begin{array}{l}\text { Normative Accuracy, Normative Accuracy X P2 } \\
\text { Perspective Taking }\end{array}$ & 0.00 \\
\hline triad:group & covariance & $\begin{array}{l}\text { Normative Accuracy, Distinctive Accuracy X P2 } \\
\text { Perspective Taking }\end{array}$ & 0.00 \\
\hline triad:group & covariance & $\begin{array}{l}\text { Normative Accuracy, P2 Perspective Taking X Positivity } \\
\text { Bias }\end{array}$ & 0.01 \\
\hline triad:group & covariance & Distinctive Accuracy, P2 Perspective Taking & 0.00 \\
\hline triad:group & covariance & $\begin{array}{l}\text { Distinctive Accuracy, Normative Accuracy X P2 } \\
\text { Perspective Taking }\end{array}$ & 0.00 \\
\hline
\end{tabular}




\begin{tabular}{|c|c|c|c|}
\hline source & type & term & Est. \\
\hline triad:group & covariance & $\begin{array}{l}\text { Distinctive Accuracy, Distinctive Accuracy X P2 } \\
\text { Perspective Taking }\end{array}$ & 0.00 \\
\hline triad:group & covariance & $\begin{array}{l}\text { Distinctive Accuracy, P2 Perspective Taking X Positivity } \\
\text { Bias }\end{array}$ & 0.01 \\
\hline triad:group & covariance & $\begin{array}{l}\text { P2 Perspective Taking, Normative Accuracy X P2 } \\
\text { Perspective Taking }\end{array}$ & 0.02 \\
\hline triad:group & covariance & $\begin{array}{l}\text { P2 Perspective Taking, Distinctive Accuracy X P2 } \\
\text { Perspective Taking }\end{array}$ & 0.00 \\
\hline triad:group & covariance & $\begin{array}{l}\text { P2 Perspective Taking, P2 Perspective Taking X } \\
\text { Positivity Bias }\end{array}$ & -0.07 \\
\hline triad:group & covariance & $\begin{array}{l}\text { Normative Accuracy X P2 Perspective Taking, } \\
\text { Distinctive Accuracy X P2 Perspective Taking }\end{array}$ & 0.01 \\
\hline triad:group & covariance & $\begin{array}{l}\text { Normative Accuracy X P2 Perspective Taking, P2 } \\
\text { Perspective Taking X Positivity Bias }\end{array}$ & -0.17 \\
\hline triad:group & covariance & $\begin{array}{l}\text { Distinctive Accuracy X P2 Perspective Taking, P2 } \\
\text { Perspective Taking X Positivity Bias }\end{array}$ & -0.03 \\
\hline group & variance & Intercept & 0.00 \\
\hline group & variance & Normative Accuracy & 0.01 \\
\hline group & variance & Distinctive Accuracy & 0.00 \\
\hline group & variance & P2 Perspective Taking & 0.00 \\
\hline group & variance & Normative Accuracy X P2 Perspective Taking & 0.00 \\
\hline group & variance & Distinctive Accuracy X P2 Perspective Taking & 0.00 \\
\hline group & variance & P2 Perspective Taking X Positivity Bias & 0.06 \\
\hline group & covariance & Intercept, Normative Accuracy & 0.00 \\
\hline group & covariance & Intercept, Distinctive Accuracy & 0.00 \\
\hline group & covariance & Intercept, P2 Perspective Taking & 0.00 \\
\hline group & covariance & Intercept, Normative Accuracy X P2 Perspective Taking & 0.00 \\
\hline
\end{tabular}




\begin{tabular}{|c|c|c|c|}
\hline source & type & term & Est. \\
\hline group & covariance & Intercept, Distinctive Accuracy X P2 Perspective Taking & 0.00 \\
\hline group & covariance & Intercept, P2 Perspective Taking X Positivity Bias & 0.01 \\
\hline group & covariance & Normative Accuracy, Distinctive Accuracy & 0.00 \\
\hline group & covariance & Normative Accuracy, P2 Perspective Taking & 0.00 \\
\hline group & covariance & $\begin{array}{l}\text { Normative Accuracy, Normative Accuracy X P2 } \\
\text { Perspective Taking }\end{array}$ & 0.00 \\
\hline group & covariance & $\begin{array}{l}\text { Normative Accuracy, Distinctive Accuracy X P2 } \\
\text { Perspective Taking }\end{array}$ & 0.00 \\
\hline group & covariance & $\begin{array}{l}\text { Normative Accuracy, P2 Perspective Taking X Positivity } \\
\text { Bias }\end{array}$ & -0.01 \\
\hline group & covariance & Distinctive Accuracy, P2 Perspective Taking & 0.00 \\
\hline group & covariance & $\begin{array}{l}\text { Distinctive Accuracy, Normative Accuracy X P2 } \\
\text { Perspective Taking }\end{array}$ & 0.00 \\
\hline group & covariance & $\begin{array}{l}\text { Distinctive Accuracy, Distinctive Accuracy X P2 } \\
\text { Perspective Taking }\end{array}$ & 0.00 \\
\hline group & covariance & $\begin{array}{l}\text { Distinctive Accuracy, P2 Perspective Taking X Positivity } \\
\text { Bias }\end{array}$ & 0.00 \\
\hline group & covariance & $\begin{array}{l}\text { P2 Perspective Taking, Normative Accuracy X P2 } \\
\text { Perspective Taking }\end{array}$ & 0.00 \\
\hline group & covariance & $\begin{array}{l}\text { P2 Perspective Taking, Distinctive Accuracy X P2 } \\
\text { Perspective Taking }\end{array}$ & 0.00 \\
\hline group & covariance & $\begin{array}{l}\text { P2 Perspective Taking, P2 Perspective Taking X } \\
\text { Positivity Bias }\end{array}$ & 0.00 \\
\hline group & covariance & $\begin{array}{l}\text { Normative Accuracy X P2 Perspective Taking, } \\
\text { Distinctive Accuracy X P2 Perspective Taking }\end{array}$ & 0.00 \\
\hline group & covariance & $\begin{array}{l}\text { Normative Accuracy X P2 Perspective Taking, P2 } \\
\text { Perspective Taking X Positivity Bias }\end{array}$ & 0.01 \\
\hline group & covariance & $\begin{array}{l}\text { Distinctive Accuracy X P2 Perspective Taking, P2 } \\
\text { Perspective Taking X Positivity Bias }\end{array}$ & 0.01 \\
\hline
\end{tabular}


NETWORK APPROACH TO REPUTATIONS: SUPPLEMENT

\begin{tabular}{llrr}
\hline source & type & term & Est. \\
\hline Residual & & 0.75 \\
\hline
\end{tabular}


NETWORK APPROACH TO REPUTATIONS: SUPPLEMENT

Table S35

Model Comparisons for Testing Condition Differences for Extraversion

\begin{tabular}{lcccc}
\hline Equality Constraint & $\Delta_{\text {AIC }}$ & $\Delta_{\text {BIC }}$ & $\chi_{\mathrm{d}^{2}}$ & $p$ \\
\hline Hearsay Consensus & -0.65 & -3.29 & 1.35 & .244 \\
Hearsay Accuracy & -1.88 & -4.52 & 0.12 & .727 \\
Direct Accuracy & 0.22 & -2.42 & 2.22 & .136
\end{tabular}

Note. $\Delta_{\text {AIC }}$ and $\Delta_{\text {BIC }}$ are the change in AIC and BIC (respectively) resulting from the constraint; negative values indicate that the constraint leads to better fit, and positive values indicate that the constraint leads to worse fit. $\chi_{\mathrm{d}}{ }^{2}$ is the difference in $\chi^{2}$ between the model with and without constraints. Each model is compared to the baseline model (i.e., the model with no crosscondition equality constraints). Equality constraints were imposed across the Status and Control conditions for Extraversion. The difference in $d f_{\text {model }}$ for each comparison is 1 . 
NETWORK APPROACH TO REPUTATIONS: SUPPLEMENT

Table S36

Model Comparisons for Testing Condition Differences for Agreeableness

\begin{tabular}{lcccc}
\hline Equality Constraint & $\Delta_{\text {AIC }}$ & $\Delta_{\text {BIC }}$ & $\chi_{\mathrm{d}}{ }^{2}$ & $p$ \\
\hline Hearsay Consensus & -1.89 & -4.54 & 0.11 & .741 \\
Hearsay Accuracy & -1.06 & -3.71 & 0.94 & .333 \\
Direct Accuracy & -1.99 & -4.64 & 0.01 & .930 \\
\hline
\end{tabular}

Note. $\Delta_{\text {AIC }}$ and $\Delta_{\text {BIC }}$ are the change in AIC and BIC (respectively) resulting from the constraint; negative values indicate that the constraint leads to better fit, and positive values indicate that the constraint leads to worse fit. $\chi_{\mathrm{d}}{ }^{2}$ is the difference in $\chi^{2}$ between the model with and without constraints. Each model is compared to the baseline model (i.e., the model with no crosscondition equality constraints). Equality constraints were imposed across the Affiliation and Control conditions for Agreeableness. The difference in $d f_{\text {model }}$ for each comparison is 1 . 
NETWORK APPROACH TO REPUTATIONS: SUPPLEMENT

Table S37

Model Comparisons Testing for Between Study Differences in Full Variance-Covariance and Mean Structure

\begin{tabular}{lrrrl}
\hline & \multicolumn{1}{c}{$\Delta_{\text {AIC }}$} & $\Delta_{\text {BIC }}$ & \multicolumn{1}{c}{$\chi_{\mathrm{d}}^{2}$} & $p$ \\
\hline agreeableness & -15.82 & -63.47 & 14.18 & .512 \\
conscientiousness & -5.04 & -52.68 & 24.96 & .050 \\
honesty-propriety & -11.61 & -59.25 & 18.39 & .243 \\
neuroticism & -8.08 & -55.72 & 21.92 & .110 \\
extraversion & -20.50 & -68.14 & 9.50 & .850 \\
openness & -14.31 & -61.95 & 15.69 & .403 \\
\hline
\end{tabular}

Note. The $d f_{\text {model }}$ is 24 for the model allowing studies to differ and 39 for the model pooling across studies; the $d f$ for the model comparison is therefore $15 . \Delta_{\text {AIC }}$ and $\Delta_{\text {BIC }}=$ the difference in AIC and BIC (respectively) between the model allowing studies to differ and the model pooling across studies; negative values indicate better fit for the pooled (integrated) model. 


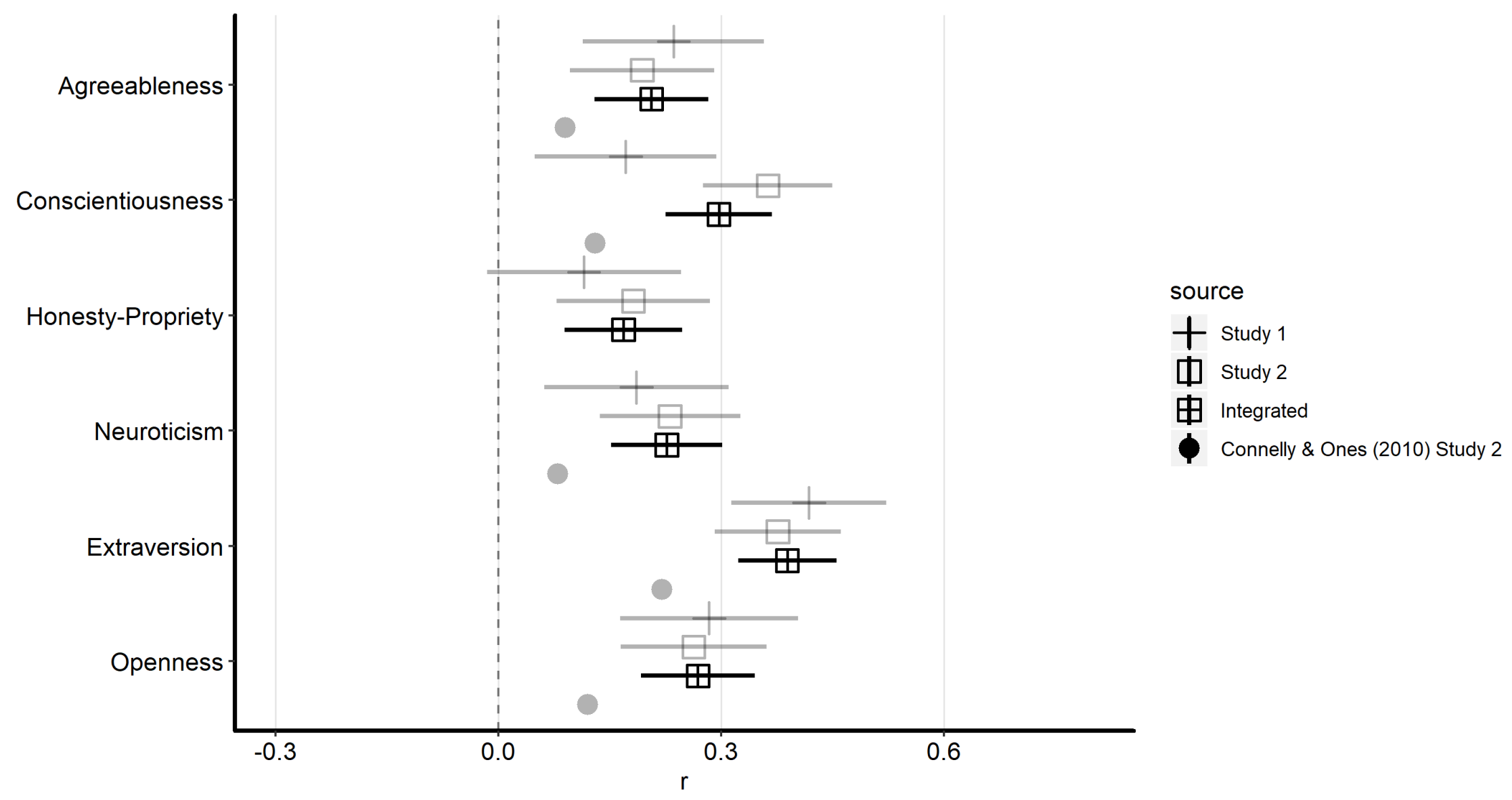

Figure S1. Study 1, Study 2, and integrated estimates of direct accuracy for the Big Six. Study 1 (box) estimates are based just on the Study 1 sample; Study 2 (cross) estimates are based just on the Study 2 sample (collapsing across condition); integrated estimates (crossed-box) are based on data from both Studies. Circles represent meta-analytic estimates of self-other agreement among previously unacquainted participants, obtained from Study 2 of Connelly \& Ones's (2010) meta-analysis on consensus and self-other agreement. 


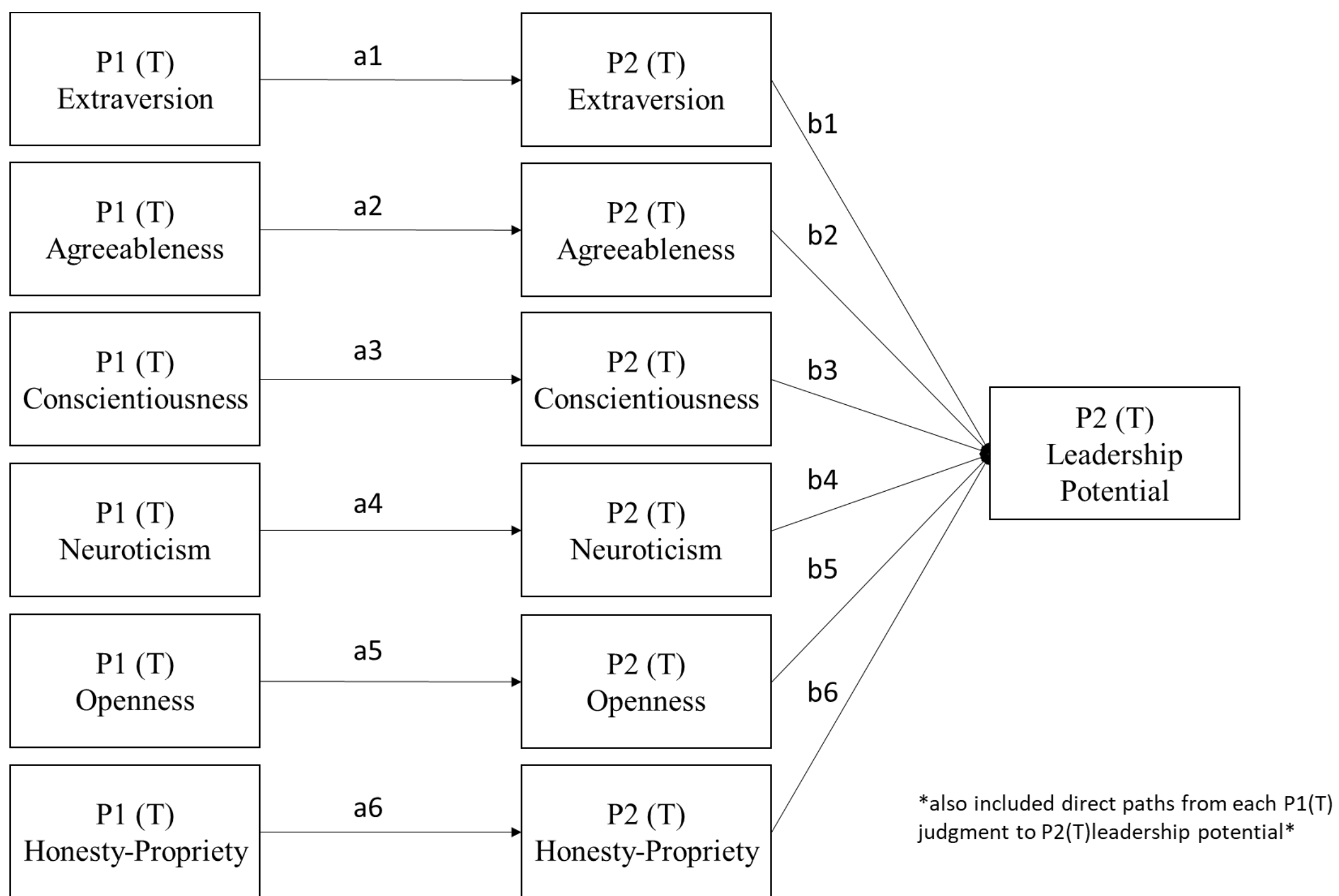

Figure S2. Path model predicting hearsay reputations of leadership potential from hearsay and direct reputations of all Big Six. The model also included direct effects from each P1(T) judgement to P2(T) leadership potential and tested each indirect effect (not depicted). 


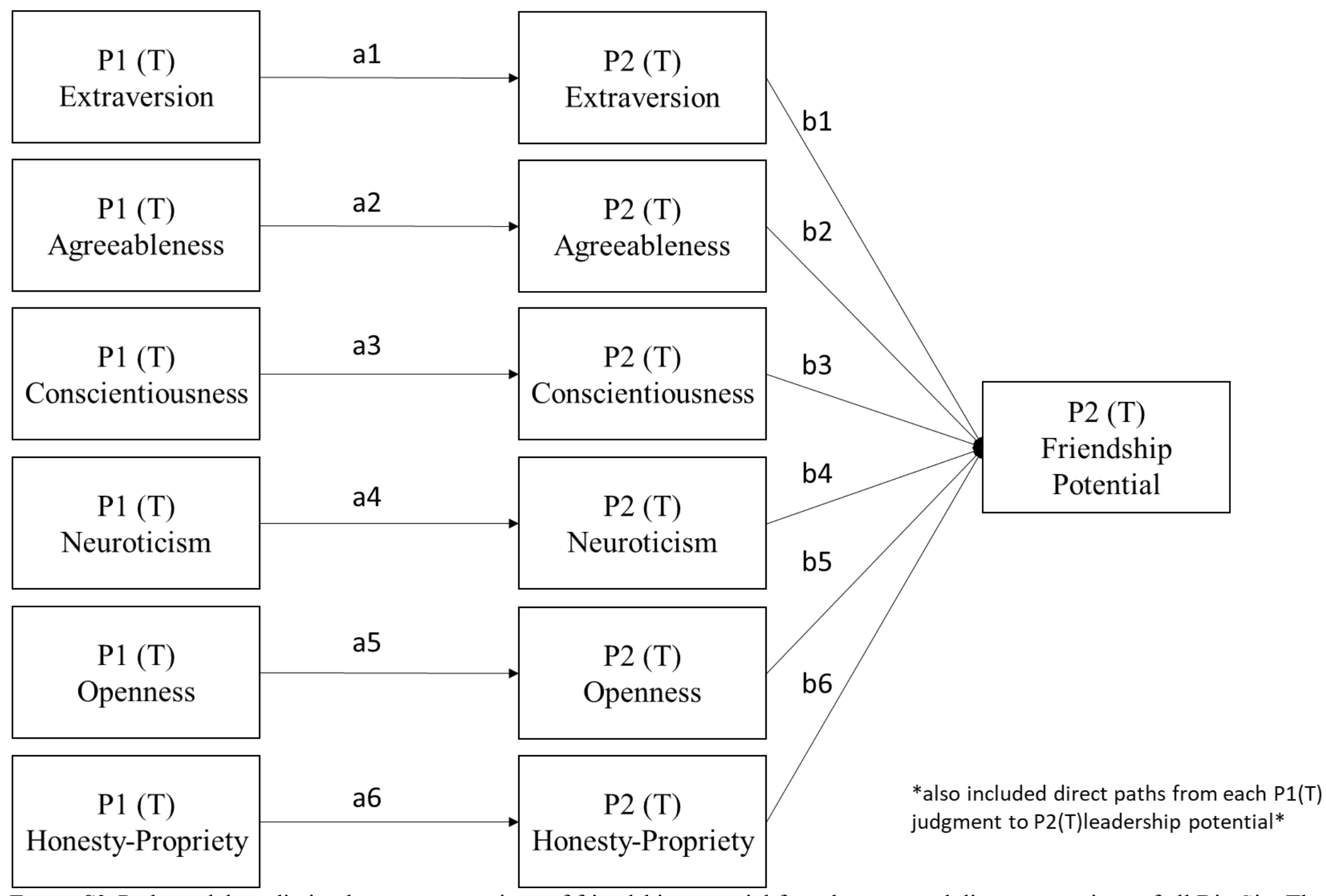

Figure S3. Path model predicting hearsay reputations of friendship potential from hearsay and direct reputations of all Big Six. The model also included direct effects from each P1(T) judgement to P2(T) leadership potential and tested each indirect effect (not depicted). 